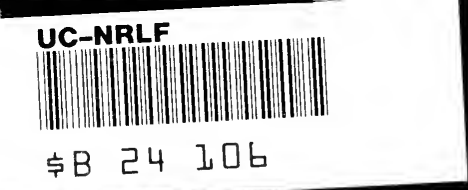

ifis

Wtation

$=25,7,25,04=$

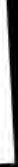

it 


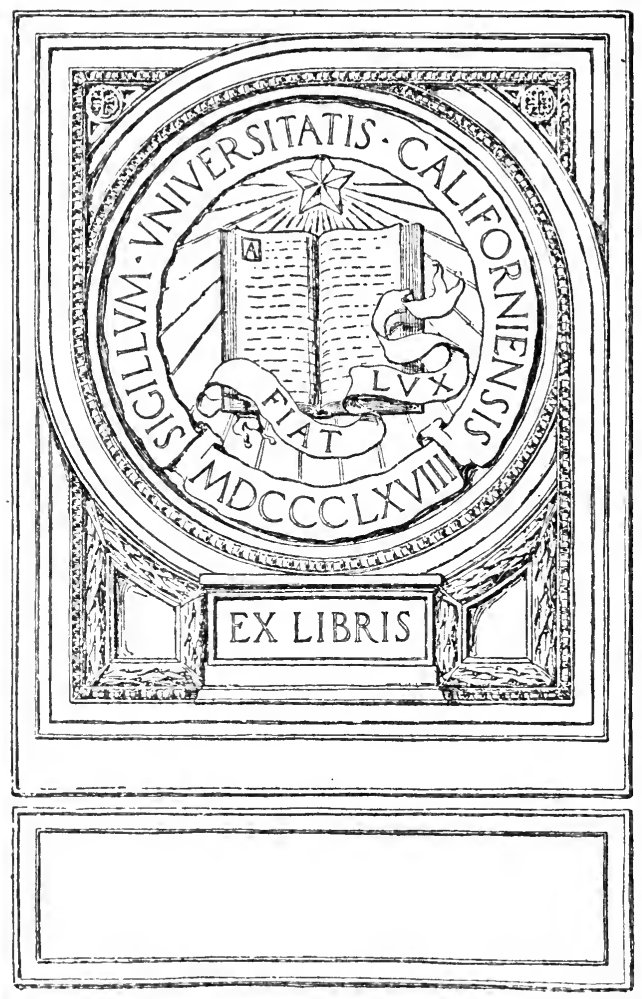




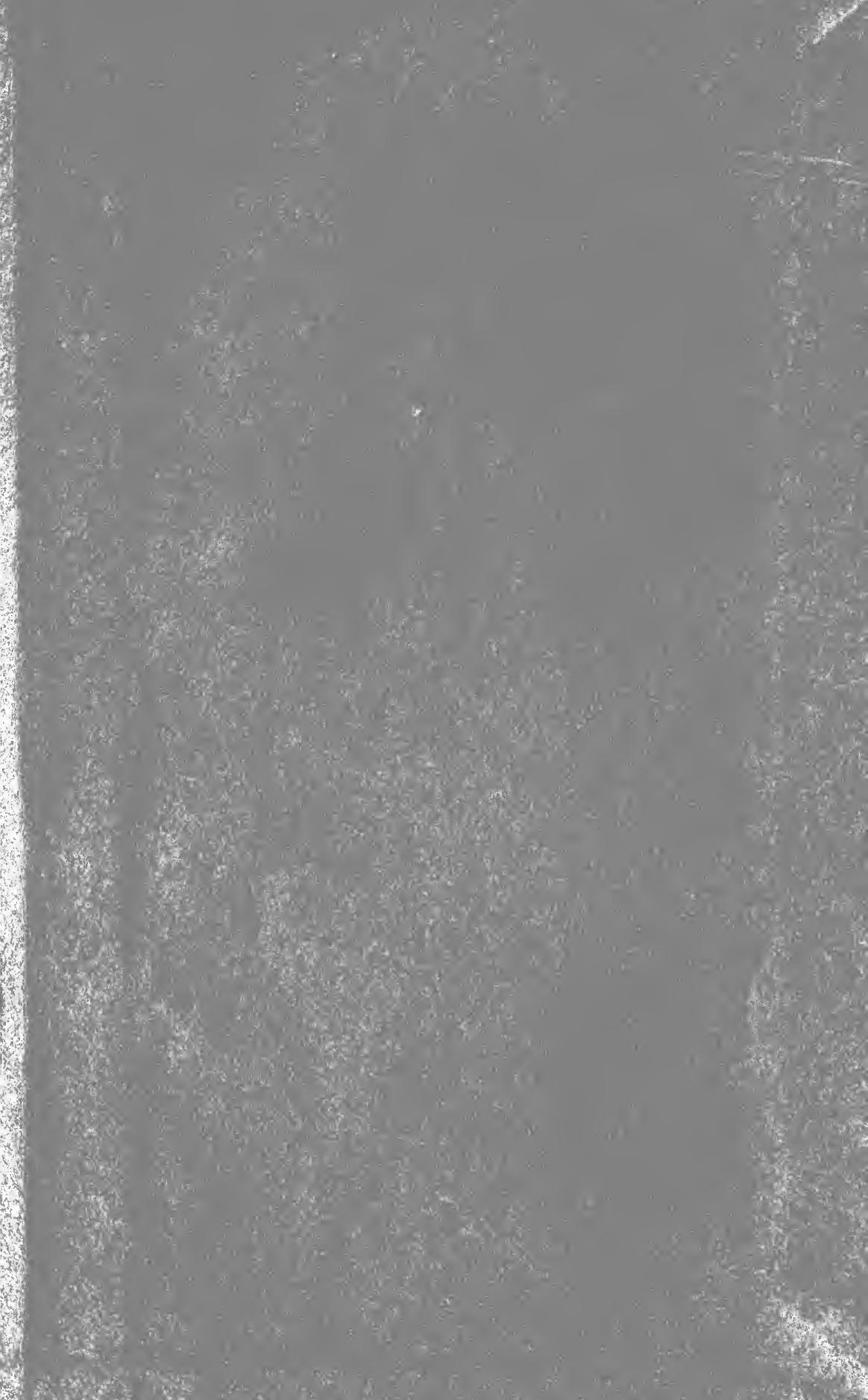



PRACTICAL MEASUREMENTS IN

RADIO-ACTIVITY 



\section{PRACTICAL MEASUREMENTS}

\section{IN RADIO-ACTIVITY.}

BY

W. MAKOWER, M.A., D.Sc.

LECTURER AND DEMONSTRATOR IN PHYSICS IN THE UNIVERSITY OF MANCHESTER

AND

H. GEIGER, Рн.D.

LECTURER IN PHYSICS IN THE UNIVERSITY OF MANCHESTER

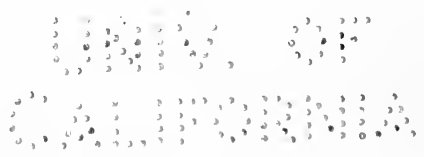

LONGMANS, GREEN, AND CO.

39 PATERNOSTER ROW, LONDON NEW YORK, BOMBAY, AND CALCUTTA

1912

All rights reserved 
QC 75

MB

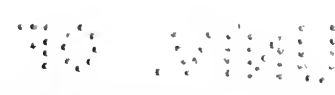

$\therefore$ 무 


\section{PREFACE.}

Although researches into the nature of radio-activity have proved one of the most fruitful means of investigating atomic phenomena, a study of the methods employed for making radio-active measurements has not yet come to be regarded as an essential part of a course of practical physics or chemistry. This is no doubt to some extent due to a belief that a practical course must necessarily involve the use of large and costly amounts of radio-active material. This is, however, not the case, for it is possible to illustrate most of the principles of radio-activity by experiments made with simple apparatus using quite small quantities of radio-active material. To show how this can be done has been the main object of describing a number of experiments, many of which have been designed by Professor Rutherford as part of the course of practical work in the Honours School of Physics in the Manchester University and as an introductory course for students intending to undertake original investigations in radio-activity. It is hoped that this work may do something towards giving the subject a more prominent position in laboratory courses, and thus teach students the technique of one of the most fascinating branches of modern science.

Although the book is primarily intended as a laboratory course in radio-activity, an attempt has been made to meet the requirements of those engaged in original investigations. On that account tables of radio-active constants and of the decay of different substances have been given; moreover, some portions of the subject have been dealt with in greater detail than would otherwise be necessary. Thus, in Chapter 
VII., the methods of making accurate standard measurements are given rather fully, and in Chapter VIII. are given methods of separating radio-active products from others with which they are usually associated. For much of the information given in that chapter, the authors are indebted to Professor B. B. Boltwood. Since the theory of the quadrant electrometer given in most text-books is inadequate, some space is devoted to the exposition of the theory of the instrument recently given by Professor Beattie; for this treatment, though only approximate, corresponds with the conditions under which the instrument is generally used and makes clear the physical factors controlling its behaviour.

The book is intended to be as far as possible self-contained, and the theory of each experiment is usually briefly stated. Elaborate discussions of theoretical points are avoided, and it is assumed that the reader has an elementary knowledge of radio-activity. In many cases references are made to the new edition of Professor Rutherford's treatise on Radioactivity, where full theoretical discussions will be found.

The authors have endeavoured to avoid errors and inaccuracies. If this object has been attained, they feel that it is in no small measure due to the assistance given them by their friends and colleagues who have supplied them with suggestions and data on many subjects. In particular, they would express their thanks to Mr. H. G. J. Moseley for reading and criticising the manuscript, and to Dr. S. Russ and Mr. E. Marsden for correcting the proof sheets. They are also indebted for a number of suggestions to Professor Rutherford who, by instituting a course of practical measurements in radio-activity in this laboratory, has inspired this work.

W. M.

H. G.

Physical Laboratory,

The University,

Manchester, September, 1912. 


\title{
CONTENTS.
}

\author{
CHAPTER I.
}

THE QUADRANT ELECTROMETER AND INSTRUMENTS USED IN CONNECTION WITH IT.

SECT.

1. Construction and Adjustment of the Quadrant Electrometer - - 1

2. Theory of the Quadrant Electrometer - _ _ _ _ _ - 3

3. Variation of the Sensitiveness of an Electrometer with the Poten-

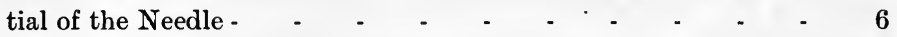

4. Calibration of the Scale of a Quadrant Electrometer - - - $\quad 6$

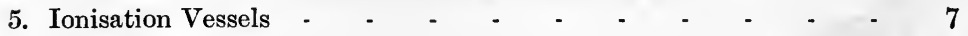

6. The Earthing Key - $\quad$ - $\quad$ - $\quad$ - $\quad$ - $\quad$ - $\quad$ - $\quad$ - $\quad$ - $\quad$ - 9

7. Measurements of Ionisation Currents - - - - - - $\quad$ - 10

8. Natural Leak of Electrometer and Insulation Tests $\quad$ - $\quad$ - $\quad$ - 12

9. Constant Deflection Method of Measuring Ionisation Currents - 13

10. Zero Method of Measuring Ionisation Currents - - - $\quad$ - 15

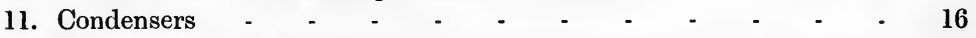

12. Measurement of Capacities of Condensers by Multiple Discharge Method - - $\quad$ - $\quad$ - $\quad$ - $\quad$ - $\quad$ - $\quad$ - 17

13. Measurement of Capacities by Ionisation Currents - $\quad$ - $\quad$ - $\quad 18$

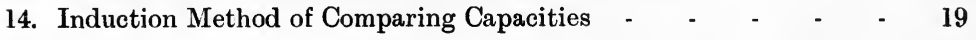

\section{CHAPTER II.}

\section{ELECTROSCOPES.}

15. Alpha-ray Electroscopes - - $\quad$ - $\quad$ - $\quad$ - $\quad$ - $\quad$ - $\quad$ - 21

16. Beta and Gamma-ray Electroscopes $\quad$ - $\quad$ - $\quad$ - $\quad$ - $\quad$ - 22

17. Methods of making Measurements with Electroscopes - - - $\quad 23$

18. Calibration of the Scale of an Electroscope - - - - - - 24

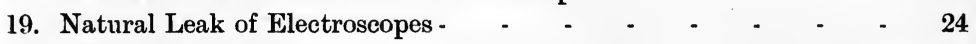

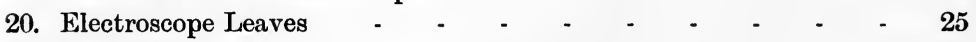

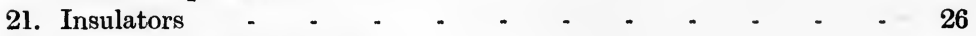

22. Emanation Electroseopes - $\quad$ - $\quad$ - $\quad$ - $\quad$ - $\quad$ - $\quad$ - $\quad$ - $\quad$ - 28

23. The Tilted Electroscope - $\quad$ - $\quad$ - $\quad$ - $\quad$ - $\quad$ - $\quad$ - $\quad$ - $\quad$ - 29

24. Quartz-fibre Electroscope - - - - - $\quad$ - $\quad$ - $\quad 30$ 


\section{CHAPTER III.}

SECT.

\section{THE IONISATION OF GASES.}

25. The Ionisation of Gases by Radiations - _ _ - _ 32

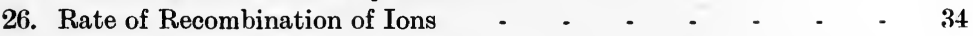

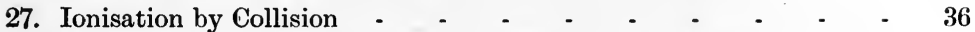

28. Measurement of the Ratio of the Charge to the Mass and of the

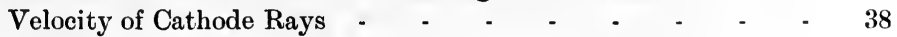

\section{CHAPTER IV.}

\section{THE ALPHA RAYS.}

29. Ionisation produced by different Areas of Uranium Oxide $\quad$ - $\quad$ - $\quad 42$

30. Ionisation produced by different Thicknesses of Uranium Oxide - $\quad 42$

31. The Ranges of $a$ Particles and their Absorption by Matter - - 44

32. The Variation of the Ionisation produced by an a Particle along its Path

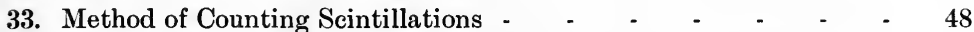

34. Determination of the Range of $a$ Particles by Scintillations - $\quad-\quad 50$

35. The sudden Stoppage of a Particles at the End of their Range - $\quad 50$

36. The Stopping Power of Metal Foils - _ - _ - - $\quad$ - $\quad$ - 52

37. The Laws of Probability as applied to the Emission of $a$ Particles - $\quad 53$

\section{CHAPTER V.}

\section{THE BETA AND GAMMA RAYS.}

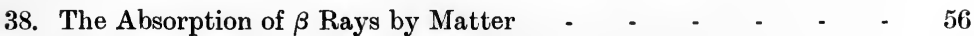

39. The $\beta$ Radiation from different Thicknesses of Uranium - $\quad$ - $\quad$ - 60

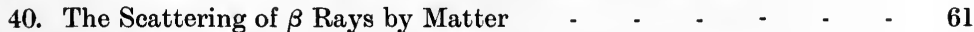

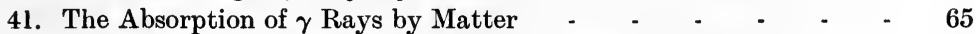

42. Comparison of Absorptions of $a, \beta$ and $\gamma$ Rays by Matter $\quad-\quad$ - $\quad 67$

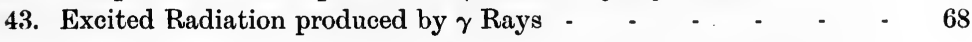

\section{CHAPTER VI.}

\section{THE ACTIVE DEPOSITS AND RADIO-ACTIVE RECOIL.}

44. The Distribution of the Active Deposits in an Electric Field and

the Method of concentrating them on Surfaces - - - - 71

45. Activity acquired by a Wire in electric Fields of varying Intensity 74

46. Radio-active Recoil - $\quad$ - $\quad$ - $\quad$ - $\quad$ - $\quad$ - $\quad$ - $\quad$ - $\quad$ - $\quad$ - 75

47. The Efficiency of Recoil - _ _ - _ - _ - - $\quad$ - 78 


\section{CHAPTER VII.}

skct.

\section{RADIO-ACTIVE TRANSFORMATIONS.}

48. Exponential Decay of Radio-active Substances - - - - $\quad-80$

49. Decay of Thorium and Actinium Active Deposits (short exposure) - $\quad 83$

50. Decay of Thorium and Actinium Active Deposits (long exposure) - 88

51. Decay of the Active Deposit of Radium - - - - - - $\quad 90$

52. Decay and Recovery of Radio-active Bodies - _ _ _ _ $\quad$ - 93

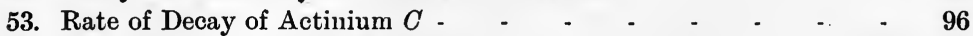

54. Comparison of the Rates of Decay of the Emanations - - $\quad$ - $\quad$ - $\quad 97$

55. Rate of Decay of Actinium Emanation - - - - - - $\quad$ - 98

56. Rate of Decay of very short-lived Products - _ _ _ $\quad$ - 100

57. Diffusion of Thorium and Actinium Emanations - - - - $\quad$ - 101

\section{CHAPTER VIII.}

\section{STANDARD MEASUREMENTS.}

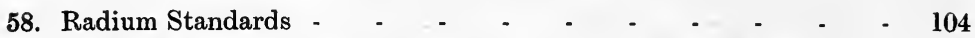

59. Comparison of Quantities of Radium by a $\gamma$-ray Electroscope - _ - 106

60. Balance Method of standardising Radium _ _ _ - - $\quad$ - 108

61. Quantitative Estimation of Radium Emanation - _ _ $\quad 110$

62. Determination of small Quantities of Radium (Emanation method) 113

63. Activity of Minerals and Mineral Waters - - - - - $\quad 116$

64. Standardisation by the Method of Scintillations _ - - - $\quad 117$

\section{CHAPTER IX.}

\section{THE SEPARATION OF RADIO-ACTIVE SUBSTANCES.}

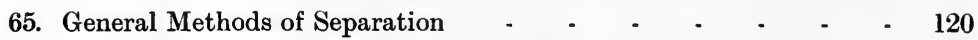

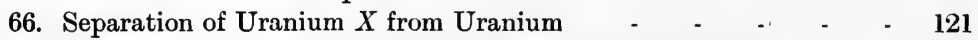

67. Separation of Radium $C$ from the Active Deposit of Radium - $\quad-\quad 122$

68. Separation of Radium $D$ and Radium $E-\quad-\quad-\quad-\quad-\quad-\quad 122$

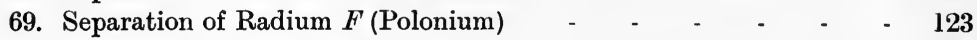

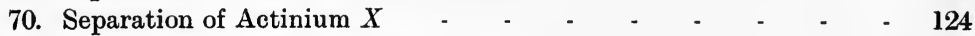

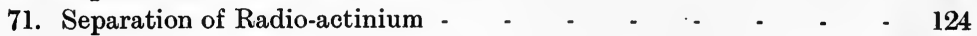

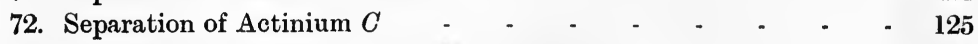

73. Separation of Thorium $X$ and Mesothorium - - - - - - 125

74. Separation of Thorium $B$ and Thorium $C \quad-\quad-\quad-\quad-\quad-127$

75. Separation of Emanation from Radium Solutions - _ _ - 127

Appendix I. Tables of Radio-active Constants - - - - $\quad 134$

Appendix II. Tables of 1)eay of Radio-active Substances - - 138

Appendix III. Notes on Radio-active Materials and Measuring Instruments - $\quad-\quad \cdot \quad-\quad+\quad-\quad$ - 149

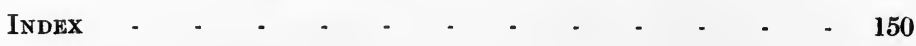





\title{
PRACTICAL MEASUREMENTS IN RADIO-ACTIVITY.
}

\author{
CHAPTER I. .
}

THE QUADRANT ELECTROMETER AND INSTRUMENTS USED IN CONNECTION WITH IT.

\section{$\$ 1$. Construction and Adjustment of the Quadrant Electro- meter.}

Electric currents through gases are usually very small, and except in cases of intense ionisation it is impossible to measure them with a galvanometer. For this reason special instruments have to be employed, and one of the most suitable is the quadrant electrometer. The original and earlier forms of the instrument are difficult to adjust, but the type more recently devised by Dolezalek can conveniently be used as an instrument of precision. The instrument which is shown in figure 1 consists essentially of the needle which is suspended by a thin phosphor-bronze strip so as to swing freely in four hollow quadrant-shaped metal boxes $A$ supported on amber insulating pillars $P$, the diagonally opposite pairs of quadrants being in metallic connection. The chief modifications introduced in the instru-

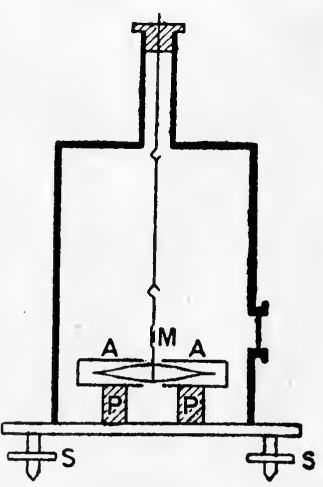

FIG. 1. ment consist in the smallness of the quadrants and the lightness of the needle, which is made of paper coated with a thin film of silver instead of being made of metal. The shape of the needle will be seen from the drawings in plan R.-A. 
and section given in figure 2. The needle can be maintained at a constant potential by keeping it permanently connected to a battery. The position of the needle is read by means of a beam of light reflected on to a scale from a mirror $M$ attached to the needle.

The electrometer is mounted on a steady table and adjusted in the following way. The two pairs of quadrants, the needle and the case of the instrument are connected to earth. The needle is then brought as nearly as possible into a position symmetrical with respect to the quadrants, as shown in figure 2. It is then connected to one terminal of a battery
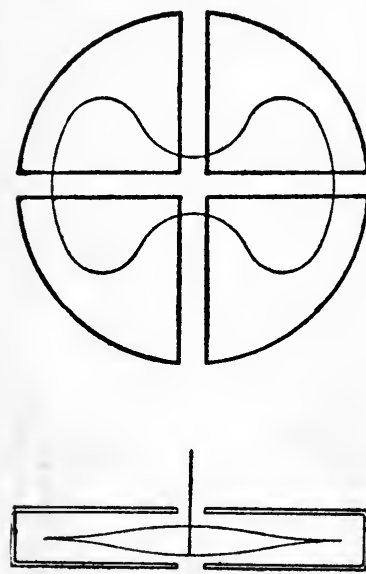

Fı.. 2. giving about 100 volts, the other terminal being connected to earth. A water resistance is introduced between the needle and the battery terminal to which it is connected, to prevent the possibility of a short circuit if the needle should accidentally touch the quadrants. On charging, the zero position of the needle is in general displaced. The needle is brought back to zero by adjusting the levelling screws $S$ and is then discharged; these operations are repeated several times until there is no appreciable change in the zero position on charging and discharging the needle which then hangs symmetrically between the quadrants. A further test of the accuracy of the adjustment should be made as described in $\$ 4$.

When very great sensitiveness is required a quartz fibre is used to suspend the needle instead of the phosphor-bronze strip, which cannot be obtained sufficiently fine. The quartz fibre can be rendered conducting by silvering or platinising, ${ }^{*}$ or by immersion in a solution of calcium chloride. In a dry climate, however, the latter method is unsatisfactory. If the quartz fibre is not made conducting the needle has to be

* Bestelmeyer, Zeitschr. für Instrumentenkunde, 25. 339, 1905. 
charged by momentary contact with one terminal of a battery. This method is, however, avoided whenever possible owing to the troublesome corrections which have to be applied on account of the change of sensitiveness of the instrument as the charge leaks away (\$3).

\section{$\$ 2$. Theory of the Quadrant Electrometer.}

According to the elementary theory of the quadrant electrometer usually given, the deflection $\theta$ suffered by the needle, the potential of which is $V$, is

$$
\theta=A\left(v_{1}-v_{2}\right)\left(V-\frac{v_{1}+v_{2}}{2}\right)
$$

where $A$ is a constant and $v_{1}$ and $v_{2}$ are the potentials of the opposite pairs of quadrants. It will thus be seen that the deflection should be proportional to the difference of potential of the opposite quadrants and to the potential $V$ of the needle, provided that this quantity is large compared with $v_{1}$ and $v_{2}$. When using the instrument for radio-active measurements in the way described in $\$ 7$, this condition holds, but it is found that the sensibility of the instrument is not proportional to the potential of the needle except within narrow limits. In fact the sensitiveness increases to a maximum as the potential of the needle is raised and then falls off for greater potentials. The theory has been amplified by various authors in order to explain this behaviour of the instrument, and the conclusions arrived at have recently been summarised and the theory given in a simple form by Beattie.* It is pointed out by Beattie that on deflecting the needle through a small angle $\theta$, there will be brought into play, due to the torsion in the suspension, a mechanical restoring couple given by $k_{1} \theta$, where $k_{1}$ is an instrumental constant. In addition to this couple a second one arises, due to electrical causes, even if the potentials of the opposite quadrants remain the same; for by the motion of the needle the lines of force between the needle and the quadrants are distorted in such a way as to introduce forces tending usually to restore the needle to its original position.

${ }^{*}$ R. Beattie, Electrician, 65. 729, 1910, and 69. 233, 1912. 
This couple will be called the distortional electrostatic control, and is said to be positive when it acts in the same direction as the mechanical control. If, however, as sometimes occurs, the needle is tilted relatively to the quadrants, it may happen that the distortional and mechanical controls oppose each other, when the former is said to be negative. The distortional control is proportional to $V^{2}$ and to the angle of deflection $\theta$, and may be written $k_{2} V^{2} \theta$, where $k_{2}$ is a constant.

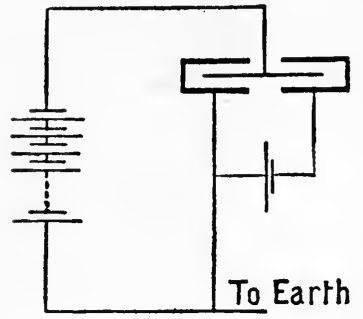

FIG. 3.

If the two opposite pairs of quadrants are permanently connected to the two poles of a battery as shown in figure 3 , the mechanical and distortional controls will be the only restoring couples brought into play, and when the needle has come to rest, their sum must be equal to the deflecting couple which is given by $k_{3} V\left(v_{1}-v_{2}\right)$. Hence, considering the distortional control as positive, we obtain the equation

$$
\begin{gathered}
k_{1} \theta+k_{2} V^{2} \theta=k_{3} V\left(v_{1}-v_{2}\right), \\
\theta=\frac{k_{3} V\left(v_{1}-v_{2}\right)}{k_{1}+k_{2} V^{2}} . \ldots .
\end{gathered}
$$

whence

Thus the sensibility of the instrument is proportional to

$$
\frac{k_{3} V}{k_{1}+k_{2} V^{2}}
$$

This quantity reaches a maximum when $V=\sqrt{\frac{k_{1}}{k_{2}}}$, i.e. when the mechanical and distortional controls are equal. If the distortional control is negative, the sensibility continually increases as the potential of the needle rises and the instrument ultimately becomes unstable.

If, as is usually the case in radio-active measurements, the two pairs of quadrants are not directly connected to a battery but are connected with an ionisation vessel $C$ (figure 4), a third controlling couple will be brought into play. This is due to a difference of potential set up between the insulated and earth-connected quadrants on account of 
a charge induced on the insulated quadrants by the motion of the charged needle. This couple is known as the inductional electrostatic control, and will resist the deflection of the needle. The charge induced on the insulated quadrants will be proportional to $V \theta$, and therefore the difference of potential set up between the quadrants will be proportional to $\frac{V \theta}{C}$, where $C$ is the total capacity of the electrometer and the ionisation vessel connected to it. Since this potential difference is always small compared with $V$, the resulting couple may be written $\frac{k_{4} V^{2} \theta}{C}$. If $q$ is the charge communicated to the insulated quadrants through the ionisation

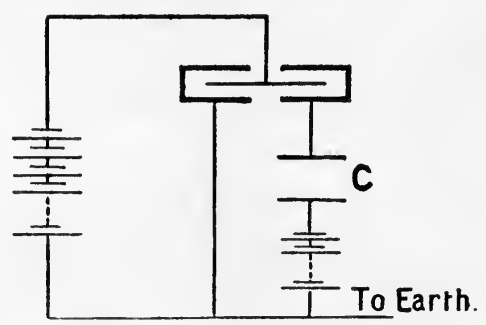

FIG. 4. vessel, the potential set up between the quadrants is $q / C$, and the corresponding deflecting couple on the needle is $\frac{k_{3} V q}{C}$. Hence, equating the deflecting and controlling couples, we have the relation

$$
k_{1} \theta+k_{2} V^{2} \theta+{ }^{k_{4}} V^{2} \theta=k_{3} \frac{V q}{C},
$$

whence

$$
\theta=\frac{k_{3} V}{k_{1}+k_{2} V^{2}+k_{4} C} \cdot \frac{q}{V^{2}} .
$$

The expression may be written in the form

$$
\theta=\frac{k_{3} V}{k_{1}} \cdot \frac{q}{C+\frac{k_{2} V^{2} C}{k_{1}}+\frac{k_{4} V^{2}}{k_{1}}}
$$

But if there is no restoring couple other than the mechanical control, the deflection is given by

$$
\theta=\frac{k_{3} V}{k_{1}} \cdot \frac{q}{C}
$$

Hence on account of the electrical control, the capacity of the electrometer appears to be altered from $C$ to $C+\frac{k_{2} V^{2} C}{k_{1}}+\frac{k_{4} V^{2}}{k_{1}}$, 
when the needle is deflected. This effect was first pointed out by J. J. Thomson; * but it is perhaps simpler to regard the capacity of the instrument as constant and the controlling couple changed in the manner expressed by equation (6).

\section{\$ 3. Variation of the Sensitiveness of an Electrometer with the Potential of the Needle.}

The theory of the variation of the sensitiveness of an electrometer with the potential of the needle has been discussed in $\S 2$. It follows from equations (4) and (6) that the variation of the sensitiveness of the instrument is different when used to measure differences of potential as shown in figure 3 , and when used to measure ionisation currents as shown in figure 4 . The variation of sensitiveness in the first case can be tested by charging the electrometer needle to different potentials varying up to several hundred volts, and observing the sensitiveness when a fixed potential difference is applied between the quadrants. In the second case, the sensitiveness can be tested by allowing a constant current to flow into the quadrants for a fixed time with the needle at different potentials. In both cases it will be found that the sensitiveness of the electrometer at first rises proportionally to the potential communicated to the needle. As the potential is increased, the sensitiveness rises less rapidly and ultimately reaches a maximum, after which it decreases as the potential of the needle is still further raised. The shapes of the curves will, however, be somewhat different in the two cases, and the maxima will in general occur for different voltages.

It is usually desirable to use the electrometer at maximum sensitiveness in order that small changes in the potential of the needle may be without sensible effect on the readings of the instrument.

\$4. Calibration of the Scale of a Quadrant Electrometer.

When an electrometer has been properly adjusted the deflection produced is proportional to the difference of

* J. J. Thomson, Phil. Mag. 46. 526, 1898. 
potential between the two pairs of quadrants, provided that this difference is small compared with the potential of the needle. Moreover, the deflections obtained with potentials of the same magnitude but of opposite signs are symmetrical with respect to the zero position.

To test the uniformity of the scale, the instrument can be calibrated in the following way. A current is sent through the resistance $A B$ from the storage cell $C$, one terminal of which is earthed (fig. 5). The resistance $A B$ should be

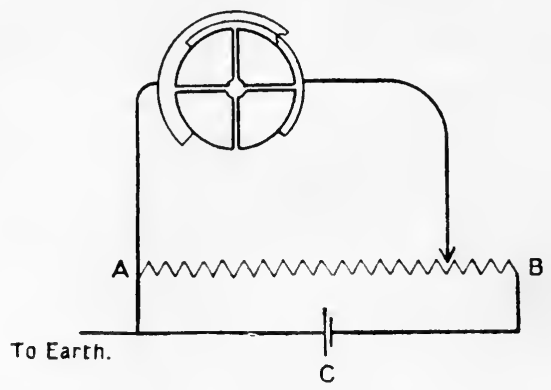

FIG. 5 .

large, and a subdivided megohm is suitable for the purpose. The insulated quadrants of the electrometer are connected to different points of the resistance so as to be brought to different potentials while the other quadrants remain earthed. A curve should be plotted showing the relation between the potential applied and the deflection of the electrometer needle. The relation is approximately linear. With an electrometer as usually set up a deflection of the order of 200 millimetres per volt is obtained on a scale placed a metre from the electrometer; but much greater sensitiveness can be attained with specially fine suspensions (§1).

\section{\$5. Ionisation Vessels.}

The most convenient form of ionisation vessel is that shown in figure 6 , consisting of a cylindrical vessel of about 300 c.c. capacity, along the axis of which passes a metal $\operatorname{rod} A$ which can be connected to the electrometer. The vessel is connected to one pole bf a battery of about 100 storage cells, the other 
pole of which is earthed.* The rod $A$ is held in position by the ebonite plug $P$. This plug fits into a brass ring $B$ connected to earth, and this is in turn held in position by the ebonite ring $C$ fitting into the mouth of the cylindrical vessel. The metallic ring $B$ acts as a guard ring which prevents electricity reaching the electrometer over the surface of the ebonite insulation if faulty. Electricity can therefore

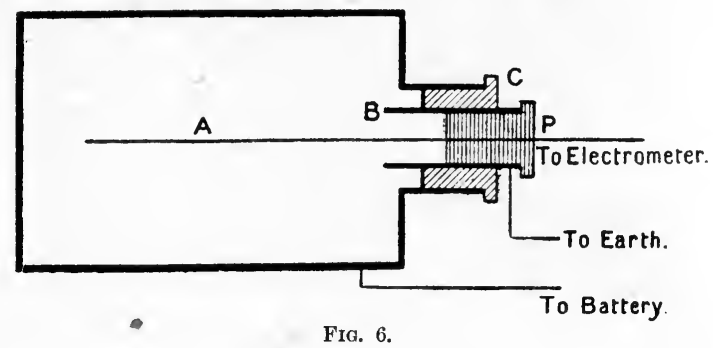

reach the electrometer only through the gas inside the ionisation vessel, and the electric current gives a measure of the ionisation produced in the gas by the radiation passing through it. The rays are either allowed to pass through the walls of the vessel into the gas from a source situated outside, or the source of radiation is introduced into the vessel.

Another form of ionisation vessel which is sometimes used is shown in figure 7. The metal box $M$ has two insulated plates $A$ and $B$ fixed at a distance of a few centimetres from each other; the current flowing between the plates is measured. This form of ionisation chamber is often convenient when the source of radiation has to be introduced into the vessel. This can be done by placing the radio-active material in a shallow dish on the plate $B$. When used in this way, one side of the box $M$ should be made to open to allow the active material to be quickly introduced or removed. In this

* In order to insure a steady voltage, a separate battery of small accumulators is used. Cadmium cells present the advantage of having a small temperature eoefficient, but when using this type of eell, particular care must be taken not to short-circuit the battery. (Kriiger, Phys. Zeitschr. 7. 182, 1906.) 
type of ionisation vessel, the case of the vessel acts as the guard ring.

With penetrating radiations it is impossible to make the ionisation vessel large enough to absorb all the radiation passing into the gas; thus only a fraction of the energy in

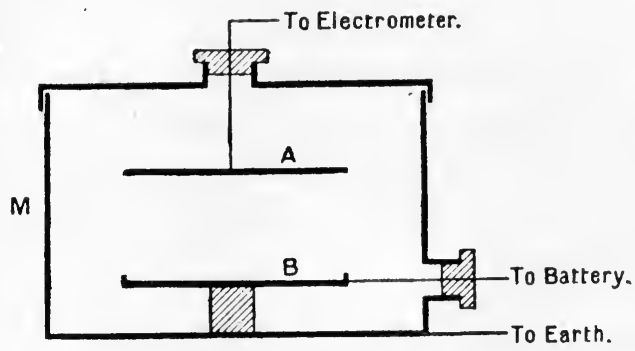

FIG. 7.

the radiation is used. Greater sensitiveness is obtained by using large ionisation vessels; but the advantage thus gained is counteracted by the increase of the natural leak $(\$ 8)$, and the difficulty of producing saturation $(\$ 25)$.

Another way of increasing sensitiveness consists in raising the pressure of the gas to be ionised, but the difficulty of constructing apparatus for the purpose limits the application of the method. If the method is adopted it must be borne in mind that the saturation voltage will be increased $(\$ 25)$.

\section{\$6. The Earthing Key.}

When working with an electrometer, it is necessary to employ specially constructed keys for earthing and insulating the quadrants. A useful form of key is constructed as shown in figure 8 . A brass $\operatorname{rod} R$ with a platinum tip is connected to earth, passes through an earthed metal tube $S$ and makes contact with a small platinum plate mounted on an ebonite block $E$. The platinum plate is connected to the quadrants,

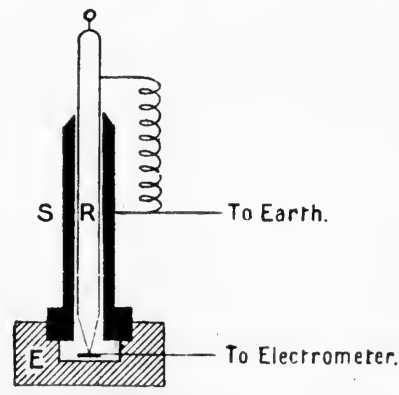

FIG. 8. which are therefore earthed when the rod $R$ touches the 
platinum plate. On raising the rod $R$ the quadrants are insulated, and a current begins to flow from the ionisation vessel into the electrometer. The key and its connections are enclosed in a metallic case to protect them from electrostatic disturbances. The key is raised and lowered by means of a string passing out of the metallic case over a pulley. It is sometimes convenient to substitute for the platinum plate a mercury cup into which dips an amalgamated copper rod.

\section{$\$ 7$. Measurements of Ionisation Currents.}

For making measurements of ionisation currents, the electrometer and all its connections must be enclosed in metallic conductors connected to earth in order to protect them from electrostatic disturbances. The wires leading to the electrometer must not be covered with cotton or other insulating material; they are held in position by sealing wax or ebonite supports. It is best to make the earth connections to the

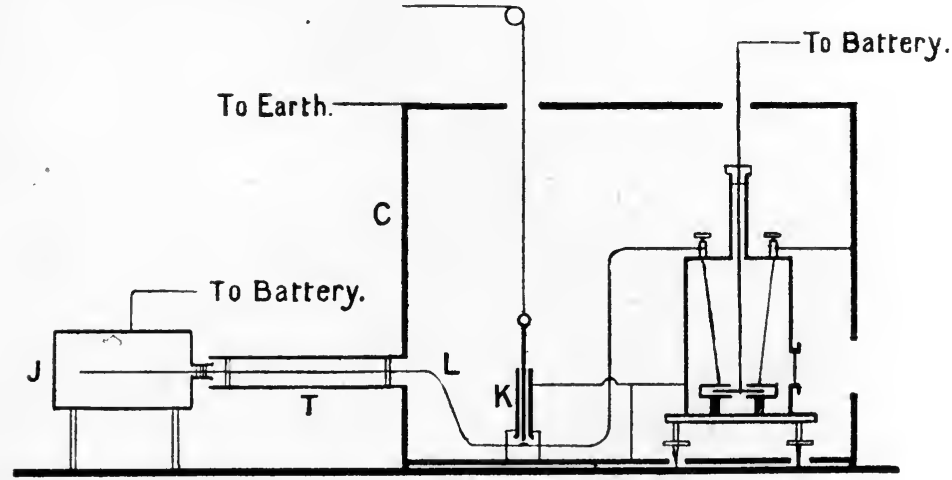

FIG. 9.

water pipes, and care must be taken to insure good contacts by soldering. Lack of attention to this point is often the cause of much trouble and waste of time.

The ionisation vessel $J$ is connected to one terminal of a battery giving at least 100 volts, the other terminal being earthed (fig. 9). A bare wire $L$ passes through an earthed tube $T$ into the electrometer case $C$, and is connected to one pair of quadrants through the earthing key $K$. The rate at which the quadrants charge up is given by the motion of the 
needle, which thus affords a measure of the current passing through the ionising vessel. The simplest way of carrying out measurements when the currents are small is to observe with a stop watch the time required for the electrometer needle to be deflected through a definite angle. After raising the key a short time must be allowed to elapse before starting observations, since the motion of the needle is at first unsteady; as soon as the motion has become uniform, measurements may be begun. This method does not give reliable results unless the motion of the needle is slow and does not exceed about 20 millimetres per minute on a scale placed about one metre from the electrometer. When the motion of the needle is more rapid, it is well to reduce the sensitiveness by inserting a condenser ( $\$ 11)$.

Another way of measuring relatively large currents is to introduce a second key, by means of which the quadrants can be detached from the ionisation vessel at a known time after raising the first key. When the needle has come to rest, the deflection produced in that time gives a measure of the current.

In order to determine the current in absolute units, the capacity of the electrometer and its connections must be determined. This can be done by measuring the current through a gas with and without a capacity of known magnitude in parallel with the electrometer $(\$ 13)$.

Let $C$ be the capacity of the whole system in electrostatic units, $d$ the number of divisions passed over by the spot of light per second and $D$ the deflection obtained for a potential difference of one volt between the quadrants ; then the current is given by the product of the capacity and change of potential per second, and is therefore expressed, in electrostatic units, by the equation

$$
i=\frac{C d}{300 D},
$$

the factor 300 being introduced to convert the value $D$ from volts to electrostatic units.

When the capacity of the system connected with the electrometer is not large in comparison with that of the electrometer 
itself, the method is subject to certain objections; for when the needle becomes charged it changes its position, so that the capacity of the electrometer is apparently increased ( $\$ 2$, equation 7). The above method of using the electrometer is therefore, as a rule, abandoned when currents have to be measured in absolute units.

In order to avoid the difficulty of measuring the capacity of the electrometer system for absolute determinations of current, the zero method described in $\$ 10$ can be employed.

\section{$\$ 8$. Natural Leak of Electrometer and Insulation Tests.}

When an electrometer is connected with an ionisation vessel, as described in $\S 7$, even in the absence of any radio-active matter, a current will flow through the air from the battery into the quadrants on raising the earthing key. This current is known as the natural leak, and is due to the fact that air under normal conditions conducts electricity slightly owing to the presence of traces of radio-active matter in the atmosphere and to penetrating radiation from the earth. In addition to this effect there may be a small leak of electricity over the surface of the insulators in the absence of guard rings. Allowance must be made for the natural leak when measuring the ionisation produced by a source of radiation. This is done by determining the natural leak before and after a measurement and subtracting its value from that of the observed ionisation current. When making a long series of experiments it is well to determine from time to time the natural leak which may not remain perfectly constant. The natural leak should not amount to more than about a hundredth of a volt per minute, when using the electrometer without a condenser.

As the quadrants charge up they tend to lose their charge by leakage through the air and over the insulation. Hence, when measuring small ionisation currents, it will usually be observed that the rate of movement of the needle diminishes somewhat as the deflection becomes greater. 'To test the insulation of the electrometer the quadrants and its connections should be charged to about a volt and insulated. In case the insulation is imperfect the quadrants will gradually lose their 
charge. If the loss does not exceed about a hundredth of a volt per minute the insulation is satisfactory, but if the rate of leak greatly exceeds that amount the insulation should be tested in sections and the faulty piece cleaned or replaced.

When the quadrants are insulated they may gradually charge up even if no voltage is applied to the ionisation vessel. This may be due to two causes. In the first place, if different metals are used in the ionisation vessel, Volta potential differences may appear, but they are without effect when making measurements, since the potential difference applied to the vessel is always great compared with the Volta potential. In the second place, the current may be due to charges previously soaked up by the insulators, and these may gradually be released. This can be rectified by passing a flame over the insulator or, if that is impracticable, by leaving some radium in the neighbourhood for some time to dissipate the charge.

When dealing with penetrating radiations considerable trouble may be experienced on account of the relatively great ionisation produced inside the electrometer and in the spaces between the connecting wires and their earthed cases. The ionisation inside the electrometer can be diminished by placing the source of radiation far away; but this necessitates making the connecting wires long, whereby the ionisation in the spaces round them is increased. To overcome this difficulty, the tubes carrying the connecting wires should be made airtight and then be exhausted. When necessary, the electrometer is placed under a bell-jar from which the air can be removed. With these precautions ionisation currents can occur only in the ionisation vessel. The air in the bell-jar can be reduced to a pressure of a few millimetres of mercury, for the damping of the needle will be unaffected by the change of pressure, since the viscosity of air is independent of the pressure within wide limits.

\section{$\$ 9$. Constant Deflection Method of Measuring Ionisation Currents.}

The method which was devised by Bronson * consists in balancing the current to be measured against a current * Bronson, Phil. Mag. 11.'143, 1906. 
flowing through a known high resistance. The connections are shown in figure 10. $A$ is the ionisation vessel through which the current has to be measured and $B$ is a constant high resistance. The potential of the quadrants of the electrometer will rise until the current flowing through $B$ is equal to that flowing through $A$; the deflection of the needle will then remain constant. If $B$ is a conductor obeying Ohm's law, the current flowing through the ionisation vessel $A$ is proportional to the final deflection of the needle, provided that the scale of the electrometer is uniform.

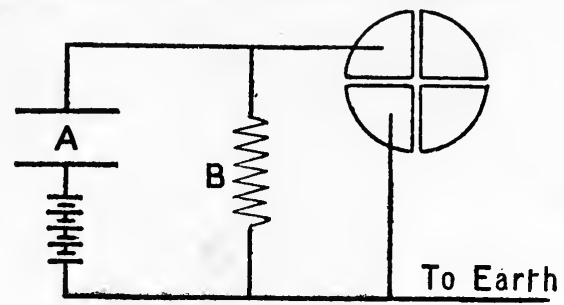

FIG. 10.

This method of determining the current involves the use of a constant high resistance which must be of the order $10^{10}$ ohms. Liquid resistances consisting of a mixture of xylol and alcohol can be used for the purpose.* A mixture containing one part of alcohol dried by lime to ten parts of xylol has a specific resistance of the order of $10^{10}$ ohms per centimetre cube. Gas resistances have also been employed; these can be made by placing some radio-active material on one plate of an ionisation vessel, as shown in figure 7 . The activity of the material selected must remain constant during an experiment, and a polonium film or a layer of uranium oxide usually serves the purpose. So long as the voltage is small the current between the plates will obey Ohm's law, and the deflection will be proportional to the current.

In the application of the method differences of potential arise between the insulated quadrants and earth due to the Volta effect caused by the resistance $B(\S 8)$. The magnitude of the effect can be found by disconnecting the ionisation vessel. On insulating the quadrants, the system will charge * Campbell, Phil. Mag. 23. 668, 1912. 
up until the Volta potential is reached, and the deflection of the needle thus caused must be subtracted from the subsequent observations on the ionisation current produced in $A$. Another method of eliminating the Volta effect consists in counteracting it by applying to the resistance $B$ a potential just sufficient to keep the needle at zero when no current flows through $A$.

\section{$\$ 10$. Zero Method of Measuring Ionisation Currents.}

An accurate method of measuring currents through gases has been devised by Townsend.* The current through the gas between the electrodes $A A^{\prime}$ tends to charge the electrometer; but the charge acquired is continuously balanced by inducing on the insulated system a charge of opposite sign by means of a condenser $K K^{\prime}$ (fig. 11). The plate $K$ is con-

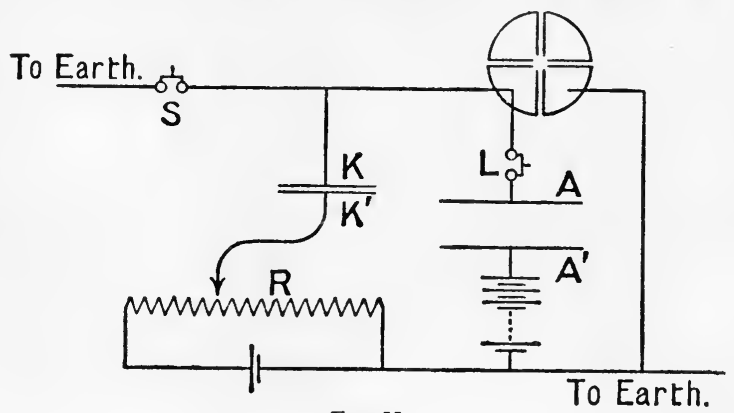

FIG. 11.

nected to the electrometer, and the other plate $K^{\prime}$ is connected to a potentiometer $R$, as indicated in the figure. If the electrometer needle is thus kept undeflected, the ionisation current through $A A^{\prime}$ must be equal to the charge induced on plate $K$ : in unit time.

If $V$ is the potential acquired by the plate $K$ of the condenser in time $t$, the current $i$ flowing through the ionisation vessel is given by

$$
i=\frac{V C}{t}
$$

where $C$ is the capacity of the condenser.

To make measurements, the electrometer is insulated by raising the key $S$. The electrometer needle now begins to

* Townsend, Phil. Mag. 6. 598, 1903. 
move, and the spot of light is brought back to zero and kept there, as nearly as possible, by continuously varying the position of the contact on the resistance $R$. After sufficient time has elapsed the electrometer is disconnected from the ionisation vessel by raising the key $L$. The needle is then brought back exactly to zero by adjusting the point of contact on the resistance $R$. From the position of contact the potential of the condenser is deduced. Hence, if the capacity of the condenser is known, the current can be found from equation 10. Measurements of currents may thus be made without the necessity of knowing the capacity of the electrometer system. The method has been slightly modified by Moulin* and Lattès. $\dagger$

\section{$\$ 11$. Condensers.}

When large ionisation currents have to be measured it is frequently necessary to reduce the sensitiveness of the electrometer. This can be done by connecting to the insulated quadrants of the electrometer one terminal of a condenser the other terminal of which is earthed. The current from the ionisation vessel has then to flow into the condenser as well as into the electrometer, with the result that the quadrants charge up less rapidly. The capacity of a

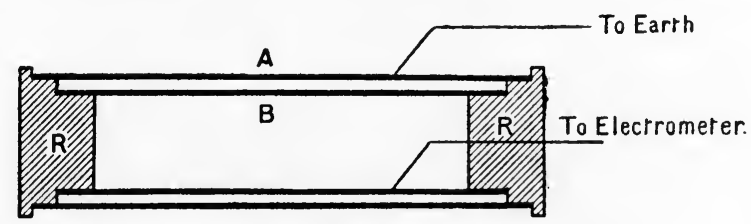

FIa. 12.

Dolezalek electrometer and its connections is usually about $50 \mathrm{cms}$. When a large reduction in sensitiveness is required a sub-divided mica condenser of the ordinary type can be used with advantage. When small capacities are required it is preferable to work with air condensers with small insulating supports of sulphur or amber on account of the trouble due to residual charges taken up by solid dielectrics (§8). A convenient adjustable air condenser has been

* Moulin, Le Radium, 4. 190, 1907. ' + †attès, Le Radium, 6. 73, 1909. 
constructed by Gerdien.* It has a range from about 50 to 500 cms.

An air condenser of fixed magnitude can easily be made as shown in figure 12. The condenser consists essentially of two coaxial brass tubes $A$ and $B$ separated from each other by two ebonite stoppers $R, R$. If the tubes are made long the capacity per unit length can be calculated from the relation

$$
C=\frac{1}{2 \log _{e} \frac{a}{b}},
$$

where $a$ is the internal radius of tube $A$, and $b$ the external radius of tube $B$.

\section{$\$ 12$. Measurement of Capacities of Condensers by Multiple Discharge Method.}

The ordinary ballistic method is not sensitive enough for measuring small capacities such as are used in radio-active measurements; but the determination can be made by alternately and in rapid succession charging the conclenser and then discharging it through a ballistic galvanometer. $\dagger$ The experimental details have been perfected by Fleming and Clinton. +

If the capacity of the condenser expressed in microfarads is $C$, and if it is charged each time to a potential $V$, the galvanometer is traversed by a series of discharges each equal to $C V$ microcoulombs. If there are $n$ discharges per second, the electricity flowing through the galvanometer is equivalent to a steady current of $C V n$ microamperes or $C V n \times 10^{-6}$ amperes. If $n$ is known, the capacity of the condenser in microfarads can then be determined by finding the current which gives the same steady deflection as the intermittent discharge. To convert to electrostatic units (cms.), the quantity obtained must be multiplied by $9 \times 10^{5}$.

The difficulty of the method consists in constructing a suitable interrupter without introducing capacity into the system. An electrically driven tuning fork of known pitch can be

* Gerdien, Phys. Zeitsch. 5. 294, 1904.

†J. J. Thomson, Phil. Trans. 718, 1883.

R.-A.

¥Fleming and Clinton, Phil. Mag. 5. 493, 1903. 
used to produce an intermittent current which is sent through an electromagnet, as shown in figure 13. The steel plate $A$ is alternately attracted and released by the magnet and causes contact with the two stops $\alpha$ and $b$. Thus the arrangement

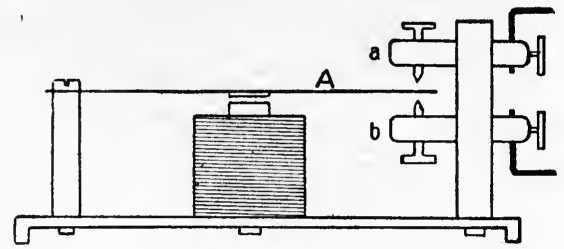

FiG. 13.

shown can be used to charge and discharge a condenser through a galvanometer with a period which is controlled by that of the tuning fork.

\section{$\$ 13$. Measurement of Capacities by Ionisation Currents.}

A rough determination of the magnitude of a capacity can conveniently be made by means of a quadrant electrometer. The method can be used to measure the capacity of the electrometer and its connections, and then to compare other capacities with each other. The measurements are carried out in the following way. An ionisation vessel is connected to the electrometer in the usual manner, and the current produced by a constant source of some radiation, such as uranium oxide, is measured by the electrometer. If $Q$ is the quantity of electricity flowing through the ionised gas in unit time, and if $C$ is the capacity of the electrometer and its connections, the potential to which the system charges up in unit time is equal to $\frac{Q}{C}$. This quantity must be proportional to the deflection $\theta_{1}$ suffered by the electrometer needle. Hence

$$
\frac{Q}{C}=k \theta_{1}
$$

The condenser $B$ of known capacity $C^{\prime}$ is then connected to the electrometer, and the rate at which the quadrants charge up is again measured. The current flowing into the electrometer system being the same as before, the potential 
acquired in unit time will now be $\frac{Q}{C+C^{\prime}}$. If the deflection is $\theta_{2}$, we have the relation,

$$
\frac{Q}{C+C^{\prime}}=k \theta_{2} \text {. }
$$

Combining equations (12) and (13) we obtain the relation,

$$
\frac{C+C^{\prime}}{C}=\frac{\theta_{1}}{\theta_{2}}, \ldots
$$

whence $C$ can be found in terms of $C^{\prime}$.

When the capacity of the electrometer has been determined, any two capacities can at once be compared. The method, though simple, is not very accurate; for unless the capacities are nearly equal, the rates of motion of the electrometer, with and without the condenser, differ so much that the comparison cannot be made accurately. In addition, errors may be introduced on account of the variation of the capacity of the electrometer as the needle is deflected (\$2), unless the currents are measured by noting the time taken for the same deflection of the needle to be produced in each case.

\section{$\$ 14$. Induction Method of Comparing Capacities.}

An accurate comparison of the capacities of two condensers can be made by connecting them both to a quadrant electrometer, and inducing equal but opposite quantities of electricity

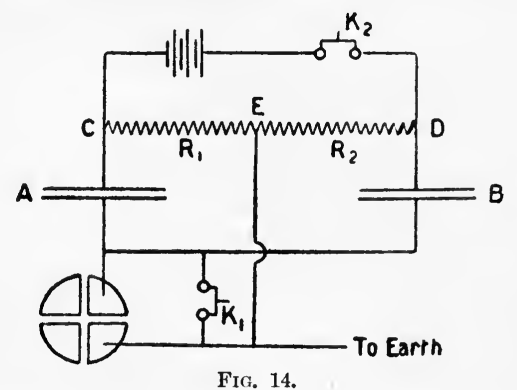

on the condensers. The electrometer will then remain undeflected. By measuring the potentials applied to each condenser to induce the charges, the capacities can be compared.

The experimental arrangement is shown in figure 14. One 
terminal of each of the condensers $A$ and $B$ to be compared, is connected to the same pair of quadrants of an electrometer which can be connected to earth, or disconnected by the key $K_{1}$. The other terminals of the condensers are connected to the two ends $C$ and $D$ of the adjustable resistances $R_{1}$ and $R_{2}$ through which a current can be maintained from a battery giving a few volts. The point $E$ is connected to earth. On inserting the key $K_{2}$ the condensers will be charged respectively to potentials $V_{1}$ and $-V_{2}$. The relation between the two potentials is given by the equation,

$$
\frac{V_{1}}{V_{2}}=-\frac{R_{1}}{R_{2}}
$$

Now if $C_{1}$ and $C_{2}$ are the capacities of the two condensers, the quantities of electricity flowing into each, while charging up, will be $V_{1} C_{1}$ and $-V_{2} C_{2}$, and if these two quantities induce charges which neutralise one another, the electrometer will show no deflection. Hence in these circumstances,

$$
\frac{C_{1}}{C_{2}}=-\frac{V_{2}}{V_{1}}=\frac{R_{2}}{R_{1}}
$$

If therefore the resistances $R_{1}$ and $R_{2}$ are adjusted until no deflection is shown by the electrometer on inserting the key $K_{2}$, the ratio of the two capacities can be found from equation (16). The sensitiveness of the method can be doubled by using a key at $K_{2}$, which reverses the current instead of making it.

The advantage of the method consists in the fact that condensers of widely different magnitude can be compared so that the capacities of small condensers can be found in terms of large ones which can be standardised by any of the ordinary well known methods. It should, however, be remembered that the value of the capacity obtained by different methods for the same condenser may be somewhat different on account of the soaking up of charges by the insulation. The effect is least marked in condensers with air or good mica as dielectric. 


\section{CHAP'TER II.}

\section{ELECTROSCOPES.}

\section{\$15. Alpha-ray Electroscopes.}

A very convenient form of $\alpha$-ray electroscope of moderate sensitiveness, devised by Rutherford, is shown in figure 15 . The instrument consists essentially of a parallel plate condenser $A B$ contained in a metal case, one side of which can be opened to introduce the radio-active material; the lower plate is connected to the case of the instrument, which is earthed, and the upper plate is connected to the rod $C$, which passes through the insulating plug of sulphur $S$ into a second chamber in which a gold or Dutch-metal leaf $L$ is mounted. The leaf is attached to the $\operatorname{rod} C$, which is flattened on one side. The leaf can be charged by removing the cap $K$. The movement of the leaf is observed by means of a microscope through a window $W$ cut in the case as indicated in the figure. Another window is provided on the opposite side of

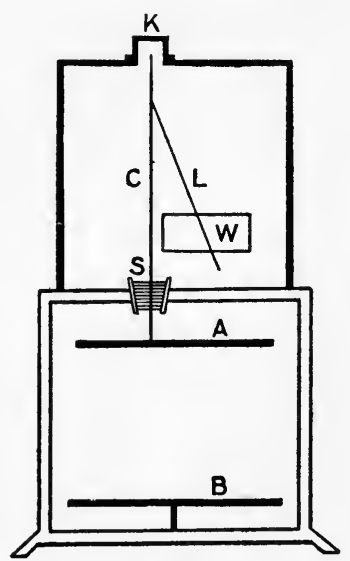

FIG. 15. the case to admit light.

When very feeble sources of $\alpha$ radiation have to be measured, the instrument described above is not suitable, since its capacity is relatively great (about $12 \mathrm{cms}$.). For such measurements a more sensitive instrument can be constructed as shown in figure 16. The case of the instrument may be cubical or cylindrical and its height should be about $8 \mathrm{cms}$. to take in the whole range of the $a$ particles ( $\$ 31$ ). 
The electroscope case and the $\operatorname{rod} A$ supporting the goldleaf system are permanently connected to earth. The leaf is insulated by a small bead of sulphur $S$ and can be charged by contact with the $\operatorname{rod} B$, which can be turned in the ebonite plug $C$. The part $D$ of the charging rod making contact should be made either of easily flexible wire or else a stop should be provided to prevent the support

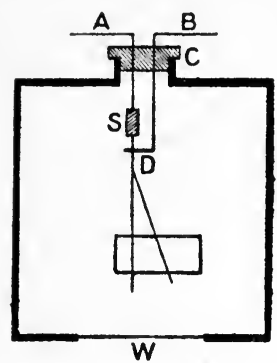

Fig. 16. of the leaf being displaced when being charged; otherwise the capacity and calibration of the instrument may be changed. By rotating the $\operatorname{rod} B$, it is disconnected from the leaf and then earthed. The active matter is brought immediately below the window $W$, which is made of thin aluminium leaf so that the $\alpha$ rays can penetrate into the electroscope without appreciable absorption. The motion of the leaf is observed as in the other type of electroscope with a microscope through suitably placed windows.

In many cases it is preferable to place the active matter inside the electroscope, for which purpose the base of the electroscope must be made removable.

\section{$\$ 16$. Beta and Gamma-ray Electrosicopes.}

The electroscopes used for $\beta$ and $\gamma$ rays are similar to the second type of instrument described above for work with $\alpha$ rays (fig. 16). In the case of $\beta$ rays, however, the very thin aluminium window is replaced by one just thick enough to stop the $\alpha$ particles, for which purpose a thickness of about $.05 \mathrm{~mm}$. is required. The radio-active preparation is placed directly in front of the aluminium window or at some distance away, according to the strength of the material examined.

In the case of measurements with $\gamma$ rays, the electroscope is covered with lead two millimetres thick to protect it on all sides from $\beta$ radiation. It is not sufficient merely to insert a lead screen between electroscope and source, for serious errors may be introduced by excited $\beta$ rays produced by the $\gamma$ rays impinging on objects near the electroscope. For the same 
reason the windows of the electroscope should be of thick glass, which may in addition be screened by lead frames projecting over them. Unless for special reasons, the edges of the case of a $\beta$ or $\gamma$ ray electroscope should be not longer than about 10 centimetres.

\section{$\$ 17$. Methods of making Measurements with Electroscopes.}

For sensitive electroscopes the leaf should be one or two millimetres wide and three or four centimetres long. To make observations with ease and accuracy, a tele-microscope with a large field of view and giving a magnification of about 10 is used. The eyepiece is fitted with a scale of about 80 divisions, which should be about one-tenth of a millimetre apart. The graduations should be not too fine.

The motion of the leaf is observed by focussing on one of its edges with the observing microscope; or an exceedingly thin fibre of glass or quartz may be attached to the lower extremity of the leaf with a minute particle of sealing wax or paste. This gives a very sharp object upon which to focus; : but if this method is adopted, care must be taken to make the fibre and wax used to attach it as light as possible, so as not to detract from the sensitiveness of the instrument.

The leaf is illuminated by means of a lamp placed at some distance from the electroscope. If the lamp is too close, convection currents may be set up in the electroscope, so that the leaf becomes unsteady. It is sometimes better to reflect the light into the electroscope from a distant source of illumination.

The charging of the leaf can be effected by means of an electrified rod of sealing wax or ebonite; but the use of a battery, giving about 300 volts is preferable, since in this way the leaf is always brought to the same division on the scale of the microscope.

When taking measurements the most accurate procedure is, whenever possible, to measure with a stop-watch the time which the leaf takes to pass over a definite number of divisions. This eliminates errors due to lack of uniformity of the scale. This course is, however, impracticable when measuring a weak source of radiation, the activity of which 
varies rapidly. Since in this case measurements at different parts of the scale have to be compared, the scale must be calibrated (\$18). The rate of motion of the leaf over the scale should not exceed 40 divisions per minute. For the reason given in $\$ 21$, the electroscope should not be used immediately after charging the leaf for the first time. On account of the simplicity of their construction and the ease with which measurements can be made with electroscopes, these instruments are, whenever possible, used instead of the quadrant electrometer. The sensitiveness of an electroscope is very great, and with care measurements can be made with an accuracy of 0.5 per cent. Moreover, a variation of activity of one to fifty can easily be determined.

\section{$\$ 18$. Calibration of the Scale of an Electroscope.}

Before starting measurements with an electroscope, it is necessary to see whether the leaf moves uniformly by ascertaining the sensitiveness of the instrument at different parts of the scale. To do this some uranium-oxide or other constant source of radiation is placed in or near the electroscope, and the rate of motion at different parts of the scale is observed by noting the time of passage of the leaf over successive scale divisions. To get accurate results, the intensity of the radiation should be such that it will give a rate of discharge of 5 to 10 divisions per minute. If much slower than this, the measurements take an inconveniently long time, whereas if the rate is much greater, the observations become inaccurate.

Having calibrated the scale in this way, it is a simple matter to express all the observations subsequently made with the electroscope in terms of some particular scale divisions taken as standard. The lack of uniformity of the scale should not amount to more than five per cent. from end to end.

\section{$\S 19$. Natural Leak of Electroscopes.}

As with the quadrant electrometer, allowance must be made for the natural leak when making measurements with an electroscope (\$8). The natural leak must be determined 
before and after making measurements. This is necessary because it is apt to vary slightly owing to changes in the insulation, and also on account of possible contamination of the instrument with radio-active material. The electroscope, after being charged, is left for about a quarter of an hour, and the change of the position of the leaf in this time noted. It is advisable to keep the electroscope charged for some minutes before starting measurements; for disturbances are apt to occur when the leaf is first charged (§21). If the insulation of the leaf is good and the apparatus is otherwise working satisfactorily, the leaf will not move more than about a tenth of a division per minute even with an electroscope of small capacity. If the natural leak is much higher than this, the sulphur insulation should be cleaned by scraping with a pen knife; and if this is not effective in reducing the leak to its normal value, the inside of the electroscope should be cleaned to remove any adhering radio-active matter. It may happen that the natural leak is increased by the presence of radium emanation in the room, which must then be thoroughly ventilated. To exclude emanation from the electroscope it should, if possible, be made air-tight.

\section{\$20. Electroscope Leaves.}

In sensitive electroscopes gold leaves are used; for these give the greatest sensitiveness and are more satisfactory than leaves of other metals. The difficulty of cutting gold leaves has, however, led to the use of aluminium and Dutch-metal, which have the advantage of being more easily cut and mounted. On account of the lightness of aluminium, leaves of this metal are frequently employed, but for accurate measurements their use is to be discouraged because of their rigidity. 'The leaf, instead of gliding uniformly across the scale of the microscope, tends to move in jerks. Dutch-metal is much less objectionable in this respect.

Dutch-metal and aluminium leaves can be cut between two sheets of paper with a good pair of scissors and mounted without much trouble, whereas the cutting and mounting of gold leaves require some skill in manipulation. Some 
hints as to the best way of handling the leaves may therefore be of use. To cut the gold leaf, it should be placed between two grease-free pieces of paper taken from the book in which the leaves are supplied. The leaf is then laid flat on a pad of blotting-paper and cut with a single stroke of a razor. It is usually best to cut leaves one or two millimetres wide and three or four centimetres long. The leaf must be carefully removed from the grease-free paper and attached with a small drop of paste or shellac to the brass support which is to hold it in position in the electroscope. Care must be taken to place the leaf straight on its support, as its position cannot be changed when once it has been attached. The brass strip holding the leaf must be very smooth and clean, and well polished, as the leaf will otherwise stick to the support.

Another method of cutting gold leaves has been devised by Beatty.* Though somewhat tedious, the method when properly employed gives very good leaves. The details are as follows:-A clean glass plate is coated with a thin layer of paraffin wax by melting it over the surface. The gold leaf is then placed on the wax so as to lie flat, and is made to adhere to the wax by slightly warming the under surface of the glass. Some particles of paraffin are then dusted on the surface of the leaf and melted over it so as to embed it in wax. When the wax has solidified the leaf can be cut up into strips and these removed from the plate by warming and gently lifting with a pin. The leaves are then placed in xylol, and when the paraffin is completely dissolved they are transferred to a bath of alcohol. The transference is made on a sheet of paper which prevents the leaves from being crumpled, in drawing them through the surface of the liquid.

\section{$\$ 21$. Insulators.}

For work with electrostatic instruments of low capacity, the only available insulators are ebonite, sealing-wax, fused silica, sulphur and amber, of which the last two are by far the most suitable for gold-leaf electroscopes. Fused silica,

* R. T. Beatty, Phil. Mag. 14. 604, 1907. 
which is an excellent insulator when pure, must be selected with great care as different specimens vary considerably in insulating powers. Silica is, however, of great use at moderately high temperatures, as it is the only insulator which can be appreciably heated.

Amber can be obtained either in its natural state or in the form of "Amberite" or "Amberoid," which are the names under which certain artificially prepared kinds of compressed amber dust are put on the market. In any of these forms amber is excellently suited for work with electrostatic instruments.

Sulphur is most commonly used for the insulation of gold-leaf electroscopes. It is second to nothing in insulating power, and can be prepared in any desired shape by melting and casting in glass or paper moulds. Care must be taken not to overheat the sulphur when melting, otherwise it will be found on solidification to have lost its insulating power; the sulphur should be allowed just to melt by slowly heating, when the liquid can be sucked into a glass tube and allowed to set; after solidification the sulphur gradually contracts and can be pushed out of the glass tube after some hours. The insulating power improves for some time after casting. When mounted in the electroscope, the sulphur bead must be situated so as to be protected from direct daylight; for under the influence of light, it is apt to lose its power of insulation.* Whatever insulation is used trouble will be experienced in delicate work with electroscopes due to charges soaking into the insulation. For this reason irregular readings are often obtained immediately after charging up an electroscope. Some insulators are much worse than others in this respect, and paraffin, which is an excellent insulator, is quite useless for work with electroscopes and electrometers on this account. With amber and sulphur the effect is relatively small, but still large enough to give trouble when making delicate measurements. The effect is diminished by reducing the size of the insulator, so that it is very important to make the insulating supports small.

* Bates, Le Radium, 8. 312, 1911. 


\section{\$22. Emanation Electroscopes.}

It is frequently necessary to estimate small quantities of radium emanation, and for this purpose a special type of electroscope is necessary. The emanation is introduced into the electroscope itself, or into an ionisation chamber connected to it. There are two types of instrument in general use, which are shown in figures 17 and 18. In figure 17 a special ionisation chamber $A$ can be evacuated through the tap $C$, and the gas to be tested admitted into the chamber through the $\operatorname{tap} B$. Atmospheric pressure is established in the vessel $A$ before taking readings. The ionisation is measured by the motion of the gold leaf $L$ which is attached to the rod $R$. The insulation is effected by the sulphur plug $S$, which serves to support the gold-leaf system.

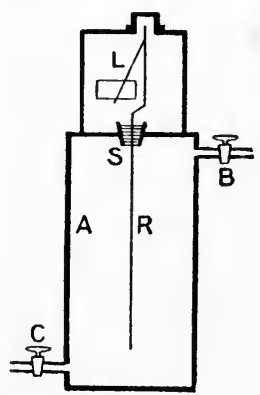

FIG. 17.

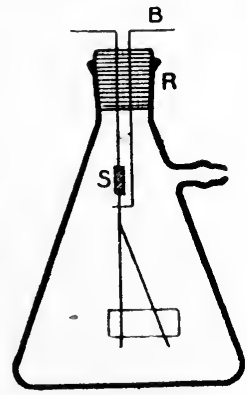

FIG. 18.

In the second type of instrument (fig. 18), the emanation is admitted directly into the chamber containing the gold leaf, which may be an ordinary filter-flask silvered inside. Two regions are scraped free of silver to serve as windows, and the gold leaf which is insulated by the sulphur $S$ is held by the rubber stopper $R$. A strip of tin foil placed between the stopper and the neck of the flask, serves to earth the silver surface. A charger $B$ also passes through the stopper $R$, and by rotation can be brought into contact with the support of the gold leaf. If this type of instrument is used, great care must be taken to evacuate and re-admit the air slowly; for if this precaution is not taken, the gold leaf may be blown off in the process. To guard against this happening, it is 
best to evacuate and re-admit the air through a fine capillary tube.

The details of estimating quantities of radium emanation are given in $§ 62$.

\section{\$23. The Tilted Electroscope.}

A very sensitive electroscope which has advantages over the electroscopes already described for making certain measurements has been constructed by C. T. R. Wilson* and refined in some of its details by G. W. C. Kaye. $\dagger$ This type of instrument has to be used in conjunction with an ionisation chamber. The advantages of the instrument are that, compared with a quadrant electrometer, it has a very small capacity amounting to only a few centimetres, and that it can be made very sensitive. The instrument is shown in figure 19.

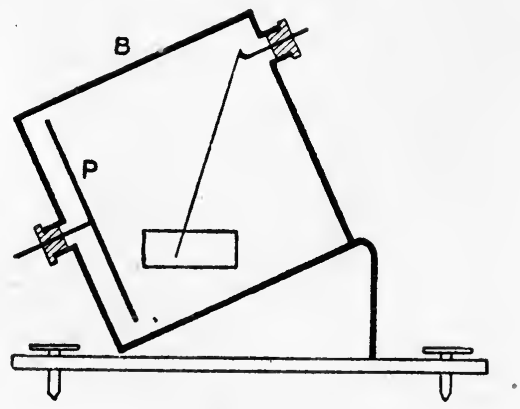

Fid. 19.

The rectangular metal case $B$, which is earthed, contains within it a plate $P$, which is connected to one terminal of a battery, the other terminal of which is earthed. The instrument can be tilted to any desired angle. The position of the leaf is observed by a microscope through a window in the case. The leaf is connected through a key to the ionisation vessel in which the current is to be measured and is maintained at earth potential until the measurement begins; it is then disconnected and allowed to charge up. Its position will change as its potential rises.

* C. T. R. Wilson, Proc. Camb. Phil. Soc. 12. 135, 1903.

† G. W. C. Kaye, Proc. Phys. Soc. Lond. 23. 209, 1911. 
For any particular tilt of the instrument there is a difference of potential between the gold leaf and plate, for which the sensitiveness is a maximum. This difference of potential is greater for small than for large angles of tilt; but when too little tilted, the leaf becomes unstable over a part of its range of motion. The angle at which this takes place is known as the critical inclination. As the instrument is less and less tilted, so that the plate becomes more nearly vertical, the range of instability becomes greater. In using the instrument the greatest sensitiveness is obtained when the angle of tilt is slightly greater than the critical inclination.

To adjust the instrument it is tilted through about $30^{\circ}$. The potential on the plate $P$ is varied until the maximum sensitiveness for that angle is obtained. For that purpose about 200 volts are usually needed. If the maximum possible sensitiveness is required, the maximum sensitiveness must be found for different angles of tilt and the instrument set up at the most favourable angle. It will be found that the instrument is used with the leaf nearly in a position of instability. It follows, therefore, that although the electroscope is very sensitive, the deflections of the leaf are not proportional to the voltage applied to it. Readings must therefore be taken only over a definite number of divisions or the scale must be carefully calibrated.

On account of the great sensitiveness of the electroscope it is very susceptible to change in the potential of the plate $P$. For this reason cells giving a constant voltage must be used to charge the plate. It is therefore best to use a battery of cadmium cells, which give a very steady potential (see footnote, p. 8).

\section{\$24. Quartz-fibre Electroscope.}

A quartz-fibre electroscope has been designed by Wulf* and its construction is shown in figure 20. It consists essentially of two quartz-fibres 5 or 6 cms. long suspended from an insulated rod so as to hang very close to each other. The lower ends of the fibres are connected to a small piece of tin foil which acts as a light weight. The fibres are made conducting * Wulf, Phys. Zeitschr. 8. 246, 527 and 780, 1907. 
by spluttering platinum on to them from the cathode of a discharge tube.* When the fibres are charged they repel each other, forming a loop whereby the small weight attached to them is slightly raised. The distance between the fibres is measured by a microscope, and is very nearly proportional to the potential to which they have been charged.

The chief advantage of the instrument over the ordinary gold-leaf electroscope consists in the fact that it is almost dead-beat. For further details the reader is referred to

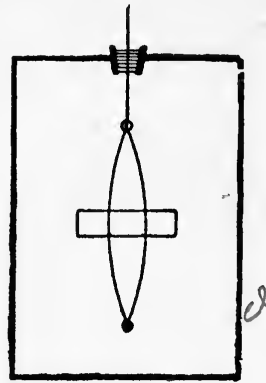

Fig. 20. the original paper in which the instrument is described.

* Bestelmeyer, Zeitschr. für Instrumentenkunde, 25. 339, 1905. 


\section{CHAPTER III.}

THE IONISATION OF GASES.

\section{$\$ 25$. The Ionisation of Gases by Radiations.}

When gases are traversed by certain radiations, as for example cathode or Röntgen rays, they are rendered conductors of electricity. The same result is produced by the radiations from radio-active substances, and on this property is based the method usually adopted to detect radio-active bodies and measure the intensities of the radiations emitted by them. On this account it is important to study the laws governing the conduction of electricity by gases before considering the phenomena of radio-activity in detail.

When an ionised gas is subjected to an electric field, there passes through it a current which, so long as the field is not great, is proportional to the difference of potential between the electrodes immersed in the gas: in other words, the gas behaves like a metallic conductor and obeys Ohm's law. When the potential difference is increased beyond a certain limit, the current rises less rapidly and ultimately tends to a limit, thus becoming independent of the voltage applied. This limiting value is known as the saturation current.

This phenomenon has been explained by supposing that the ionising agent continuously produces from the gas positive and negative ions which, by moving to the electrodes, convey the current. In the absence of an electric field, the ions recombine and neutralise each other, and a steady state is reached when the rate of recombination is equal to their rate of production. Under the influence of an electric field, some of the ions are dragged to the electrodes before recombining, and if a large enough field is applied, the ions are 
withdrawn by the field as soon as they are formed. The current through the gas then ceases to rise with increase of voltage, and saturation is reached. In these circumstances the current gives a measure of the intensity of the ionising agent.

When comparing radiations, saturation currents should be used and tests must be carried out to make sure that the potential difference applied is sufficiently great. It is difficult to foresee what potential will be required in any specified case, since the voltage necessary to produce saturation depends on a number of complicated factors.

In the first place the saturation voltage increases with the intensity of radiation; for, as the concentration of the ions in the gas grows, "the rate of recombination becomes greater. Accordingly a somewhat higher voltage is required to remove the ions before they recombine.

The dimensions of the ionising vessel will also influence the electric intensity required for saturation; for when the electrodes are far apart the ions will have a great distance to travel before reaching the electrodes, and will therefore have to move very rapidly if they are to escape recombining in traversing the gas. It is evident that, as the distance between the electrodes increases, the value of the saturation current as well as the saturation intensity increases; for the total number of ions withdrawn from the gas is obviously larger. The shape of the vessel also influences the saturation voltage. Thus it is more difficult to obtain saturation in a cylindrical ionisation vessel with a thin axial electrode than in one provided with parallel plates; for in the former case the electric force falls off rapidly with the distance from the central electrode. Hence, although the field may suffice to remove all the ions formed near that electrode, this may not be the case at greater distances from it.

As might be expected, the saturation voltage depends on the nature of the gas ionised. Moreover it has been shown that the presence of dust or moisture retards the velocity of the ions, and so increases the difficulty with which they are removed by an electric field.

R.-A. 
A somewhat remarkable difference exists between the saturation voltages required with different radiations, even when all the conditions considered above are fixed. Thus, for the same intensity of radiation as measured by the saturation current in a particular apparatus, the saturation voltage will be greater for $\alpha$ than for $\beta$ rays; for an $\alpha$ particle produces far more ions than a $\beta$ particle per centimetre of its path. The local concentration of the ions produced by $\alpha$ rays is therefore much greater than that produced by $\beta$ rays; for the same total ionisation, a much greater voltage is therefore required to produce saturation with $\alpha$ than with $\beta$ rays. The effect can be studied by means of an ionisation vessel, as shown in figure 6 . Typical curves obtained with $\alpha$ and $\beta$ rays of the same intensities are given in figure 21 .

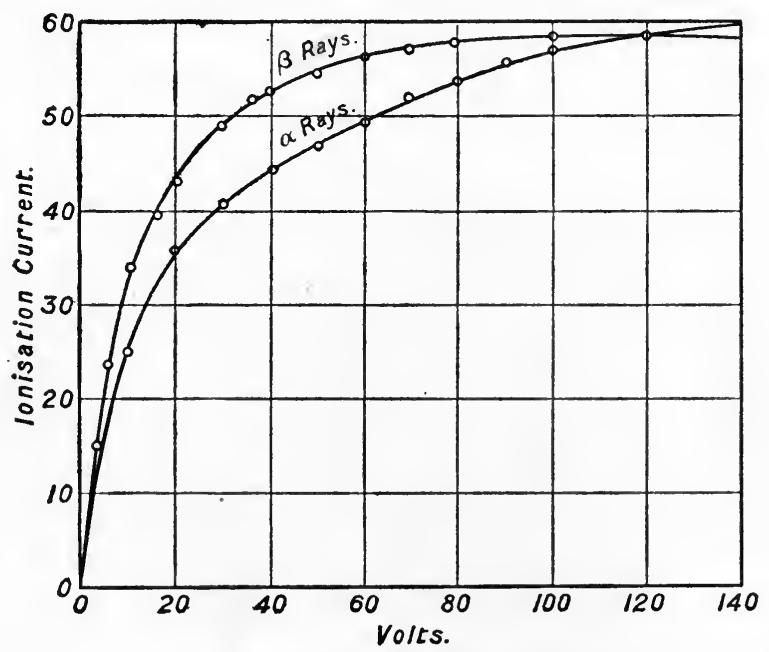

Fra. 21.-Saturation curves for $\alpha$ and $\beta$ rays.

\section{$\$ 26$. Rate of Recombination of Ions. *}

An ionised gas retains its power of conducting electricity for some time after the ionising agent has been removed. This is due to the fact that an appreciable time is required by the ions to recombine. Now, since recombination depends on the encounter of two ions, the rate of recombination will 
be proportional to the square of the number of ions present. Thus, if $n$ is the total number of ions present at any moment,

$$
\frac{d n}{d t}=-\alpha n^{2}
$$

where $\alpha$ is a constant known as the coefficient of recombination. Hence, if $n_{1}$ is the number of ions present at a definite time and $n_{2}$ the number present at any subsequent time $t$,

$$
\frac{1}{n_{2}}-\frac{1}{n_{1}}=\alpha t
$$

The rate of recombination of the ions can be studied and the law expressed by equation (18) tested by a method first used by Rutherford.* It consists essentially in determining the decrease with time of the number of ions when a stream of ionised air is passed through a tube at a constant rate, the saturation current being determined at different positions along the tube. The apparatus is shown in figure 22 .

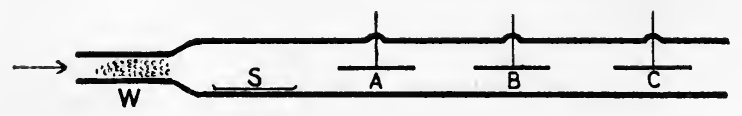

FIG. 22.

By means of a large gas holder or the arrangement described in $\$ 55$, a constant stream of air is first passed over a drying agent and then through the plug of cotton wool $W$ to remove dust. The air then passes over some uranium oxide placed at $S$ and is ionised. The number of ions present will diminish as the gas passes along the tube. By means of the wires $A, B, C$, the saturation current can be measured at different distances from the uranium. The wires are in turn connected to a quadrant electrometer and a sufficient voltage applied to the tube to produce saturation. The wires not connected to the electrometer are maintained at the same potential as the tube. The current at the different positions in the tube is determined in absolute measure. Taking the charge carried by a single ion as $4.65 \times 10^{-10}$ E.s. unit, the numbers of ions $n_{1}$ and $n_{2}$ present in the gas at different intervals of time after leaving the uranium can be calculated.

* Rutherford, Phil. Mag. 47. 109, 1899. 
The time interval $t$ which elapses while the air flows from one wire to the next can be deduced from the rate of flow of the gas. From equation (18), the coefficient of recombination $\alpha$ can then be calculated. For air $\alpha$ has the value $10^{-6}$ approximately.

In addition to the loss of ions by recombination there is a further loss due to diffusion of ions to the sides of the tube. But if the tube is not too narrow, the loss due to diffusion will be small compared with that due to recombination. The fact that air can be completely freed from ions by blowing it through cotton wool is due to diffusion, for the interstices of the cotton wool are small and the chance of the ions losing their charges by diffusion correspondingly great.

\section{$\$ 27$. Ionisation by Collision.*}

Radiations such as the cathode, $\alpha$ and $\beta$ rays consist of charged particles moving with high velocity. It might therefore be expected that the ions in a gas would themselves produce ionisation if caused to move sufficiently quickly. Under the influence of a strong electric field, the ions move rapidly towards the electrodes, and it has been shown by Townsend that when the velocity is great enough the negative ions begin to produce new ions in their paths. Now for a constant difference of potential the speed with which the ions move through the gas is inversely proportional to the pressure if this is not too small; but at pressures which do not exceed a few centimetres of mercury, the speed of the negative ion increases very rapidly with decrease of pressure. This is explained by supposing that at low pressures the electron initially liberated in the process of ionisation moves freely through the gas, whereas at high pressures it attaches itself to a gas molecule. The electron is thus converted into a negative ion, and on account of its much larger mass moves more slowly in an electric field. Hence, at low pressures a smaller field than at high pressures suffices to produce ions by collision. Since the positive

* Rutherford, Radio-activity, 1912, § 15.

† Townsend, The Theory of Ionisation of Gases by Collision, Constable \& Co., London, 1910. 
charge is always associated with a large mass, the positive ions are less efficient in producing ionisation by collision, and are only able to do so when the sparking potential is nearly attained.

The effect can be demonstrated and studied by means of the apparatus shown in figure 23. An insulated wire $W$ is fixed along the axis of the cylindrical vessel $V$, which is kept at a positive potential by a battery of accumulators. The vessel is exhausted through the tube $T$ to a pressure of about one centimetre of mercury. A potential of about 1 volt is

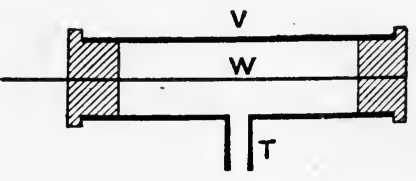

FrG. 23. applied to the vessel, and the current through the gas measured by an electrometer. On increasing the potential the current at first increases, as described in $§ 25$, finally reaching saturation. If, however, the potential is still further increased, the current begins again to rise rapidly; ionisation by collision has then begun to take place.

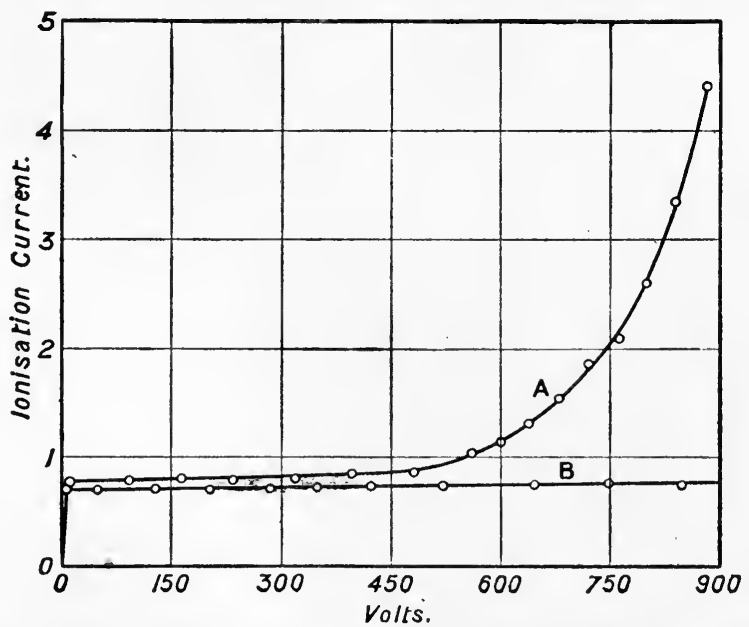

F19. 24.-Variation of ionisation current with intensity of field (ionisation by collision).

Some typical results are shown in figure 24. The curves were obtained with an ionisation vessel of $2 \mathrm{cms}$. diameter, provided with an axial rod of $5 \mathrm{mms}$. diameter. The pressure 
of the air was $3 \mathrm{cms}$. of mercury and ionisation by collision began with about 400 volts. If the pressure of the air is still further reduced, and if the central electrode is much thinner, ionisation by collision sets in at a lower voltage.

Curve $A$ refers to the case in which the tube was charged negatively, and $B$ to that in which the tube was charged positively. It will be seen that the curves are very different in shape. The difference between the two cases is due to the fact that in the first case the negative ions are drawn into the intense field round the wire, and produce ionisation by collision at a comparatively small voltage. In the second case the positive ions travel into the intense field, and it has already been pointed out that the positive ions are inefficient in producing ionisation by collision.

\section{$\$ 28$. Measurement of the Ratio of the Charge to the Mass and of the Velocity of Cathode Rays.*}

The corpuscular radiations occurring in radio-activity are characterised by the mass, the charge and the initial velocity of the particles composing them. The mass of the particles cannot be measured directly, and the determination of the charge carried by each particle presents great experimental difficulties; for, although it is a comparatively simple matter to measure the charge carried by the radiation from a definite source, the estimation of the number of particles constituting the radiation involves the use of special complicated methods. But the ratio of the charge to the mass can be deduced, as is shown below, from measurements of the deflection of the particles in electric and magnetic fields. The same method also affords a means of determining the velocity with which the particles move.

On account of the weakness of the sources of radiations usually available, it is troublesome to carry out measurements with radio-active substances. The method of procedure is, however, exactly analogous to that adopted for cathode rays, with which the determination can be made more easily.

* Rutherford, Radio-activity, 1912, $\S 30$ and 31. See also J. J. Thomson, Conduction of Electricity Through Gases, 2nd edition. 
In the cathode stream the electrons are projected in straight lines, from which course they can be deflected by an electric or a magnetic field, on account of the charge which they carry with them. Suppose that the cathode rays are produced in a tube such as is shown in figure 25, so as to fall

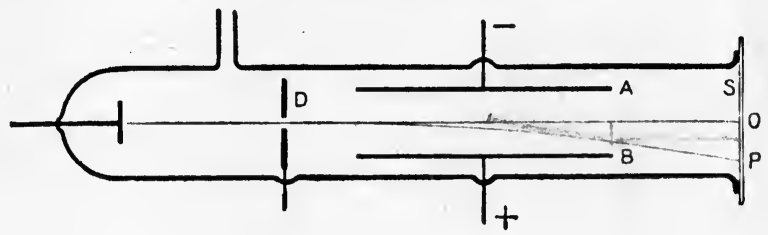

FIG. 25.

upon the phosphorescent screen $S$ mounted on a glass plate attached to the end of the tube. The rays pass through a slit $D$ to confine the beam and produce a narrow band of phosphorescence, the position of which on the screen $S$ is noted. If now an electric field is applied between the two plates $A$ and $B$, the rays will be deflected from their path, and the phosphorescent band of light produced by them will be displaced. A tube $25 \mathrm{cms}$. long and $5 \mathrm{cms}$. wide with plates mounted $2 \mathrm{cms}$. apart is suitable. The width of the slit should be less than a millimetre.

Let $l$ be the length of the plates between which the electric field is maintained, and let $k$, their distance apart, be small compared with $l$. Since the field is uniform between the plates, the problem is exactly similar to that of a body projected horizontally and acted on by the force of gravitation; the electrons will therefore move in a parabola. Let $F$ be the electric force maintained between the plates, $e$ the charge carried by the electron and $m$ its mass. The acceleration of the electrons towards the plate $B$ is $\frac{F e}{m}$, and the displacement $x_{1}$ suffered by the particles on reaching the end of the electric field is given by

$$
x_{1}=\frac{1}{2} \frac{F e}{m} t^{2},
$$

where $t$ is the time which the particles take to pass through 
the electric field. Introducing the velocity $u$ of the particles, $t$ can be replaced by $\frac{l}{u}$, and we obtain the equation

$$
x_{1}=\frac{1}{2} \frac{F e}{m} \frac{l^{2}}{u^{2}} .
$$

After leaving the electric field the particles move along a tangent to the parabola, and can be shown to strike the screen at a point $P$, the distance $x_{2}$ of which from $O$ is given by

$$
x_{2}=\frac{F e}{m} \frac{l}{u^{2}}\left[d+\frac{l}{2}\right]
$$

where $d$ is the distance of the screen from the ends of the plates $A$ and $B$.

Since the cathode stream consists of negatively charged particles, it will behave as a conductor carrying a current in a direction opposite to that in which the particles travel. If therefore the rays pass through a magnetic field at right angles to the lines of magnetic force, they will be acted on by a force at right angles to their direction of motion and to the direction of the magnetic force. If $r$ is the radius of curvature of the path, the centrifugal force $\frac{m u^{2}}{r}$ at any point must be equal to the magnetic force $H e u$, where $H$ is the intensity of the magnetic field. Consequently,

$$
H e=\frac{m u}{r} \text {. }
$$

Hence it follows that $r$ is constant along the path, and that the rays are bent into a circle, provided that the velocity remains unaltered.

In order to use the same apparatus as that employed in the experiments on the electrostatic deflection, the radius of curvature must be so large that the deflection can be observed on the screen. In this case an expression can be found for $r$ in terms of the deflection, and equation (22) has then to be modified. If the displacement $y_{1}$ at the end of the magnetic field is small compared with the length $l$ of the field, it can easily be shown that

$$
y_{1}=\frac{H e l^{2}}{2 m u} \text {. }
$$


If the displacement is observed on a screen at a distance $d$ from the end of the magnetic field, the rays will strike the screen at a distance $y_{2}$ from $O$, such that

$$
y_{2}=\frac{H e}{m} \frac{l}{u}\left[d+\frac{l}{2}\right]
$$

From measurements of the electrostatic and magnetic deflections of the cathode rays, the values of $\frac{e}{m}$ and $u$ can be calculated by means of equations (21) and (24). The value of $\frac{e}{m}$ is $1.86 \times 10^{7}$ E.M. units and the velocity usually lies between $10^{9}$ and $10^{10} \mathrm{cms}$. per second.

The velocity of the cathode rays can also be determined by balancing the electric and magnetic deflections, so as to leave the rays undeviated. The electric and magnetic fields are applied simultaneously at right angles to each other, so as independently to produce equal and opposite deflections on the screen. In these circumstances $x_{2}=y_{2}$, and we obtain from equations (21) and (24) the relation,

$$
V=\frac{F}{H}
$$

Hence, if $F$ and $H$ are measured, their ratio gives the velocity of the rays. The quantities $F$ and $H$ must, of course, be expressed in the same system of units. 


\section{CHAPTER IV.}

THE ALPHA RAYS.

\section{$\$ 29$. Ionisation produced by different Areas of Uranium Oxide.}

Since the $\alpha$ rays can penetrate only a small fraction of a millimetre of a substance such as uranium oxide, it follows that the ionisation produced by a layer of the oxide is due only to the radiation coming from a thin surface film of the active material. Consequently, if the film is not extremely thin, its activity will be independent of the thickness of the layer and proportional to its area. This may be shown in the following way. Layers of finely powdered uranium oxide about one millimetre thick are placed in small circular metal dishes of about two, four and six centimetres diameter respectively. Care should be taken to make the thicknesses of the layers approximately equal and not much greater than one millimetre, otherwise a correction will have to be made for the $\beta$ radiation, which increases with the thickness. The activity of each dish is then measured, and will be found proportional to the area of the dish; but a slight deviation from proportionality may arise owing to the absorption of the $\alpha$ rays by the rim. Since the fraction of the radiation absorbed in this way depends approximately on the radius of the dish used, the influence of the rim will be relatively greater in the case of small dishes than with the larger ones.

$\$ 30$. Ionisation produced by different Thicknesses of Uranium Oxide.

It is difficult to prepare layers of uranium oxide sufficiently thin to permit $\alpha$ radiation to escape from the whole volume 
of the material. It can, however, be done by a method described by M‘Coy.* A glass ring, one end of which is ground flat; is pressed on to an aluminium foil about onetenth of a millimetre thick to make a liquid-tight joint. The uranium oxide is finely powdered in an agate mortar. A small quantity is taken and shaken up with a few cubic centimetres of chloroform or alcohol, and rapidly poured on to the aluminium. The uranium oxide in suspension soon settles down, and the liquid is allowed to evaporate off.

A number of different films are prepared and their thicknesses determined by weighing. The ionisation produced by the films increases at first proportionally to the thickness, and ultimately reaches a limit at about one hundredth of a millimetre. With this thickness the $\alpha$ rays from the lowest layer can just penetrate the film. The following table shows the results obtained with films of area 6 square centimetres :

Table showing the Variation with Thickness of the $a$-RaY Activity of Uranium Oxide.

\begin{tabular}{c|c|c}
\hline $\begin{array}{c}\text { Weight of Uranium } \\
\text { Oxide in milligrams. }\end{array}$ & $\begin{array}{c}\text { Activity in divisions } \\
\text { per minute. }\end{array}$ & $\begin{array}{c}\text { Activity per unit } \\
\text { weight. }\end{array}$ \\
\hline $2 \cdot 1$ & $0 \cdot 75$ & $\cdot 36$ \\
$2 \cdot 8$ & $0 \cdot 97$ & $\cdot 35$ \\
$10 \cdot 4$ & $3 \cdot 10$ & $\cdot 30$ \\
$14 \cdot 9$ & $3 \cdot 86$ & $\cdot 26$ \\
$36 \cdot 5$ & $7 \cdot 80$ & $\cdot 22$ \\
\hline
\end{tabular}

On account of the constancy of its activity, a uranium oxide film serves as a convenient arbitrary standard. $\uparrow$ To compare the activities of minerals and radio-active preparations with it, films of the materials are prepared as described above. The films are made so thin that their activities are proportional to their weights. The following table gives some results obtained with films of area 6 square centimetres :

* M'Coy, Phil. Mag. 11. 176, 1906.

$\uparrow$ A very thin film of uranium oxide gives an ionisation current of about $1 \cdot 2 \times 10^{-3}$ E.s. units per milligram of uranium. 
Comparison of $a$-Ray Activities of Different Substances.

\begin{tabular}{|c|c|c|c|}
\hline Substance. & $\begin{array}{l}\text { Weight of film } \\
\text { in milligrams. }\end{array}$ & $\begin{array}{c}\text { Activity in } \\
\text { divisions } \\
\text { per minute. }\end{array}$ & $\begin{array}{l}\text { Activity per } \\
\text { unit weight. }\end{array}$ \\
\hline Uranium oxide - & $2 \cdot 1$ & $0 \cdot 75$ & $0 \cdot 36$ \\
\hline Joachimsthal pitchblende - & $5 \cdot 27$ & $6 \cdot 24$ & $1 \cdot 18$ \\
\hline Thorium oxide (freshly prepared) & $4 \cdot 27$ & $2 \cdot 05$ & $0 \cdot 48$ \\
\hline Thorite $-\quad-\quad-\quad-\quad-$ & $5 \cdot 29$ & $1 \cdot 41$ & $0 \cdot 27$ \\
\hline
\end{tabular}

The method as described is obviously not applicable to substances which evolve emanation freely.

\section{$\S 31$. The Ranges of $\alpha$ Particles and their Absorption by Matter.*}

The $a$ particles produce very intense ionisation along their path in a gas, and thereby lose their kinetic energy with the result that, after traversing a few centimetres of gas at atmospheric pressure, their velocity falls below the value at which they can ionise. The -mechanism by which $a$ rays are absorbed is peculiar to this type of radiation; for practically none of the particles are stopped in their flight through matter until they have almost reached the extreme limit of their path. Towards the end of their path the velocity of the particles diminishes very rapidly; in fact the particles behave as if they were suddenly stopped after traversing a few centimetres of gas at atmospheric pressure. The extreme distance which the particles travel before being stopped is known as their range. For a particular product the ranges of all particles are the same; but particles of different origin have different ranges, which are thus characteristic of the substances from which the particles are expelled. The range, which depends on the density of the gas, varies inversely as its pressure and directly as its absolute temperature. The temperature and pressure at which the range is measured must therefore be specified. Different products emit $a$ rays, the ranges of which in air at $15^{\circ} \mathrm{C}$. and atmospheric pressure vary from $2.5 \mathrm{cms}$. in the case of uranium to $8.6 \mathrm{cms}$. for thorium $C$. The range

* Rutherford, Radio-activity, 1912, §66 et seq. 
of the $\alpha$ particles of any particular product is connected with the transformation constant of the product, and relations have been established in the uranium, thorium and actinium series connecting the ranges of the $\alpha$ particles with the lives of the products which emit them. In general those products whose lives are short emit $\alpha$ rays whose range is large and vice versa.

The distance in air which an $\alpha$ particle can travel can be reduced by interposing thin metal foils in the path of the rays, and the difference between the range of the particle with and without the foil interposed is called its stopping power.

The absorption of $\alpha$ particles by different metals can be easily studied in the case of polonium. This substance is suitable for the purpose, as it decays only slowly and thus forms a practically constant source of homogeneous $\alpha$ radiation. A polonium film can be prepared as described in $\$ 69$.

To carry out the experiment, the polonium film is put inside a circular dish with a shallow rim and placed inside an $\alpha$-ray electroscope. The foils to be used to absorb the radiation can be placed over the active matter so as to rest on the rim of the dish without touching the film. If this is not done, part of the polonium may be detached from the plate and contaminate the foils, which thus become useless for future experiments. The thickness of the absorbing layer of metal can be varied by increasing the number of foils covering the dish. The thickness of each foil is determined by weighing and measuring its area. Aluminium foils of thickness about $0.003 \mathrm{~mm}$. can be obtained and will be found suitable for the experiment. A curve should be plotted giving the relation of the ionisation produced in the electroscope and the thickness of the absorbing layer of aluminium, as shown in figure 26. It will be found that the ionisation gradually decreases with increasing thickness of aluminium and becomes zero when the thickness of the aluminium is $0.029 \mathrm{~mm}$. This distance is the range of the $\alpha$ particles from polonium in aluminium. 
It is impracticable to determine the range of the $\alpha$ particles in other metals in this way on account of the difficulty of obtaining foils of suitable thickness. A comparison of the stopping powers of different metal foils can, however, be carried out. The foil is placed over the active film and the ionisation determined. The thickness of aluminium, which

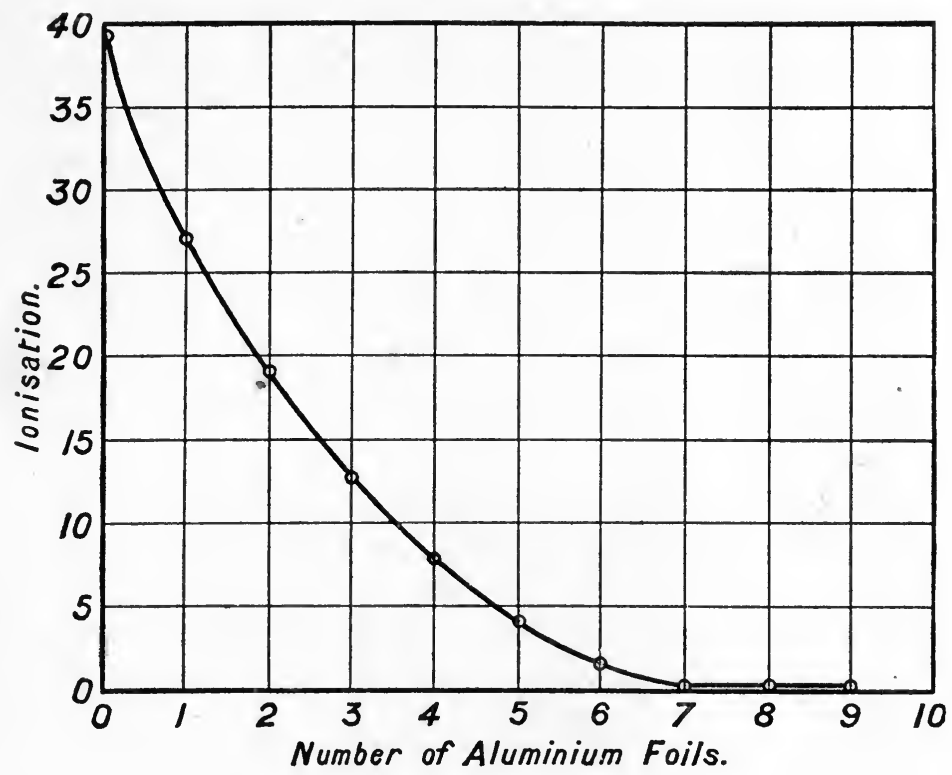

FI\%. 26.-Absorption of $a$ rays by aluminium.

produces the same reduction in ionisation as the foil, is taken from the absorption curve for aluminium, and this gives the stopping power of the foil in terms of aluminium. A more accurate method of determining stopping power is given in $\$ 36$.

$\$ 32$. The Variation of the Ionisation produced by an $\alpha$ Particle along its Path.

It has been shown by Bragg and Kleeman* that the ionising power of an a particle is approximately constant for the first part of its range, rises to a maximum just before the

* Bragg and Kleeman, Phil. Mag. 8. 726, 1904, and 10. 318, 1905. 
range of the particle is completed and ultimately falls rapidly to zero.

The variation of the ionisation produced by the $\alpha$ particles along their paths can be studied by means of the apparatus shown in figure 27 , which is a modification of that originally used by Bragg for the purpose. A plate $T$, which serves to carry the source of $\alpha$ rays, is fixed to the base of an $a$-ray electroscope so as to be at a distance of 1.5 or $2 \mathrm{cms}$. from the upper plate $A A$ of the condenser. The plate $A A$ is enclosed in a metal case $C B B C$ attached to the top of the lower chamber of the electroscope. The bottom of the case carries a grid consisting of a series of tubes fixed at right angles to $B B$, as shown in the figure. The tubes can be made by rolling aluminium or copper foils into cylinders about $1 \mathrm{~cm}$. long and

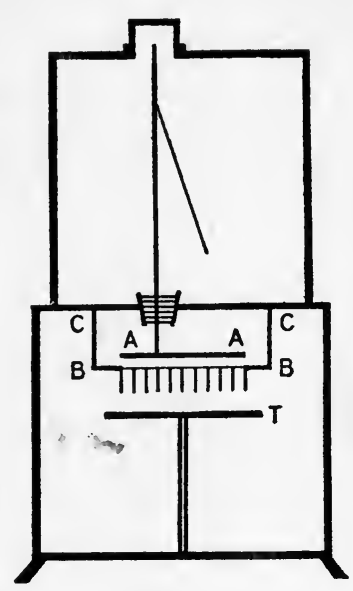

FIG. 27. about $5 \mathrm{mms}$. in diameter. The distance between $A A$ and $B B$ is about $3 \mathrm{mms}$.

The radiation from $T$ is emitted equally in all directions, but on account of the presence of the tubes it is only those rays which leave the active plate approximately at right angles which can reach the space between $A A$ and $B B$ and ionise the gas there. A very thin aluminium leaf covering the top of the grid prevents ions, produced inside the tubes, from being drawn into the ionisation chamber. The active deposit from thorium is suitable as a source of radiation on account of the long range of the $a$ particles emitted by it. A strongly active plate can be prepared as described in $\$ 44$. Since the active deposit decays with a period of $10 \cdot 6$ hours, corrections must be introduced for the loss of activity during an experiment (see Appendix II.). The ionisation between $A A$ and $B B$ is first measured with the source of radiation uncovered. Aluminium foils, each of thickness $0.003 \mathrm{~mm}$. and corresponding in stopping power to about $5 \mathrm{mms}$. of air, are 
then placed over the active material. A curve showing the ionisation with different numbers of foils superposed on the active film is given in figure 28. It will be seen that the curve shows two distinct maxima corresponding to the $\alpha$ particles emitted by thorium $C_{1}$ and thorium $C_{2}$ respectively. The residual activity left after complete absorption of the $\alpha$ rays is mainly due to $\beta$ radiation.

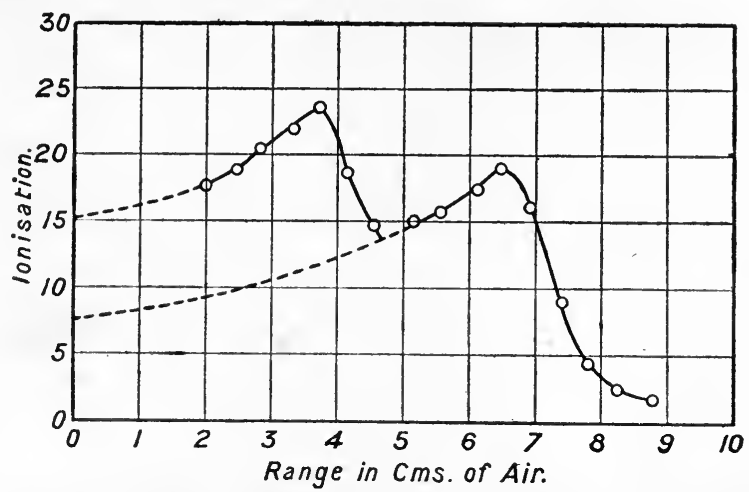

FIG. 28.-Ionisation produced by the $\alpha$ rays from thorium active deposit.

\section{$\$ 33$. Method of Counting Scintillations.}

It was first observed by Crookes that when radium is brought near a zinc sulphide screen, the phosphorescence produced is due to a number of independent flashes of light, which can easily be seen with the naked eye. It has since been shown that each flash of light observed on the screen is due to the impact of an $\alpha$ particle, and that almost every particle striking the screen is effective in producing a scintillation. The phenomenon may therefore be made use of not only to detect the presence of a particles, but also to determine quantitatively the number of particles emitted by the source of radiation ( $\$ 64)$. The method thus afforded of studying the rays is very direct, and may frequently be applied with advantage to investigate the $\alpha$ rays when other methods are inapplicable.

The observations are usually made with a zinc sulphide screen made by sprinkling the fluorescent salt on a thin glass plate. A good way of preparing such a screen is to 
cover a glass plate with a thin and uniform layer of a solution of Canada-balsam in xylol. The plate is allowed to stand until it has become nearly dry. Some phosphorescent zinc sulphide is then sprinkled over the surface and rubbed into the Canada-balsam.* Although the scintillations produced by bringing a source of $\alpha$ radiation near the zinc sulphide can be seen with the naked eye or a lens, it is best for quantitative work to use a low-power microscope specially arranged, so as to collect as much light as possible from the scintillations. For this purpose an objective of high light collecting power combined with a low-power eye-piece is used. The area of the field of view which can be observed with a microscope, the magnification of which is 70 , is about one square millimetre, which for most cases is sufficient. $\dagger$ For convenience of observation, a cork with a hole in the centre should be attached to the eye-piece; otherwise the ey is apt to move, with the result that some of the scintillations may escape observation. For the same reason it is advisable for the screen to be very faintly illuminated while counting. This is best done by a small electric lamp in series with an adjustable resistance. In absolute darkness it is very difficult to keep the eye fixed on the screen. The illumination of the screen should of course be so faint that the scintillations appear as distinct flashes on the screen. Before starting to make observations, it is necessary to remain in the dark for about 20 minutes, so as to allow the eyes to become accustomed to darkness. If this is not done, it is hardly possible to count the scintillations with certainty and accuracy. It is best to count for one or two minutes, and after resting the eye to count again for another two or three minutes, and so on. The number of scintillations observed in each period is noted and the time of observation recorded with a stop-watch; from these observations, the average number of $\alpha$ particles striking the screen per minute is determined.

* Svedberg, Zeitschr. für Phys. Chem. 74. 738, 1910.

† It has been found that Leitz objective No. 4, combined with eye-piece No. 0 giving a magnification 70, or objective No. 3, combined with eye-piece No. 0 giving a magnification 49 , are very suitable.

R.-A. 
Measurements can be made most accurately with about 40 scintillations per minute. With more than about 80 or less than about 10, the counting becomes troublesome and uncertain.

\section{$\$$ 34. Determination of the Range of a Particles by Scintil- lations.}

In order to determine the range of the $\alpha$ particles of a radio-active substance, a zinc sulphide screen is attached rigidly to the microscope at such a distance from the objective that it is perfectly in focus. The microscope, which is mounted on a graduated platform along which it can slide is brought close up to the source which emits the $\alpha$ rays. Scintillations will be observed on the screen, which should then be gradually withdrawn from the source. When a polonium film is used as source, it will be found that the scintillations cease almost suddenly when the distance from the film has been increased to $3.8 \mathrm{cms}$. This is particularly noticeable when the source is strong. The distance at which scintillations disappear can be measured on the scale upon which the microscope is mounted, and is the range of the $a$ particle from polonium. This method is applicable to other a-ray products, such as, for example, radium $C$, thorium $C$ and actinium $C$.

\section{$\S 35$. The sudden Stoppage of $a$ Particles at the End of their Range.}

It has been pointed out that, in passing through air, practically none of the $\alpha$ particles are stopped until within a few millimetres of the end of their range (\$31). This fact can be illustrated by counting the number of scintillations on a screen placed at different distances from a source of radiation. The experiment is, however, somewhat complicated by the fact that the number of $\alpha$ particles reaching the screen varies inversely as the square of the distance from the source. On this account it is practicable only to take observations over small distances, since, as the screen is withdrawn from the source, the number of scintillations soon becomes too small to be counted with precision. It is therefore more convenient to perform the experiment by keeping the screen at a fixed 
distance from the source and interposing metal foils, or by varying the pressure of the air between the source and screen. On interposing metal foils it will be found that the number of scintillations remains constant until the thickness of foils is increased to an amount sufficient almost to stop the radiation. On interposing more foils the number of scintillations then falls off rapidly to nought.

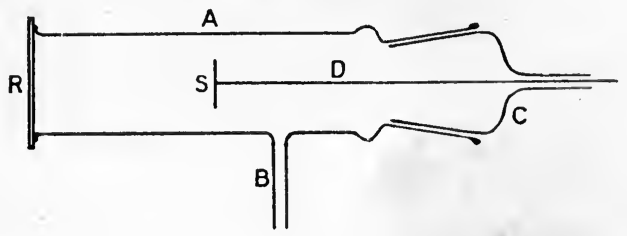

FIG. 29.

The experiment can also be carried out by means of the following apparatus. The source of radiation $S$ is attached to a $\operatorname{rod} D$ fixed to a glass stopper $C$, as shown in figure 29, and mounted some distance away from the zinc sulphide screen $R$, which is viewed through a microscope. The vessel

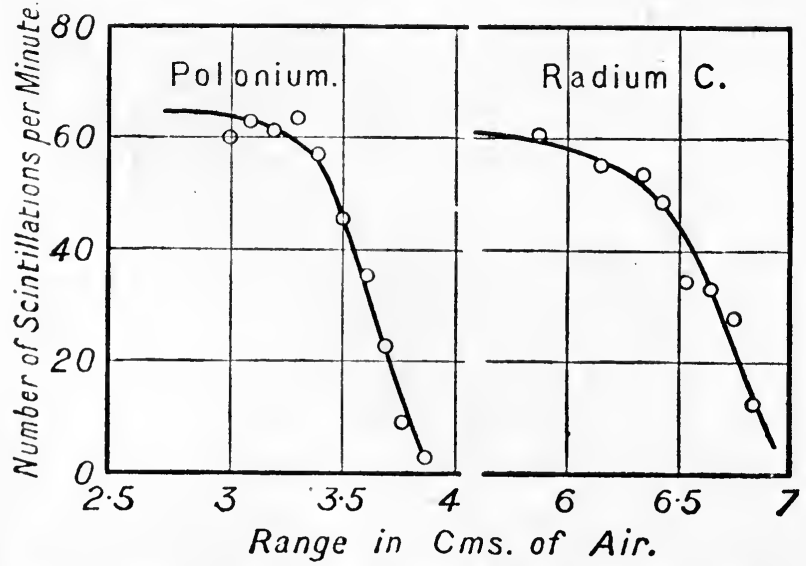

FIG. 30.-Diminution in the number of $a$ particles at the end of their range in air.

$A$ is exhausted through the side tube $B$. The number of scintillations appearing on the screen is counted at different air pressures. It will be found that the number of $\alpha$ particles reaching $R$ remains constant until a certain pressure is attained, when the number of scintillations rapidly diminishes. 
Since the stopping power of air is proportional to its density, the thickness of air at atmospheric pressure, equivalent to the air intervening between source and screen, can be calculated for each observation. Hence the range of the $a$ particles at normal pressure can be deduced. Typical curves showing the variation of the number of a particles near the end of their paths are shown in figure 30 . The curves refer to the cases of polonium and radium $C$ respectively.

The apparatus just described may also be used to determine the ranges of the $\alpha$ particles in different gases.

\section{$\$ 36$. The Stopping Power of Metal Foils.*}

The stopping power of a metal foil has already been defined, and a method has been given of determining this quantity (\$31). Stopping power is an atomic property. For a single atom it is proportional to the square root of its mass, and for a molecule it is the sum of the square roots of the masses of the atoms composing it. $\dagger$ If therefore the number of atoms contained in two metal foils of equal area are to each other in the inverse ratio of the square roots of their atomic masses, the two films have the same stopping powers.

The stopping power of a substance depends on the speed of the particles. For substances of about the same atomic weight as air the stopping power is nearly independent of the speed; but for the heavier molecules the stopping power somewhat decreases as the speed of the $\alpha$ particles passing through it diminishes. +

Stopping powers can be accurately measured by the method of scintillations, which is therefore suitable for testing the above laws. A strong source of $\alpha$ radiation, such as a plate coated with polonium, is used to determine the range of the $\alpha$ particles. A metal foil is inserted in the path of the rays, and the point at which the scintillations just disappear is again determined. The change in position of the microscope, with and without the foil, measures its stopping

* Rutherford, Radio-activity, 1912, §71.

† Bragg and Kleeman, Phil. Mag. 10. 318, 1905.

+Taylor, Plil. Mag. 18. 604, 1909. 
power. The following figures given by Bragg and Kleeman illustrate the degree of accuracy with which the square root law holds :

Relative Stopping Powers for a Particles.

\begin{tabular}{lr|c|c}
\hline \multicolumn{1}{r|}{ Substance. } & . & $\begin{array}{c}\text { Stopping Power of } \\
\text { Atom or Molecule. }\end{array}$ & $\sqrt{\frac{\text { Atomic weight of substance }}{\text { Atomic weight of air. }}}$ \\
\hline Hydrogen & - & $\cdot 246$ & $\cdot 265$ \\
Air - & - & 1 & 1 \\
Aluminium - & $1 \cdot 53$ & $1 \cdot 38$ \\
Copper - & - & $2 \cdot 42$ & $2 \cdot 1$ \\
Silver - & - & $3 \cdot 11$ & $2 \cdot 75$ \\
Tin - & - & $3 \cdot 42$ & $2 \cdot 88$ \\
Platinum & - & $4 \cdot 12$ & $3 \cdot 7$ \\
Gold - & - & $4 \cdot 45$ & $3 \cdot 7$ \\
\hline
\end{tabular}

The variation of stopping power with the speed of the $\alpha$ particles can be measured by interposing the foil at different distances from the source. It will be found that the stopping power is almost independent of the velocity, as mentioned above.

$\$ 37$. The Laws of Probability as applied to the Emission of $\alpha$ Particles. $\dagger$

If $\alpha$ particles are allowed to fall on a phosphorescent screen, the number of scintillations appearing in a fixed time varies even though the source of radiation is constant. Thus, if a source is said to emit a certain number of $\alpha$ particles per minute, the statement implies that the particular number is emitted on the average per minute, but the number actually generated in different minutes may vary between wide limits. The phenomenon can be studied by the method of counting scintillations, which affords an interesting means of testing the laws of probability as applied to atomic changes.

If, from a constant source of $\alpha$ radiation the number of scintillations in successive short intervals of time is counted, it will be found that the observations show variations which may

* The atomic weight of the hypothetical atom of air is taken as 14.4 .

+ Rutherford, Radio-activity, 1912, §75. 
be compared with the fluctuations predicted by the theory of probability. Bateman* has shown that if $a$ is the true average number of particles received by a phosphorescent screen in a given time, then the probability $p$, that $n$ particles will be observed in that time interval, is given by

$$
p=\frac{a^{n}}{n !} e^{-a}
$$

where $n$ may be given the values $0,1,2,3, \ldots$.

To test the equation, a plate coated with polonium is placed at such a distance from a zinc-sulphide screen that about 50 scintillations appear on it per minute. The number of scintillations appearing in equal successive intervals of about 15 seconds is then determined. Since the experiment must be carried out in the dark, the assistance of a second observer is necessary, or else an automatic device must be employed for recording the scintillations. This can be done by registering the appearance of each scintillation on a tape travelling at a known constant rate, and subsequently counting the number of scintillations occurring in successive intervals of 15 seconds. The observations must be continued for a large number of intervals, say 500, from which the average number of scintillations can be deduced. The observations are then classified into groups indicating the number of occasions on which the time interval of 15 seconds is characterised by scintillations appearing once, twice, three times and so on.

From the average number $a$ of scintillations appearing in the selected time interval, the value of $p$ for different values of $n$ can be calculated and a theoretical curve plotted, as shown in figure 31. In the example given, $a=3.8$ for an interval of 15 seconds. The points marked on the curve are experimental. $\dagger$ The degree of agreement between the experimental and theoretical curves will of course depend on the number of intervals of time over which the observations extend. Thus, the longer the experiment is continued the better will be the agreement.

* Bateman, Phil. Mag. 20. 704, 1910.

† Rutherford and Geiger, Phil. Mag. 20. 698, 1910. 
The phenomenon may be looked at from a somewhat different point of view.* Instead of considering the probability of any number of scintillations occurring in a given time, the probability of' a definite time interval elapsing

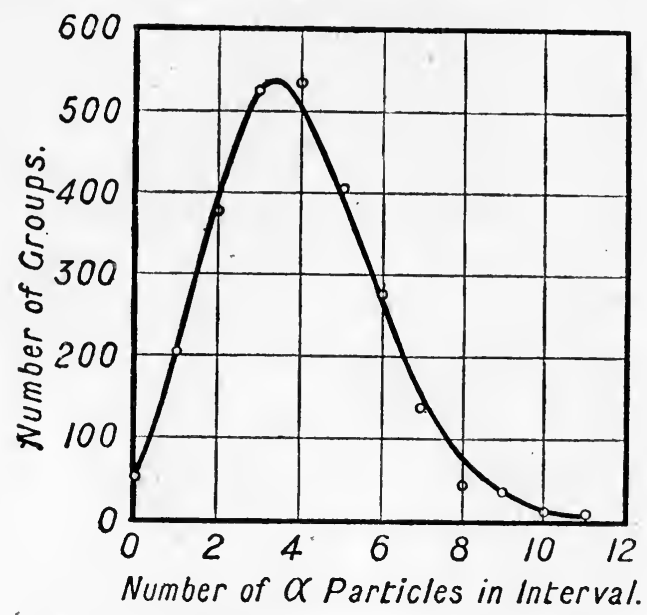

FiG. 31.-Probability curve of emission of $a$ particles.

between two successive scintillations may be calculated and compared with experiment. If $\frac{1}{a}$ is the average time interval between successive scintillations, it can be shown that the probability of the occurrence of an interval greater than $t$ and less than $t+\delta t$ is given by

$$
p=a e^{-a t} \delta t .
$$

It follows from this equation that the probability of a short interval is always greater than that of a long one.

* Marsden and Barratt, Proc. Phys. Soc. 23. 367, 1911. 
$[\mathrm{CH}$.

\section{CHAPTER V.}

\section{THE BETA AND GAMMA RAYS.}

\section{$\$ 38$. The Absorption of $\beta$ Rays by Matter.*}

In order to study the absorption of $\beta$ rays by matter, the active plate to be examined is placed at such a distance from a $\beta$-ray electroscope that it gives an ionisation current of about 40 divisions per minute. Thin sheets of the absorbing material are interposed in the path of the rays and the activity measured through successive thicknesses. The absorbing sheets should be placed close to the electroscope to avoid complications due to scattering (\$40).

The $\beta$ Radiation from Uranium Oxide. A dish of about $6 \mathrm{cms}$. diameter containing about 50 grams of uranium oxide is placed in position near the electroscope. Different numbers of aluminium foils, about $0.1 \mathrm{~mm}$. thick, are interposed and the ionisations produced in the electroscope in each case observed. Since the foils are usually not quite uniform in thickness, it is best to determine the average thickness of each one by weighing. It will be found that the ionisation gradually diminishes as the thickness of the absorbing layer increases and ultimately tends to a small constant limit. This residual activity, which is undiminished by increasing the absorbing layer, is due to the $\gamma$ rays. In order to find the ionisation produced by the $\beta$ rays alone, it is necessary to subtract the ionisation due to the $\gamma$ rays from each of the values of the ionisation obtained with different absorbing layers of aluminium. The values so obtained for the $\beta$ radiation entering the electroscope are plotted against the thickness of the aluminium interposed (fig. 32, $B$ ). It will be found that the ionisation decreases exponentially

\footnotetext{
* Rutherford, Radio-activity, 1912, § 82.
} 
with the thickness of the aluminium, as can be seen from the straight line obtained by plotting the logarithm of the activity against the thickness (fig. $32, B^{\prime}$.)

If $I_{0}$ is the ionisation produced in the electroscope when no aluminium is interposed and $I$ is the ionisation when the rays pass through a thickness $d$ of aluminium, then

$$
I=I_{0} e^{-\mu d} \text {. }
$$

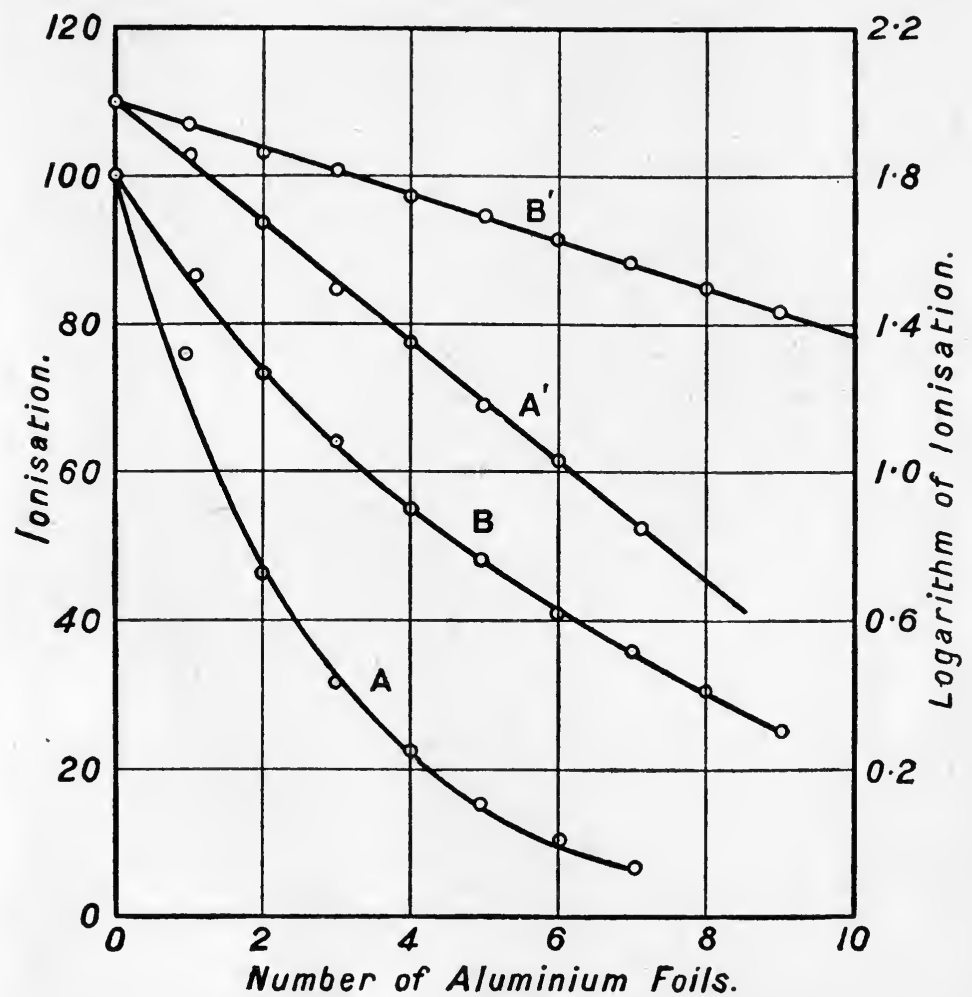

FiG. 32.-Absorption of $\beta$ rays from uranium and radium $E$ by aluminium.

The constant $\mu$ is known as the coefficient of absorption of the $\beta$ rays by aluminium. Its value can be deduced from curve $A^{\prime}$ for

$$
\mu=\frac{1}{d} \log _{e} \frac{I_{0}}{I}=\frac{2 \cdot 302}{d}\left(\log _{10} I_{0}-\log _{10} I\right) .
$$

The experiment can be repeated with foils of other metals. 
The absorption coefficients of the $\beta$ rays from uranium $X$ in aluminium, copper and lead are $15 \mathrm{~cm}^{-1}, 66 \mathrm{~cm}^{-1}$ and $103 \mathrm{~cm}^{-1}$ respectively.

The $\beta$ Radiation from Radium $\boldsymbol{E}$ and the Active Deposits. The experiment should be repeated with other radio-active products emitting $\beta$ radiation, as for instance radium $E$. With this product the rays are more easily absorbed than with uranium oxide, and are said to be softer (fig. $32, A$ and $A^{\prime}$ ). In order to obtain an absorption curve, thinner layers of absorbing material should be used than when working with uranium oxide. The results will in general be found to be similar to those obtained with uranium oxide.

The experiment can also be made with the active deposit from actinium, but in this case the measurements are complicated by the decay of the active matter during an experiment. The rate of decay is controlled by the period of actinium $B$, which is 36.3 minutes, so that corrections can be introduced for the decay of activity during the experiment if the time of each observation is recorded (Appendix II.).

In the three cases just considered, the absorption curve is exponential, showing that each successive layer of material interposed produces the same percentage reduction in the radiation remaining after traversing the previous layers. It might be thought that an exponential law of absorption is a proof that all the rays are of the same velocity. It has, however, been shown by Wilson* that an exponential law of absorption cannot be taken as a test of the homogeneity of the rays. Moreover, photographic experiments on the deflection in a magnetic field show that rays which obey an exponential law of absorption can be split up into groups of different velocities. $\dagger$ When the differences of velocity of the groups are large, the law of absorption is no longer exponential, as may be exemplified by the $\beta$ radiation from the active deposits of radium and thorium. In both these cases the radiation is very complex; with the active deposit from radium, the radiation is made up of

${ }^{*}$ W. Wilson, Proc. Roy. Soc. A. 82. 612, 1909.

† Von Baeyer, Hahn and Meitner, Phys. Zeitschr. 12. 273 and 1099, 1911. 
rays from radium $C$ and rays from radium $B$, which are for the most part much softer; with the active deposit of thorium, thorium $B$ gives soft rays and thorium $C$ and thorium $D$ hard ones. Experiments should be made to test the law of absorption in these two cases. The results obtained with the active deposit of thorium are shown in figure 33, from which it will be seen that the rate of diminution of activity is at first much greater than afterwards. This indicates the presence of soft radiation. A table is given showing the absorption in aluminium for different sources of radiation.

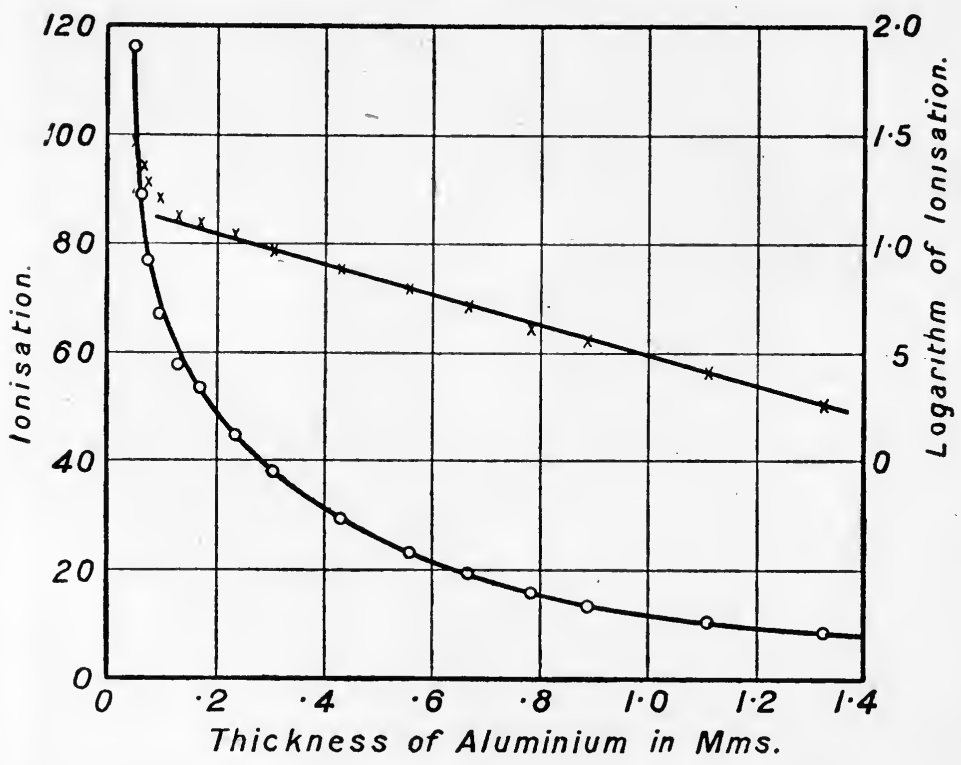

FIG. 33.-Absorption of $\beta$ rays from thorium active deposit by aluminium.

Absorption of $\beta$ Rays in Aluminium.

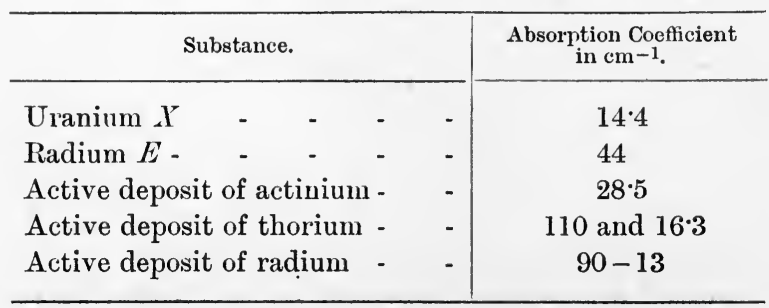


$\$ 39$. The $\beta$ Radiation from different Thicknesses of Uranium.

To investigate the variation of the $\beta$ radiation emitted by layers of uranium oxide of different thicknesses, a dish of diameter about $6 \mathrm{cms}$. is covered with a thin uniform layer of uranium oxide. The dish is placed in front of the aluminium window of a $\beta$-ray electroscope and the ionisation measured. The experiment is repeated with layers of

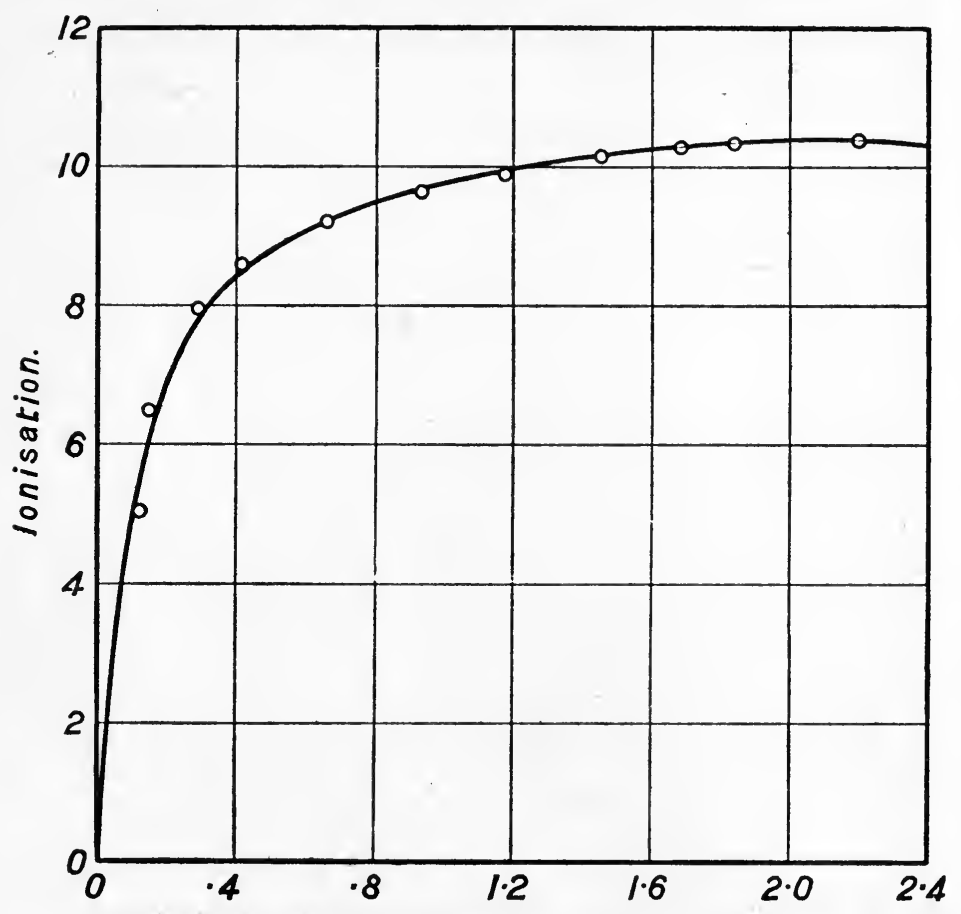

Thickness of Layers of Uranium Oxide in Crms.per Sq. Cm.

FIG. 34.-Variation of intensity of $\beta$ radiation with thickness of layer of uranium oxide.

uranium oxide of different thicknesses. For thin layers, the ionisation is proportional to the thickness, but increases less rapidly as the thickness becomes greater, ultimately reaching a limit when the thickness is so great that the radiation from the lower layers of uranium oxide is completely absorbed by the active material above it. The thickness of the layers is best found by weighing, and can be expressed in grams of 
uranium oxide per square centimetre. The measurement should be made for thicknesses ranging from $0 \cdot 1$ gram per square centimetre to 2 or 3 grams per square centimetre. The results of a typical experiment are shown in figure 34 .

From the results obtained in the experiment, the absorption coefficient of the $\beta$ rays from uranium oxide by the active material itself can readily be calculated. Let $\delta x$ be the thickness of a thin layer situated within the active material at a distance $x$ from the surface. Then if $\mu$ is the coefficient of absorption of the active material for the $\beta$ rays, the ionisation in the electroscope, due to the thin layer considered, will be given by

$$
\delta I=I_{0} e^{-\mu x} \delta x,
$$

where $I_{0} \delta x$ denotes the ionisation produced in the electroscope by a layer of thickness $\delta x$ situated at the surface of the active material.

The total ionisation produced in the electroscope by a layer of finite thickness $d$ will be given by the expression

$$
I_{d}=I_{0} \int_{0}^{d} e^{-\mu x} d x=\frac{I_{0}}{\mu}\left(1-e^{-\mu l}\right) .
$$

As $d$ increases, this quantity approaches the limit $\frac{I_{0}}{\mu}$. Consequently, $I_{d}=I_{\infty}\left(1-e^{-\mu l}\right)$, where $I_{\infty}$ is the ionisation produced by a thick layer of material. The value of $I_{d}$ and $I_{\infty}$ can be obtained from the experiment and the coefficient of absorption of the uranium oxide for the $\beta$ rays thus deduced.

\section{$\$ 40$. The Scattering of $\beta$ Rays by Matter.*}

When $\alpha$ or $\beta$ rays strike a plate, the particles comprising the radiation are in general deflected from their paths so as to emerge as a scattered beam, even though the incident rays are confined between definite limits. This is explained by supposing that the particles passing into the plate actually penetrate the atoms, and in their passage through them are subjected to very strong electric fields. The particles are thereby deflected from their course by varying amounts, and may even be turned through angles greater than a right angle, being thus diffusely reflected so as to emerge on the

* Rutherford, Radio-activity, 1912, § 83 et seq. and $\$ 86$ et sel . 
same side of the plate as that on which they impinged. The effect is much more marked with $\beta$ than with $\alpha$ rays.

Variation of Reflection with Material of Reflector. The phenomenon may be studied by means of the arrangement shown in figure 35 . It is best to employ radium $E$ as the source of radiation, or, if radium $E$ is not available, the active deposit of thorium can be used. The active matter must be deposited on an aluminium foil $A$ of thickness not greater than $0.01 \mathrm{~mm}$. The active foil is placed just below the aluminium window $W$ of a $\beta$-ray electroscope, and is supported in such a way that there is only air immediately below the foil. The supports must be small and the whole system removed as far as possible from surrounding objects, so that

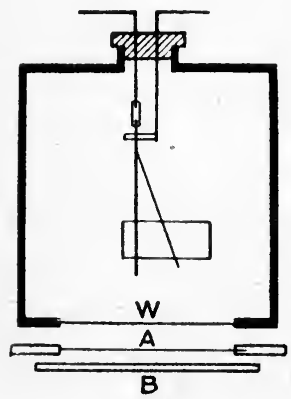

FIc. 35. no radiation can be reflected by them into the electroscope. In these circumstances the ionisation in the electroscope is produced entirely by the radiation entering it directly from the active body, except for a small quantity of scattered radiation from the foil on which the active material is deposited. The ionisation in the electroscope is measured and a lead plate $B$, which is used as a reflector, is then placed immediately below the foil and the ionisation again observed. It will be found on inserting the plate that the ionisation is increased, indicating that some of the rays proceeding in a downward direction, which previously did not enter the electroscope, are now reflected back into it and contribute to the ionisation produced. The difference between the ionisation with and without the lead plate gives a measure of the radiation reflected by the lead. If $I_{1}$ and $I_{2}$ are the ionisations with air and lead respectively. below the source, $100 \frac{I_{2}-I_{1}}{I_{1}}$ gives the percentage reflection
from lead.

The experiment should be repeated with plates of other substances such as carbon, aluminium, copper and tin, and a curve plótted showing the relation between the increase 
produced in the ionisation and the atomic weight of the reflecting material.

Some typical results obtained by Kovarik* with radium $E$ and actinium $D$ as sources of radiation are tabulated below.

Reflection of $\beta$ Rays.

\begin{tabular}{l|c|c|c}
\hline \multirow{2}{*}{ Reflector. } & Atomic Weight. & \multicolumn{2}{|c}{ Percentage Reflection. } \\
\cline { 2 - 3 } & Radium $E$. & Actinium $D$. \\
\hline $\mathrm{Bi}$ & $208 \cdot 5$ & $70 \cdot 9$ & $81 \cdot 0$ \\
$\mathrm{~Pb}$ & $206 \cdot 9$ & $70 \cdot 2$ & $80 \cdot 0$ \\
$\mathrm{Au}$ & $197 \cdot 2$ & $67 \cdot 8$ & $78 \cdot 7$ \\
$\mathrm{Pt}$ & $194 \cdot 8$ & $67 \cdot 7$ & $77 \cdot 6$ \\
$\mathrm{Sn}$ & $119 \cdot 0$ & $62 \cdot 5$ & $69 \cdot 7$ \\
$\mathrm{Ag}$ & $107 \cdot 9$ & $57 \cdot 4$ & $63 \cdot 5$ \\
$\mathrm{Zn}$ & $65 \cdot 4$ & $45 \cdot 5$ & $52 \cdot 6$ \\
$\mathrm{Cu}$ & $63 \cdot 6$ & $44 \cdot 7$ & $51 \cdot 9$ \\
$\mathrm{Ni}$ & $58 \cdot 7$ & $43 \cdot 5$ & $48 \cdot 0$ \\
$\mathrm{Fe}$ & $55 \cdot 9$ & $41 \cdot 2$ & $47 \cdot 1$ \\
$\mathrm{~S}$ & $32 \cdot 1$ & $32 \cdot 1$ & $40 \cdot 1$ \\
$\mathrm{Al}$ & $27 \cdot 1$ & $30 \cdot 0$ & $38 \cdot 3$ \\
$\mathrm{C}$ & $12 \cdot 0$ & $17 \cdot 1$ & $27 \cdot 4$ \\
\hline
\end{tabular}

Variation of Reflection with Thickness of Reflector. It is interesting to investigate the effect of varying the thickness of the reflector in the above experiment. This can be done with thin aluminium foils of thickness about $0 .(003 \mathrm{~mm}$., or preferably with substances of higher atomic weight, if available in thin enough sheets. The foils are placed under the active foil in gradually increasing numbers, and the ionisation in the electroscope in each case observed. It will be found that the radiation reflected into the electroscope at first increases as the number of foils of aluminium is increased, but soon reaches a limit for greater thicknesses. It thus appears that the phenomenon of reflection is not a surface effect, but depends on the thickness of the reflector. The $\beta$ particles, impinging on the reflector, penetrate into the material and are deflected from their course inside the matter at appreciable depths from the surface. The deflected

* Kovarik, Phil. Mag. 20. 849, 1910. 
particles then again penetrate the medium, and, after finding their way to the surface of the reflector, pass into the electroscope. The amount of reflected radiation entering the electroscope thus increases until the reflector is so thick that the particles deflected in the lower layers of the matter are practically all absorbed before again reaching the surface.

The part which scattering may play in certain radio-active measurements has recently been demonstrated in a striking way by Kovarik.* When making measurements on the absorption of thin metal sheets, the radio-active matter is usually placed some distance below the $\beta$-ray electroscope, and the absorbing screens near the electroscope. In this case any scattering which takes place will be without effect on the radiation entering the electroscope, for any radiation which emerges from the foil on the side of the electroscope is bound to enter it. If, however, the thin absorbing foil is placed directly over the radio-active film, and this is placed some distance below the electroscope, the case is different. Before inserting the foil, only those rays subtended at the source by the aperture of the electroscope contribute to the ionisation. On interposing the foil, some of these rays will be scattered away; but the diminution in the ionisation thus produced may be counteracted or even outweighed by radiation, which, though previously not entering the electroscope, is now scattered into it. It may thus happen that when very thin layers are interposed the ionisation may actually be increased.

To carry out an experiment, a strong preparation of radium $E$ is placed about 15 or 20 centimetres below a $\beta$-ray electroscope. Thin aluminium foils of thickness $0.003 \mathrm{~mm}$. are superposed directly over the radio-active matter, and the ionisation determined in each case.

Some typical results obtained by Kovarik with aluminium and tin foils are shown in figure 36. The points marked with circles refer to aluminium and those marked with crosses to tin.

When determining the absorption of $\beta$ rays, the effect * Kovarik, loc. cit. 
of scattering can be eliminated by depositing the active material on an aluminium leaf and placing the same number of foils below the active material as are placed above to absorb the rays. The radiation which is scattered away from the electroscope by the foils on one side of the active matter is counterbalanced by the radiation scattered into the electroscope by the foils on the other side. This precaution should be adopted for determining absorption coefficients accurately.

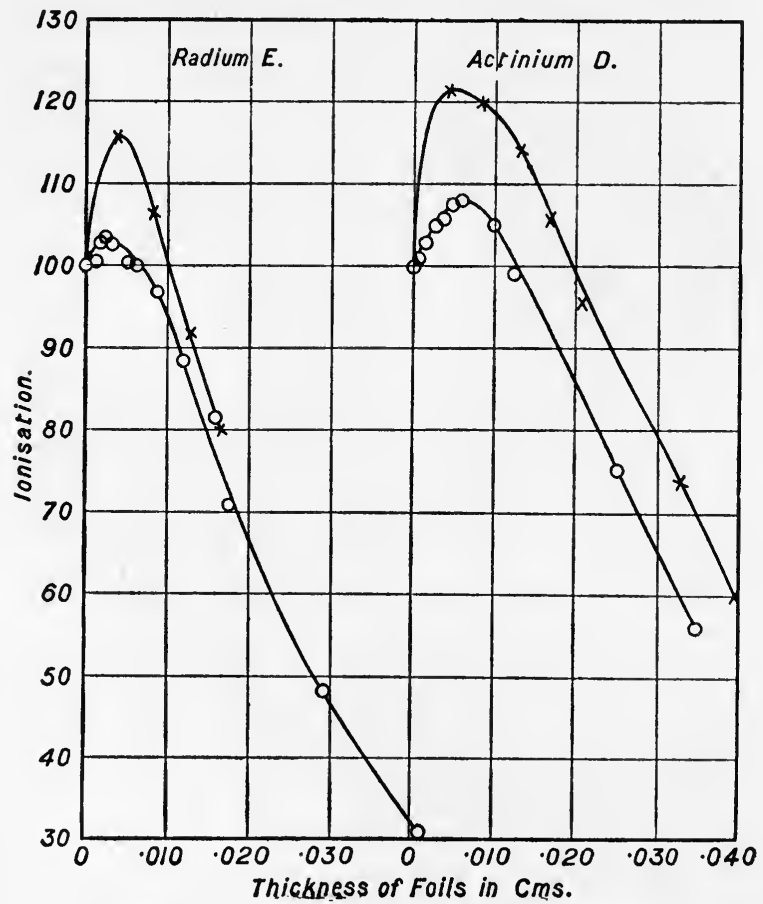

Fia. 36.-Influence of scattering on absorption curves for $\beta$ rays.

\section{$\$ 41$. The Absorption of the $\gamma$ Rays by Matter.*}

Since the $\gamma$ rays are capable of penetrating great thicknesses of matter, it is possible to use for their investigation a radio-active source contained in a closed vessel. About one milligram of radium sealed in a glass tube is a suitable amount to use for experiments on $\gamma$ rays, but in case this

* Rutherford, Radio-activity, 1912, §97 et seq.

R.-A. 
quantity is not available, it is possible with care to work with even so small a quantity as $0.1 \mathrm{mg}^{*}{ }^{*}$ In order to investigate the $\gamma$ rays, all $\beta$ rays must be prevented from entering the electroscope used for the measurements. This is done by using an electroscope made of lead two or three millimetres thick (\$16).

In order to determine the absorption of the rays by lead, screens of thickness from 2 to 3 millimetres are interposed between the source of radiation and the electroscope. As the number of screens is increased, the ionisation in the electroscope

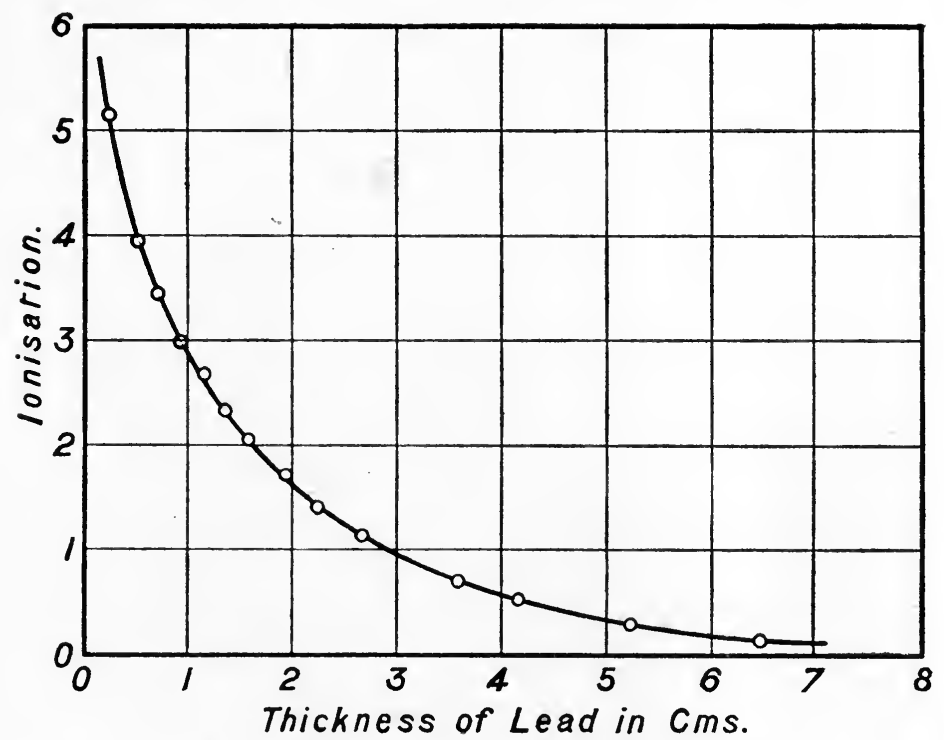

FIG. 37.-Absorption of the $\gamma$ rays of radium by lead.

gradually decreases, and a curve can be plotted showing the relation between the ionisation and the thickness of the absorbing layer of lead (fig. 37). It will be found that the curve is nearly exponential.

The experiment should be repeated with other materials such as copper, zinc, iron and brick, and the absorption coefficients for each calculated in the same way as explained

* Care must be taken that the tube is hermetically sealed; otherwise the emanation will escape and render apparatus in the neighbourhood radio-active. 
for the $\beta$ rays (\$38). The absorption coefficients $\mu$ will be found to be approximately proportional to the density $D$ of the absorbing material.* Some typical results are shown in the table, but it must be remembered that the values of the absorption coefficients depend to some extent on experimental conditions.

Absorption of $\gamma$ Rays from Radium.

\begin{tabular}{llll|l|l}
\hline \multicolumn{3}{c|}{ Substance. } & & $\mu$. & $100 \mu / D$. \\
\cline { 1 - 1 } Lead - & - & - & - & $0 \cdot 52$ & $4 \cdot 5$ \\
Copper & - & - & - & $\cdot 38$ & $4 \cdot 3$ \\
Brass & - & - & - & $\cdot 36$ & $4 \cdot 3$ \\
Iron - - & - & - & $\cdot 32$ & $4 \cdot 2$ \\
Tin - - & - & - & $\cdot 31$ & $4 \cdot 3$ \\
Zinc - - & - & - & $\cdot 31$ & $4 \cdot 3$ \\
Aluminium & - & - & $\cdot 12$ & $4 \cdot 4$ \\
Sulphur - & - & - & $\cdot 09$ & $5 \cdot 1$ \\
Paraffin wax & - & - & $\cdot 0047$ & $5 \cdot 2$ \\
\hline
\end{tabular}

$\$ 42$. Comparison of Absorptions of $\alpha, \beta$ and $\gamma$ Rays by Matter.

It is interesting at this stage to compare approximately the absorptions of the $\alpha, \beta$ and $\gamma$ rays by matter by finding the thickness of an absorbing material necessary to cut down each type of radiation to half its value. For this purpose paper serves very well as the absorbing medium. The measurements with $\alpha$ rays must be made in an $\alpha$-ray electroscope, and a source of uncovered active deposit from radium or thorium is used. The quantity of paper required to cut the activity down to half can be found by interposing screens of thin tissue paper (cigarette paper). For the $\beta$ rays the same source may be used, and ordinary sheets of note paper interposed to absorb the rays. For the $\gamma$ rays much greater thicknesses of the absorbing material are required, and books may be interposed between the source of radiation and the electroscope until the radiation is cut down to a determined fraction, from which the thickness to reduce the radiation to half value can be calculated.

* Russell and Soddy, Phil. Mag. 21. 130, 1911. 
The quantity of paper necessary to absorb half of each type of radiation will be seen from the following approximate numbers obtained with thorium active deposit:

$\alpha$ rays 0.003 centimetres,
$\beta$ rays 0.2
$\gamma$ rays 15.0

\section{\$43. Excited Radiation produced by $\gamma$ Rays.*}

When $\gamma$ rays strike a plate scattering takes place just as in the case of $\beta$ rays; but in addition to this effect $\beta$ rays are radiated from the plate struck by the $\gamma$ rays. The new type of rays thus produced is spoken of as excited radiation. The $\beta$ radiation comes out on both sides of the plate. That emitted on the side at which the $\gamma$ rays enter is called the "incidence" radiation, and that emitted on the other side is called the "emergence" radiation. The incidence and emergence radiations are different in amounts and have different velocities, and this dissimilarity is more marked with some

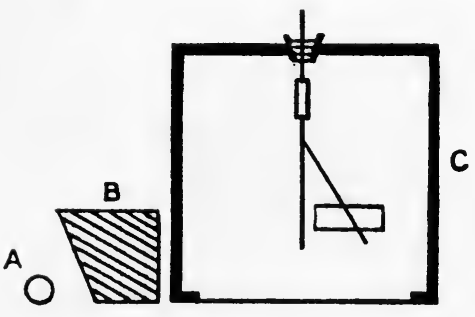

FIa. 38. substances than with others. In this paragraph only the incidence radiation is considered.

The existence of this radiation can be demonstrated and investigated by means of the arrangement shown in figure 38. $A$ is a source of radiation consisting of at least one milligram of radium, enclosed in a case thick enough to absorb all the $\beta$ rays. It is placed close to a $\beta$-ray electroscope $C$. A lead block $B$, not less than four centimetres thick, is interposed between the radium and the electroscope in such a way as to prevent the greater part of the $\gamma$ radiation from entering the electroscope directly. The whole arrangement should be mounted on a light wooden stand to avoid the entry into the electroscope of any appreciable excited radiation from external objects. The magnitude * Rutherford, Radio-activity, 1912, § 101. 
of the ionisation in the electroscope due to the direct effect from the radium must first be carefully measured. A thick lead plate $D$, of large area, is then placed below the electroscope as shown in the figure, so that some of the $\gamma$ rays from the radium can impinge upon it. 'The ionisation will increase mainly on account of the excited $\beta$ rays produced by the lead, and the difference between the ionisation with and without the plate $D$ in position gives a measure of the secondary radiation produced.

Nature of Excited Radiation. In order to show that the extra ionisation in the electroscope is in the main due to excited $\beta$ rays and not to scattering of the $\gamma$ rays by the plate, it is necessary to determine the absorption coefficient of the radiation. This can be done by repeating observations with thin sheets of aluminium or other absorbing material placed directly under the electroscope, so that the excited rays have to pass through them before entering the electroscope. By making a series of observations with different numbers of foils, the absorption coefficient of the rays can be found. The value of the absorption coefficient for aluminium is very nearly $20 \mathrm{~cm}^{-1}$, and is almost independent of the nature of the reflector. By increasing the number of absorbing foils, it will be found that the radiation cannot be completely absorbed, but that about $20 \%$ of the total radiation is of a penetrating character consisting of scattered $\gamma$ rays.

The excited radiation emerging in different directions can be studied by allowing a confined beam to fall normally on a plate, and observing the radiation emerging at different angles. Experiments of this kind, however, require considerable quantities of radium.

Variation of Excited Radiation with Reflecting Material. A further experiment should be made with thick plates of different materials placed at $D$, and the excited radiation produced in each case measured. In this way the excited radiation produced by different metals can be compared.

As will be seen from the following table, the excited radiation increases with the density of the reflector; but no 
[CH.

very definite relation between these two quantities is yet known. The amounts of excited radiation from different materials are tabulated below. The numbers are taken from a table given by Eve.*

Amount of Excited Radiation from Different Materials.

\begin{tabular}{lrr||c|c}
\hline \multicolumn{2}{c|}{ Substancc. } & Density. & $\begin{array}{c}\text { “Incidence" } \\
\text { Radiation. }\end{array}$ \\
\cline { 1 - 2 } Lead - - & - & - & $11 \cdot 4$ & 141 \\
Copper - & - & - & $8 \cdot 8$ & 79 \\
Iron - - & - & - & $7 \cdot 8$ & 75 \\
Aluminium & - & - & $2 \cdot 6$ & 42 \\
Paper - & - & - & -4 (about) & 20 \\
\hline
\end{tabular}

Variation of Excited Radiation with Thickness of Reflector. As in the case of the scattering of $\beta$ rays, the effect is one of volume and not of surface, so that the excited radiation depends on the thickness of the reflector used. The experimental investigation of this point can be conveniently made with tin or aluminium foils. By placing different numbers of foils at $D$, the variation of the excited radiation with the thickness can be studied. A thickness of $0.1 \mathrm{~mm}$. of tin or $0.5 \mathrm{~mm}$. of aluminium gives about half the maximum excited radiation.

* Eve, Phil. Mag. 8. 669, 1904. 


\section{CHAPTER VI.}

\section{THE ACTIVE DEPOSITS AND RADIO-ACTIVE RECOIL.}

$\$ 44$. The Distribution of the Active Deposits in an Electric Field and the Method of concentrating them on Surfaces.*

Radium, thorium and actinium give off radio-active gases called emanations, which in decaying form groups of successive solid radio-active products known as the active deposits. When the emanations are allowed to decay in an electric field, it is found that most of the active deposit formed is drawn to the cathode, and in this way can be concentrated on a surface. The experimental arrangement adopted to collect the deposit is slightly different for radium on the one hand and thorium and actinium on the other hand.

Thorium and Actinium Active Deposits. To render a wire $W$ active, it is connected to the rod $N$ passing through the ebonite stopper $S$, which fits into the brass tube $T$ (fig. 39). The thorium or actinium preparation is contained in the dish $D$ placed in the tube $T$. The tube is connected to the positive pole of a battery giving about 100 volts and the rod $N$ to the negative pole through a water resistance to prevent the possibility of short-circuiting the battery during manipulations. The emanation escaping from the thorium or actinium, diffuses through

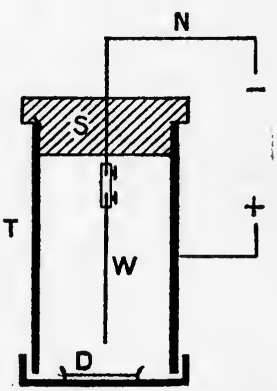

FIG. 39. the cylinder, and as it decays produces active deposit, which is then drawn on to the cathode. After an exposure of a suitable length of time, the wire $W$ is removed.

When an active plate is required, the apparatus shown * Rutherford, Radio-activity, 1912, § 144. 
in figure 40 may be used. 'The active material is contained in the shallow metal dish $A$, which is maintained at a positive potential. The plate $B$, to be made active, serves as the lid of the dish and is maintained negatively charged; a ring of mica $M$ serves to insulate the plate.

To obtain the greatest possible activity, an exposure lasting three hours in the case of actinium and three days in the case of thorium almost suffices. The

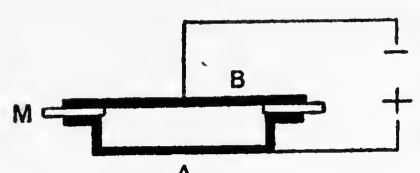

A

FIG. 40. amount of active deposit collected on the wire will depend on the physical state of the preparation used. With dry preparations of thorium and actinium salts, the emanation remains occluded. The escape of the emanation can, however, be insured by keeping the salt slightly damp and using it in a thin layer. In the case of thorium it is best to use the hydroxide, which evolves emanation much more freely than other compounds.

Radium Active Deposit. On account of the long life of the emanation and its tendency to remain occluded in the radium salt, the experimental arrangements just described are unsuitable in the case of radium. When dealing with radium preparations of low activity corresponding to less than $10^{-3} \mathrm{mgr}$. $\mathrm{Ra} \mathrm{Br}_{2}$, the following method may be used.

The radium salt is dissolved in hydrochloric acid. Under these conditions the emanation, as it is formed, escapes from the solution to an appreciable extent. It is advisable to have the solution strongly acid to prevent the gradual precipitation of the radium which may otherwise occur, with the result that the emanation remains occluded. Also the presence of even traces of sulphates must be avoided, since radium sulphate is very insoluble.

The solution is placed in a bottle and kept

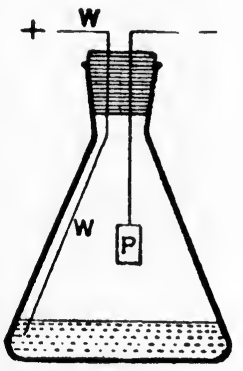

Fig. 41. positively charged by the platinum wire $W$ dipping into it (fig. 41). The plate $P$, to be rendered active, is introduced into the bottle and charged negatively. The manipulation 
should be carried out quickly to prevent the escape of emanation. After an exposure of three hours, almost the greatest possible amount of active deposit will have been collected (\$52).

When working with large quantities of radium, this method of exposure is unsuitable, since it is impossible to prevent occasional losses of emanation. The escape of any appreciable quantity of radium emanation should be avoided, as it diffuses through the room and contaminates any measuring instruments which it reaches. Moreover, by the above method full use is not made of the radium salt, since part of the emanation remains in solution and does not contribute to the activation of the surface. It is generally preferable not to use the radium preparation itself, but to separate the emanation from it and to transfer it into a second vessel in which the exposure takes place. This diminishes the risk of any loss of radium, and makes it possible to use the radium available to the best advantage ( $\$ 75)$.

The gases containing the emanation are collected over mercury in a glass tube $A$, as shown in figure 42 . Fitting tightly into the glass is a tube $F$ of thin iron which is in electrical contact with the mercury. The bent glass tube $T$ has passing through it a wire $W$, which ends in a platinum wire sealed through the glass. The wire $W$, which is insulated from the mercury, is connected to the negative pole of a battery and the mercury to the positive pole. When dealing with large quantities of radium emanation, it is advisable to use a large potential difference in order to collect as much active deposit as possible on the

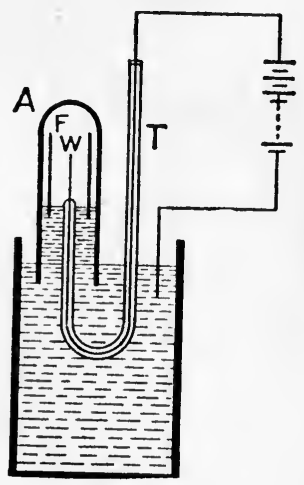

Fig. 42. wire; for with weak fields the active deposit, when formed, moves too slowly through the gas, and may lose its charge by recombination before reaching the cathode. Even an electric intensity of 1000 volts per centimetre may be insufficient to drag out all the active deposit formed when working with quantities of emanation of the order of 100 millicuries. 
In order to be able to detach the wire $W$ from the tube $T$ it is sometimes convenient to divide the tube into two parts and connect them together by a ground joint. Electrical contact can then be made by introducing a small quantity of mercury into the bend of the tube.

When working with large quantities of radium a special room should be set aside for carrying out the manipulations, in order to avoid contaminating measuring instruments with emanation the escape of which can scarcely be avoided. The danger of the experimenter accidentally contaminating instruments by carrying about active matter on his hands and clothes cannot be too strongly emphasised.

\section{$\S 45$. Activity acquired by a Wire in electric Fields of varying Intensity.}

The activity of a wire exposed to an emanation will depend on the sign and magnitude of the electric field to which it is exposed. A comparison of the activities assumed by a wire under different conditions can be made with actinium emanation and the exposure made in the way described (fig. 39). The wire is exposed three times in succession, first uncharged, then negatively and finally positively charged by means of a battery giving about 100 volts. It will be found that the wire is far more active when negatively charged than in the other two cases. When the wire is uncharged, as might be expected, a certain quantity of activity finds its way to the electrode by diffusion, and the exact quantity found there will depend upon the relative areas of the wire and the vessel containing the emanation. Finally, when the wire is positively charged a small quantity of active deposit finds its way to the wire, however great the intensity of the field may be. The measurements are made with an $\alpha$-ray electroscope immediately after removing the wire from the emanation. Some typical results are given below for the case of a wire exposed for 30 minutes to actinium emanation:

Wire negative, 249 divisions per minute.

Wire positive, 4.66 " "

Wire uncharged, 35.2 " " 
The numbers given refer to a case in which the end of the wire was about half a centimetre away from the actinium preparation. The results may be very different if the collecting wire is at a greater distance from the actinium.* The cause of this variation has yet to be explained. The thorium and radium active deposits do not exhibit this peculiarity.

The effect of varying the strength of the electric field should also be studied and a curve drawn showing the amount of active deposit collected by the wire when charged to different potentials. Results obtained with actinium active deposit are shown in figure 43. Similar curves are obtained with thorium and radium active deposits.

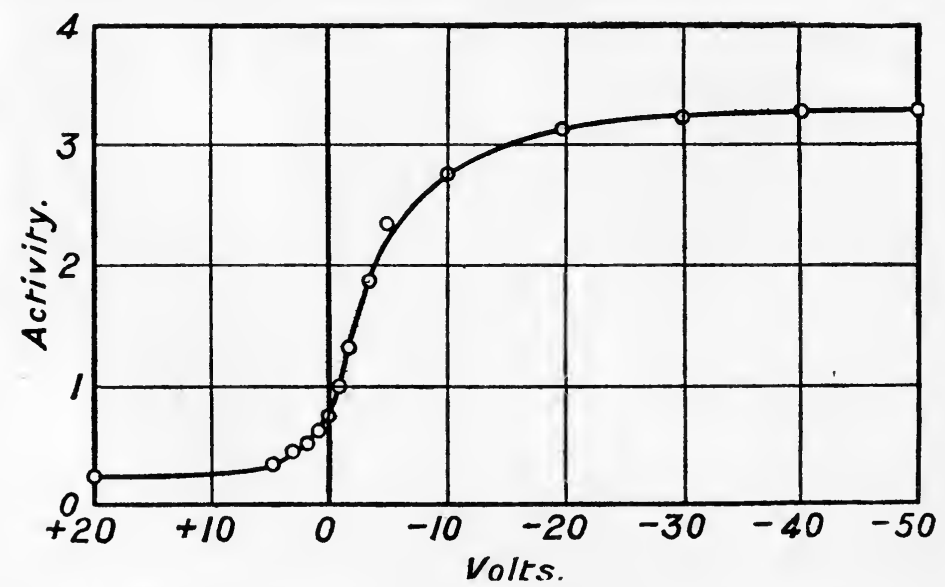

FIG. 43,-Amounts of actinium active deposit concentrated in different electric fields.

\section{$\$ 46$. Radio-active Recoil. $\dagger$}

When a radio-active transformation takes place, involving the expulsion of an $\alpha$ particle, the atom which emits it suffers a considerable impulse; for the $\alpha$ particle is expelled with a very high speed, so that the atom from which it escapes recoils in a direction opposite to that in which the $\alpha$ particle is travelling. The recoiling atoms may even leave the surface upon which the radio-active product is deposited; they con-

* Russ, Phil. Mag. 15.601 and 737, 1908.

† Rutherford, Radio-activity, 1912, §73. 
stitute the next disintegration product in the radio-active series, and are thus separated from their parent substance.

To take an example-suppose that a surface is rendered active by a short exposure to radium emanation, the activity will immediately after removal from the emanation be almost entirely due to radium $A$. In disintegrating, an atom of radium $A$ emits an $\alpha$ particle with a velocity of $1.77 \times 10^{9}$ centimetres per second. From the law of the conservation of momentum, the velocity of the residual radium $B$ atom can be calculated if the atomic weight of radium $B$ is known. Now the atomic weight of radium is 226 , and since radium $B$ is produced from radium as the result of three successive transformations, during each of which an a particle of atomic weight 4 is expelled, the atomic weight of radium $B$ should be 214. If free to move, its velocity of recoil when formed from radium $A$ will therefore be $3.27 \times 10^{7}$ centimetres per second. Thus, if radium $A$ is deposited in a thin film on a plate, it is not surprising that whenever an $\alpha$ particle is shot into the plate the residual atom of radium $B$ formed by the breaking-up of the atom should have sufficient energy to cause it to leave the plate. Thus, half the number of atoms of radium $B$ formed may be projected from the plate and continue to travel away from it till stopped by collision with some obstacle. Now the energy of the recoiling atom compared with that of an $\alpha$ particle is so small that it will penetrate only very slight thicknesses of matter, and air at atmospheric pressure is sufficiently dense to stop the atom of radium $B$ after it has travelled only a tenth of a millimetre. On the other hand, if the experiment is performed in a high vacuum, there will be nothing to impede the flight of the atoms, which may travel large distances before colliding with a solid obstacle, upon which they will then be deposited.

An experimental demonstration of the phenomena described above can be made by exposing a plate in vacuo to a surface coated with radium $A$. ${ }^{*}$ The recoiling atoms of radium $B$ are projected on to the plate. The experiment is, however, difficult on account of the quick decay of radium $A$.

* Russ and Makower, Proc. Roy. Soc. A. 82. 206, 1909. 
The phenomena of recoil may frequently be advantageously employed to separate a radio-active product from its parent product; but the separation can often be effected in a simpler manner than that given above.* We have seen that when a radio-active atom recoils from an $\alpha$ particle, the velocity which it acquires only suffices to carry it a fraction of a millimetre through air at atmospheric pressure before it is stopped by impacts with the molecules of air. Now the recoiling atom has been shown to carry a positive charge, and will therefore be attracted to the cathode in an electric field after being stopped in its flight. This fact can be made use of to concentrate the recoiling atoms on a plate. In figure 44 $M$ represents the plate on which is deposited the active material from which the recoil is to take place. The plate $M$ is kept positively charged, and is separated from the plate $N$ by an insulating

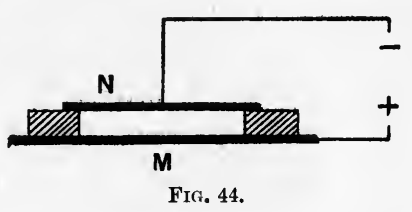
ring. The plate $N$, which is charged negatively, will then collect the atoms which have recoiled from $M$, and will thus become active. The plates $M$ and $N$ should be placed as near together as possible without touching each other, and a potential difference of a few volts maintained between them.

This method of separating radio-active products by recoil can be studied in the case of the active deposit of actinium. The plate $N$ is exposed to the plate $M$, which has previously been rendered active by a long exposure to actinium emanation. After 10 or 15 minutes the plate $N$ is removed and its $\beta$-ray activity tested. The activity of the plate will be found to decay exponentially with a period of 4.7 minutes, showing that pure actinium $D$, which emits only penetrating $\beta$ and $\gamma$ rays, has been deposited on it. A similar experiment can be made with thorium active deposit, whereby thorium $D$ is isolated by the recoil. Thorium $D$ emits $\beta$ and $\gamma$ rays, and decays with a period of $3 \cdot 1$ minutes.

Experiments should be made with various fields between the plates $M$ and $N$ (fig. 44). Taking the case of actinium

* Hahn, Phys. Zeitschr. 10. 81, 1909. 
as typical, it will be found that the amount of actinium $D$ collected on the plate $N$ increases as the field is increased, but reaches a limit for a potential difference of a few volts. In this case practically all the actinium $D$ which recoils from $M$ into the gas is carried by the field to the plate $N$.

Another separation which can be carried out by the method of recoil is that of radium $B$ from radium $A$. A wire or plate is exposed to radium emanation for a few minutes in an electric field, so that a deposit of radium $A$ is formed on it. A second plate is then exposed to the active surface for a few seconds, and quickly transferred to an $\alpha$-ray electroscope. Radium $B$ will be concentrated on the plate, and if the exposure is short, no appreciable amount of radium $C$ will have had time to be formed before making measurements. Since radium $B$ emits no $\alpha$ rays, the activity of the plate will at first be very small. But as radium $C$ is formed, the activity will gradually grow, reaching a maximum after about 32 minutes, and will then fall off again with time. The exact variation of activity with time can be calculated from equation 44 (\$ 51).

\section{$\$ 47$. The Efficiency of Recoil.}

We have seen that when a layer of radio-active material, emitting $\alpha$ rays, is deposited on a plate, the residues of the atoms after the expulsion of the $\alpha$ particles will be projected in directions opposite to those in which the $\alpha$ particles move. Now half the $\alpha$ particles will be shot away from the plate and half into the plate. It thus follows that if every $\alpha$ particle were effective in producing a recoil, half the atoms taking part in the transformation should escape from the plate. But if some of the atoms projected from the plate are in some way stopped before getting away from the surface, the number of atoms escaping from the surface will be less than this theoretical amount. The ratio of the number of atoms escaping to the number which would escape if every recoil were effective, is known as the efficiency of the recoil. It is to be understood that those atoms which are shot towards the plate are disregarded in calculating the efficiency. 
To find the efficiency in the case of the recoil from actinium $C$, a plate is made active by exposure to actinium emanation, and a recoil experiment performed as described above ( $\$ 46)$, making an exposure of about 15 minutes. The $\beta$-ray activities of the two plates are then measured and the readings corrected for the changes of activity in the time which has elapsed since terminating the recoil. Since in the active deposit actinium $D$ alone emits penetrating $\beta$ rays, the $\beta$-ray activity gives a measure of the number of atoms of actinium $D$ present. The ratio of the amount of actinium $D$ on plate $N$ (fig. 44) to half the amount on the plates $M$ and $N$ together gives the efficiency of recoil. It is of importance that the plates $M$ and $N$ should be of the same material, so that the scattered $\beta$ radiation may be the same in all measurements. It will be found that the efficiency of recoil is always less than unity, the exact value depending on the conditions of experiment. The care with which the surface $M$ is cleaned and polished seems to have a great influence on the amount which recoils from it. The efficiency of the recoil of thorium $D$ cannot be calculated as simply as in the case of actinium $D$, since thorium $C$ as well as thorium $D$ emits $\beta$ rays, for which allowance must be made.*

* Marsden and Darwin, Proc. Roy. Soc. A. 87. 17, 1912. 


\section{CHAPTER VII.}

\section{RADIO-ACTIVE TRANSFORMATIONS.}

\section{\$48. Exponential Decay of Radio-active Substances. *}

The theory of the nature of radio-activity first put forward by Rutherford ' and Soddy is too well known to require more than a brief statement. According to this view the emission of radiation by certain substances is the manifestation of atomic disintegration, so that the rate of emission of rays gives a means of estimating the speed at which the disintegration is proceeding. Thus, in radio-active substances, the atoms are constantly breaking up, each atom giving rise to a new atom, while this change is made evident by the emission of $\alpha$ or $\beta$ particles. The new atoms formed possess chemical and physical properties entirely different from those of the parent atoms. In the case of a transformation taking place with the emission of $\alpha$ rays, the new substance has an atomic weight smaller by four units than that of the atom from which it arose; for the a particle has been identified with the helium atom, the atomic weight of which is 4 . On the other hand, if the transformation takes place with the emission of a $\beta$ particle, there seems to be no appreciable change in atomic weight. Nevertheless, the new atom and that from which it arose are entirely distinct in chemical and physical properties.

The general theory of radio-active transformations is based upon the assumption that each substance is disintegrating at a rate peculiar to itself and that the number of atoms breaking up per second, which is measured by the activity of the substance, is at any time proportional to the number of atoms present. Thus if $P_{0}$ represents the number of

* Rutherford, Radio-activity, 1912, Chapters VIII. and XI. 
atoms of any given product present at any time taken as zero, the number of atoms $P$ present after $t$ seconds is given by the equation

$$
P=P_{0} e^{-\lambda t},
$$

where $\lambda$ is a constant which measures the rate of disintegration of the matter. This is at once evident from the following consideration. By differentiating equation (32), we obtain the equation,

$$
\frac{d P}{d t}=-P_{0} \lambda e^{-\lambda t}
$$

and therefore

$$
\frac{d P}{d t}=-\lambda P
$$

Thus, $\lambda$ represents the fraction of the total number of atoms present which undergoes transformation at any moment. It therefore defines the rate of decay, and is known as the transformation constant. Its reciprocal, $\frac{1}{\lambda}$, can be shown to measure the mean life of the atoms. The rate of transformation is sometimes expressed in terms of the mean life, but it is preferable to define the rate of decay of a substance by its transformation constant or by the time $T$ taken for the number of atoms present to decrease to half. The time $T$ is connected with $\lambda$ by the relation

$$
T=\frac{1}{\lambda} \log _{e} 2=0.693 \frac{1}{\lambda} \text {. }
$$

The quantity $T$ is called the half-value period of the substance. From the properties of an exponential curve it is obvious that $T$ remains unaltered as the radio-active substance decays.

It is only in certain cases that a radio-active substance can be isolated from other radio-active bodies and its properties studied by itself; but where this can be done, the activity of the substance is found to decay according to an exponential law, as given by equation (32). Polonium and uranium $X$ afford examples of such cases; but owing to their slow rate of transformation, these substances are inconvenient for testing the above law. On the other hand, actinium $D$ and thorium $D$, which can be isolated by recoil, decay rather R. -A. 
quickly. There are, however, many cases in which complex bodies decay as if they were simple substances. This is the case with the active deposit of actinium collected on a plate by exposure to the emanation for at least two hours. Except for the first few minutes after removal of the plate from the emanation, the $\alpha$-ray activity decays according to an exponential law. The reason of the deviation from the exponential law in the first few minutes is

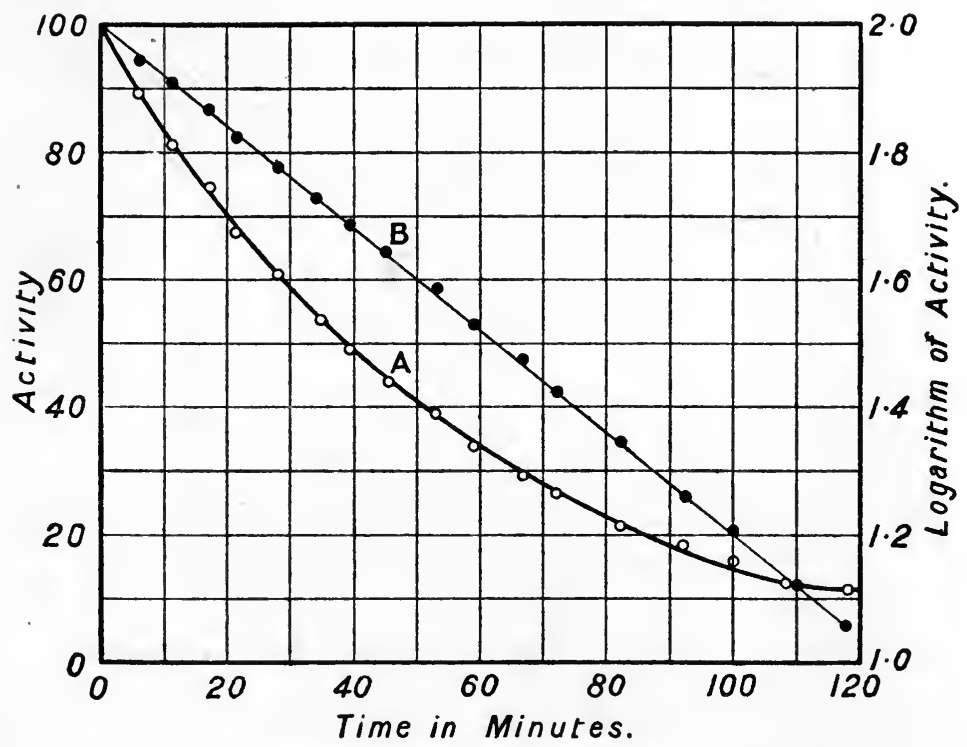

Fig. 45.-Decay of $\alpha$-ray activity of actinium active deposit (long exposure).

given in $\$ 50$. An experiment is carried out by determining the activity with an $a$-ray electroscope at frequent intervals for an hour or two. The activity at different times is represented graphically in figure 45 . It will be seen that the activity decays according to an exponential law falling to half value in every 36.3 minutes (curve $A$ ). The accuracy with which the decay obeys that law can be better tested by plotting the logarithms of the activity at different times against the time, as shown in curve $B$. A straight line will be obtained if the decay is exponential; 
for if logarithms of each side of equation 32 are taken, we obtain the relation,

$$
\log _{e} \frac{P}{P_{0}}=-\lambda t
$$

The value of $\lambda$ can at once be obtained from the slope of this line.

$\$$ 49. Decay of Thorium and Actinium Active Deposits (short exposure).

In the case discussed in $\$ 48$ it was possible to study the transformation of one kind of matter into another by itself; but the process is generally more complicated. The original substance produces a second one, which may itself be radio-active, and will therefore disintegrate in turn, giving rise to a third product. The third substance, if radio-active, will give rise to a fourth product, and so a series of radioactive bodies may be formed as the result of the successive changes. This is, in fact, found to be the case, and three separate series of radio-active substances are known as the result of the disintegration of uranium, actinium and thorium.

The theory of successive radio-active changes can be studied for the cases of the, active deposits of radium, thorium and actinium. The emanations give rise respectively to analogous series of successive products tabulated below (p. 85). Only the first three members of the active deposits need be taken into account when making $\alpha$-ray measurements. As seen from the table, the first and the third members ( $A$ and $C$ ) of the active deposit in each series give out $\alpha$ rays, while the second members emit soft $\beta$ rays, the ionising effect of which is small compared with that of the $\alpha$ rays.

The decay of the active deposit from radium is in practice more complicated than that of actinium or thorium, since the period of radium $A$ is much longer than those of thorium $A$ or actinium $A$; for whereas the period of radium $A$ is 3 minutes, the periods of the latter bodies are 0.14 and 0.002 second respectively. Thus, if a plate which has been exposed to thorium or actinium emanation is removed and examined, unless special methods are employed to work 
with great speed, all the thorium $A$ and actinium $A$ will have disappeared before measurements can be made $(\$ 56)$. Therefore, when measuring with $\alpha$ rays, the deposits behave as if they consisted of two substances, the first of whichthorium $B$ and actinium $B$-emit soft $\beta$ rays, and the second-thorium $C$ and actinium $C$-emit $\alpha$ rays. It is therefore well to consider these two simpler cases before studying the behaviour of the active deposit from radium.

Suppose that a plate is exposed in an electric field for a few minutes to the emanation from a preparation of mesothorium in the usual way and then removed. The thorium

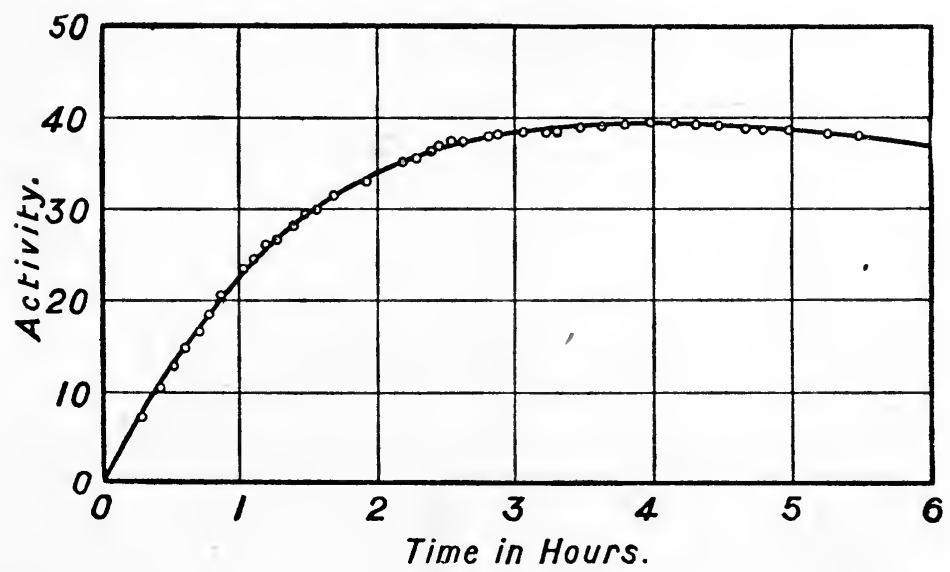

FIG. 46.-Variation of $\alpha$-ray activity of thorium active deposit (short exposure).

$A$ deposited on the plate will at once be converted into thorium $B$. Let the active plate be quickly transferred to an $\alpha$-ray electroscope and measurements of its activity be begun at once. Since thorium $B$ emits no $\alpha$ rays, the activity of the plate will at first be very small; but the activity will rise for the first four hours and then fall off with time, at first slowly and then more quickly, until finally the activity will decay with the period of thorium $B$ (fig. 46). Neglecting the influence of thorium $A$, the rate at which the activity varies with time can be calculated as follows.

Suppose that, having started with a quantity of pure thorium $B$, the thorium. $C$ formed from it breaks up in turn 


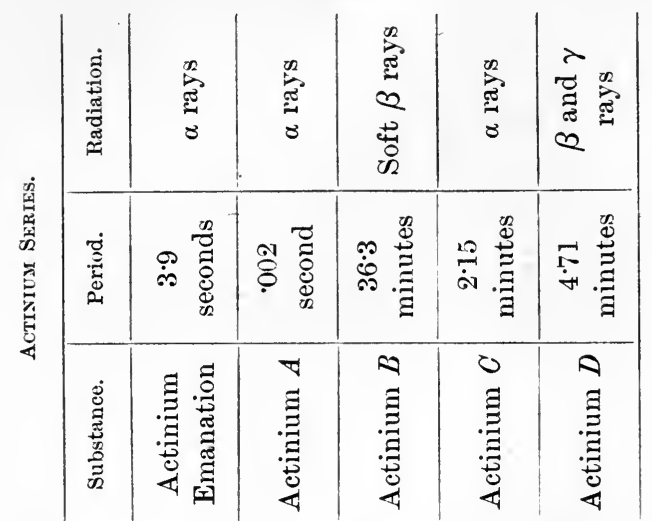

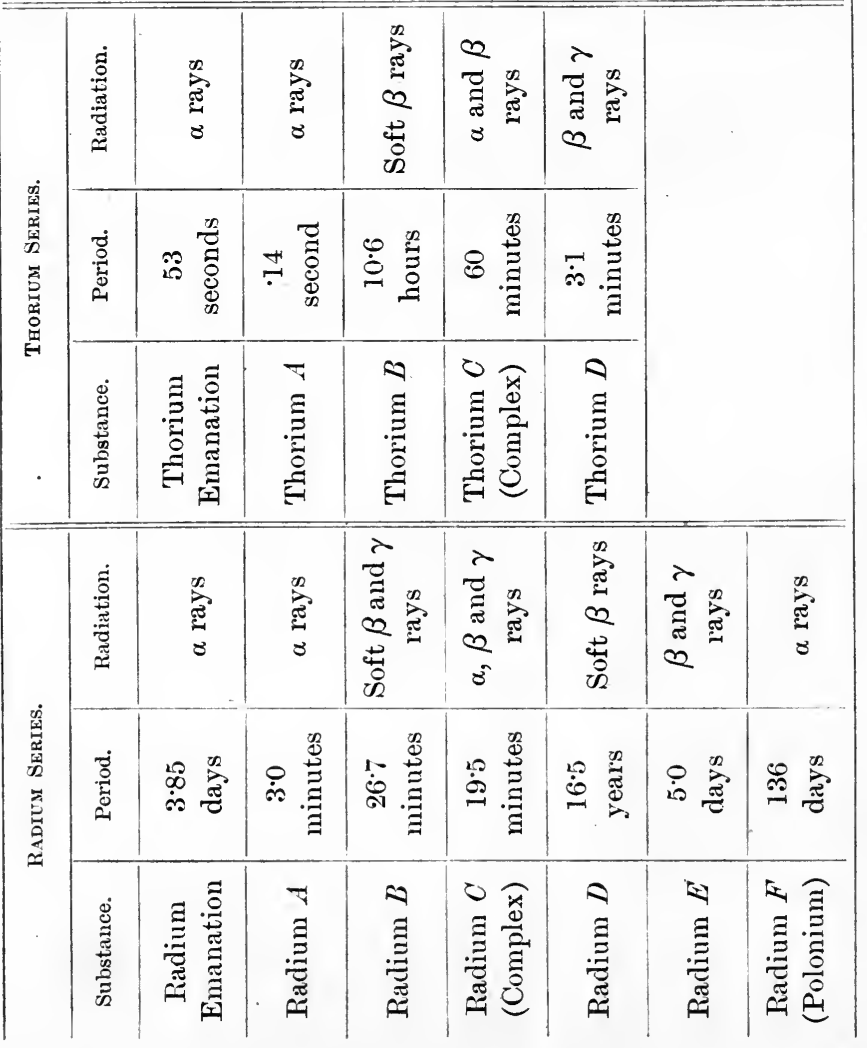


into thorium $D$; thus, if $P$ and $Q$ denote the numbers of atoms of thorium $B$ and of thorium $C$ respectively present at any moment, then the rate of increase of thorium $C$ will be given by the difference in the number of atoms of $C$ formed from $B$ and the number of atoms of $C$ changing into $D$, so that if $\lambda_{1}$ and $\lambda_{2}$ are the transformation constants of $B$ and $C$ respectively,

$$
\frac{d Q}{d t}=\lambda_{1} P-\lambda_{2} Q
$$

where the value of $P$ is determined by the equation,

$$
P=P_{0} e^{-\lambda_{1} t}
$$

Having regard to the fact that $Q=0$ when $t=0$, the solution of this equation can be shown to be

$$
Y=\frac{P_{0} \lambda_{1}}{\left.\lambda_{2}-\lambda_{1}\right]}\left[e^{-\lambda_{1} t}-e^{-\lambda_{2} t}\right] .
$$

By inserting the disintegration constants of thorium $B$ and thorium $C$ for $\lambda_{1}$ and $\lambda_{2}$ respectively, the theoretical curve can be plotted and compared with that obtained by experiment. The time at which the maximum occurs can easily be calculated; for, by differentiating equation (39) and equating to nought, we get the relation,

$$
t_{m}=\frac{1}{\lambda_{1}-\lambda_{2}} \log _{e} \frac{\lambda_{1}}{\lambda_{2}}
$$

The experimental results may be used to calculate the two transformation constants in equation (39). To do this two relations are required, the first of which is obtained by determining the rate of decay when the time $t$ has become large. For one of the exponential terms in equation (39) then becomes small compared with the other one, and the decay follows a simple exponential law, governed by the period of the product of longer life. In the case of thorium active deposit, the second term becomes negligible compared with the first after about four hours. In this way $\lambda_{1}$ is found.

To obtain $\lambda_{2}$ a second relation is necessary. For this purpose the time at which the maximum occurs is determined and its value inserted in equation (40). It is, however, difficult to fix with accuracy the moment of the occurrence of the 
maximum, and the following method of finding $\lambda_{2}$ is therefore more often adopted.

It will be seen from equation (39) that the activity curve is made up of two exponentials. Hence, by extrapolation of the end portion $B C$ of the experimental curve, an exponential is constructed back to zero time, as shown by the curve $D B$ in figure 47. If the experimental curve $A B C$ is now subtracted from the curve $D B C$ so obtained, another exponential $E E$ will result, the time constant of which is $\lambda_{2}$.

The variable terms within brackets in equation (39) is are symmetrical with respect to $\lambda_{1}$ and $\lambda_{2}$. It therefore follows

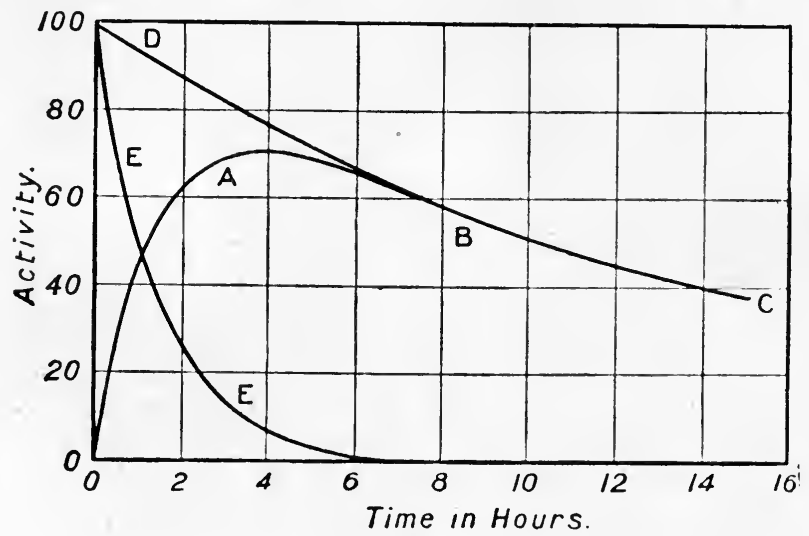

FIG. 47.-Thorium active deposit : analysis of activity curve.

that no information can be obtained as to whether thorium $B$ or thorium $C$ has the longer period. To decide this point it is necessary to separate the products from each other and to examine their rate of decay separately (\$74). Although the periods of the two elements cannot be assigned to the respective products, it is obvious that the $\alpha$ rays must be emitted by thorium $C$, otherwise the curves would not show the rise actually observed during the first few hours.

The theory of a short exposure to the actinium emanation is the same as that for thorium given above. In this case, however, the maximum occurs after 9 minutes, for which reason the exposure must not last longer than a fraction of a 
minute. A curve showing the variation of activity with time is given in figure 48. The rapid rise of activity in the first few minutes necessitates accurate measurements made in quick succession.

Similar results are obtained with the active deposits of actinium and thorium when measuring with penetrating $\beta$ rays, which arise from the products actinium $D$ and thorium $C$ and thorium $D$. It at first sight appears that the equations

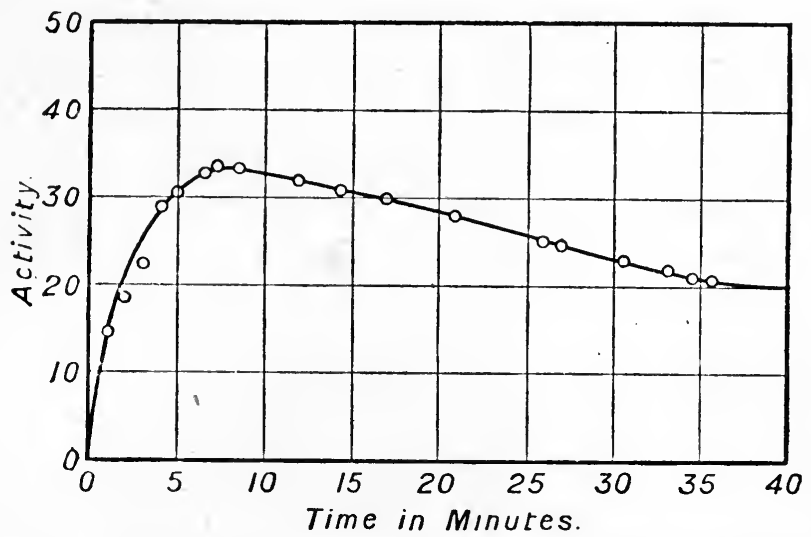

FIG. 48. - Variation of $a$-ray activity of actinium active deposit (short exposure).

do not apply, since starting with $B$ there are three successive products involved in the transformations. However, the periods of actinium $D$ and thorium $D$ are short, and therefore the deposits behave almost as if the $\beta$ radiation came from actinium $C$ and thorium $C$.

\section{$\$ 50$. Decay of Thorium and Actinium Active Deposits (long exposure).}

In $\S 49$ the case has been considered in which the radioactive matter is initially all of one kind. There is, however, another case of practical importance. Taking again the case of thorium, suppose that a surface has been exposed to the emanation for four or five days and is then tested. Whereas for a short exposure thorium $B$ was alone present at the beginning of the measurements, we now have also the products formed from it. For during the exposure the thorium $A$ will 
have had time to be transformed into thorium $B$ and its successive products. The number of atoms of each product present will have continued to rise until its rate of decay is balanced by its rate of production from the substance preceding it. When this condition has been reached, the same number of atoms of each product must be decaying per second, and the products are said to be in radio-active equilibrium.

Let $n_{0}$ be the number of particles of thorium $A$ deposited per second from the source. If equilibrium has been established, this quantity must be equal to the number of atoms of $B$ changing into $C$ and of $C$ changing into $D$, so that

$$
n_{0}=\lambda_{1} P_{0}=\lambda_{2} Q_{0}=\lambda_{3} R_{0}
$$

where $P_{0}, Q_{0}, R_{0}$ are the quantities of $B, C$ and $D$ present when equilibrium has been reached. Thus the numbers of the atoms of the different products present are inversely proportional to their respective decay constants. When the surface exposed is removed from the emanation, equilibrium is disturbed. The numbers of atoms of thorium $B$ and thorium $C$ present at any subsequent moment can then be calculated as follows.

Combining the initial conditions given in equation with equations (37) and (38), we obtain the equation,

$$
Q=\frac{\lambda_{1} P_{0}}{\lambda_{2}-\lambda_{1}}\left[e^{-\lambda_{1} t}-\frac{\lambda_{1}}{\lambda_{2}} e^{-\lambda_{2} t}\right]
$$

which gives the number of atoms of thorium $C$ present at any moment after the termination of the exposure. It follows from equation (42) that the activity at first falls off rather slowly, but with increasing time approximates more and more to an exponential falling off with the period of thorium $B$. From the curve obtained, the values of $\lambda_{1}$ and $\lambda_{2}$ can be deduced in a manner similar to that described for the case of a short exposure ( $\$ 49)$.

The same consideration applies to the case of actinium, but the deviation from the simple exponential immediately after terminating the exposure is small and confined to the first few minutes. 


\section{$\$ 51$. Decay of the Active Deposit of Radium.}

The case of the active deposit of radium is more complicated than that of actinium or that of thorium, since the period of radium $A$ is comparable with the periods of the products which follow it. Moreover, since radium $A$ and radium $C$ both emit $\alpha$ rays, whereas radium $B$ and radium $C$ emit $\beta$ rays, the activity curves will be entirely different when measuring with $\alpha$ rays and when measuring with $\beta$ rays.

By a method of reasoning similar to that given in $\S 49$ it can be shown that if $P, Q$ and $R$ represent the numbers of atoms of radium $A$, radium $B$ and radium $C$ present at any time $t$, the amount of each product will vary according to the differential equations,

$$
\left.\begin{array}{l}
\frac{d P}{d t}=-\lambda_{1} P \\
\frac{d Q}{d t}=\lambda_{1} P-\lambda_{2} Q \\
\frac{d R}{d t}=\lambda_{2} Q-\lambda_{3} R
\end{array}\right\} \ldots \ldots \ldots \ldots \ldots \ldots \ldots . .
$$

Bateman * has given a general solution of these equations, by means of which can be calculated the numbers of atoms of radium $A$, radium $B$ and radium $C$ present at any time after an exposure of any duration. There are two cases of special importance which will be considered below : firstly, that of a short exposure of a surface to radium emanation when only radium $A$ will have had time to be deposited; secondly, that of an exposure long enough to allow all the products to come into radio-active equilibrium.

In the first case $Q_{0}=R_{0}=0$, and the equations become :

$$
\left.\begin{array}{rl}
P & =P_{0} e^{-\lambda t}, \\
Q=\frac{\lambda_{1} P_{0}}{\lambda_{2}-\lambda_{1}}\left[e^{-\lambda_{1} t}-e^{-\lambda_{2} t}\right], & \\
R=\lambda_{1} \lambda_{2} P_{0}\left[\frac{1}{\left(\lambda_{2}-\lambda_{1}\right)\left(\lambda_{3}-\lambda_{1}\right)} e^{-\lambda_{1} t}\right. & +\frac{1}{\left(\lambda_{1}-\lambda_{2}\right)\left(\lambda_{3}-\lambda_{2}\right)} e^{-\lambda_{2} t} \\
& \left.+\frac{1}{\left(\lambda_{1}-\lambda_{3}\right)\left(\lambda_{2}-\lambda_{3}\right)} e^{-\lambda_{3} t}\right]
\end{array}\right\}
$$

* Bateman, Proc. Camb. Phil. Soc. 15. 423, 1910. 
In the second case of a long exposure, we have initially,

whence

$$
\lambda_{1} P_{0}=\lambda_{2} Q_{0}=\lambda_{3} R_{0}
$$

$$
\left.\begin{array}{rl}
P & =P_{0} e^{-\lambda t}, \\
Q & =Q_{0}\left[\frac{\lambda_{2}}{\lambda_{2}-\lambda_{1}} e^{-\lambda_{1} t}+\frac{\lambda_{1}}{\lambda_{1}-\lambda_{2}} e^{-\lambda_{2} t}\right], \\
R=R_{0}\left[\frac{\lambda_{2} \lambda_{3}}{\left(\lambda_{2}-\lambda_{1}\right)\left(\lambda_{3}-\lambda_{1}\right)} e^{-\lambda_{1} t}+\frac{\lambda_{1} \lambda_{3}}{\left(\lambda_{1}-\lambda_{2}\right)\left(\lambda_{3}-\lambda_{2}\right)} e^{-\lambda_{2} t}\right. \\
& \left.+\frac{\lambda_{1} \lambda_{2}}{\left(\lambda_{1}-\lambda_{3}\right)\left(\lambda_{2}-\lambda_{3}\right)} e^{-\lambda_{3} t}\right] .
\end{array}\right\}
$$

The numbers of atoms of radium $A, \operatorname{radium} B$ and radium $C$ present at any time after a short or a long exposure to the emanation can thus be found from equations (44) and (46). Since radium $A$ and radium $C$ emit $\alpha$ rays, the $\alpha$-ray activity $M$, being proportional to the number of atoms disintegrating per second, is given by

$$
M=\lambda_{1} P+k \lambda_{3} R,
$$

where $k$ is the ratio of the ionisation produced by an $\alpha$ particle from radium $C$ to that produced by an $\alpha$ particle from radium $A$ under the conditions of the experiment.

Consider first the case in which a plate is exposed to radium emanation for a few seconds. The active matter deposited will consist of $\operatorname{radium} A$ only, and thus be all of one kind. If the plate is quickly transferred to an $\alpha$-ray electroscope, the activity will initially be entirely due to the rays from radium $A$, and will therefore begin to fall off with a half-value period of 3 minutes. ${ }^{*}$ The fall will continue until radium $C$ is being formed from radium $B$ in sufficient quantity to counteract the decay of the radium $A$, when the activity will slightly rise for some time, after which the activity again falls off with time. Ultimately, after several hours the activity decreases with the period of radium $B$, falling to half value in $26 \cdot 7$ minutes.

* In transferring the wire small quantities of emanation often adhere to it. The emanation ean be driven off by raising the wire in vacuo to a temperature of about $400^{\circ} \mathrm{C}$. for a few seconds. The emanation can also be partially removed by washing the wire with alcohol. 
If the exposure of the surface to the emanation is long, extending over several hours, the initial drop of activity is less marked, as a considerable quantity of radium $C$ has been accumulated before beginning measurements. The theoretical curves calculated from equation (47) for short and long exposures, as measured by $\alpha$ rays, are given in figure 49 . In the equation the value of $k$ is taken unity. Curve $I$ refers to the case of a long exposure and curve $I I$ to that of a very short exposure.

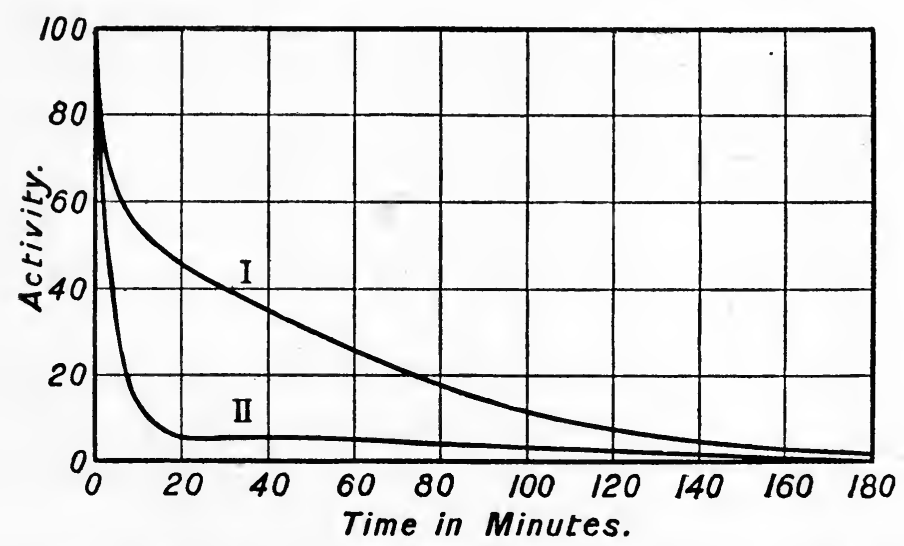

Fic. 49.-Variation of $\alpha$-ray activity of radium active deposit (long and short exposures).

When measuring with $\beta$ rays, the total activity, $N$, at any time will be given by

$$
N=\lambda_{2} Q+l \lambda_{3} R
$$

where $l$ is the ratio of the ionisation produced by a $\beta$ particle from radium $C$ to that produced by a $\beta$ particle from radium $B$. The shape of the curves will depend upon the thickness of the screen through which the rays have to pass before entering the electroscope, for radium $C$ and radium $B$ emit $\beta$ rays of different penetrating powers. Now the bulk of the radiation from radium $B$ is much softer than that from radium $C$, so that if the rays have to penetrate more than one millimetre of aluminium, the ionisation produced will for the most part be due to radium $C$. The curves obtained can be calculated from equation (48) by inserting the appropriate value of $l$ 
for the particular thickness of aluminium used. The curves $I$ and $I I$ given in figure 50 represent the cases of long and short exposures, in which the radiation measured is due to radium $C$ only. This case can not be exactly realised in practice on account of a small quantity of hard radiation from radium $B$. But this radiation amounts to only about 5 per cent. of the total radiation, and therefore only very slightly influences the activity curves.*

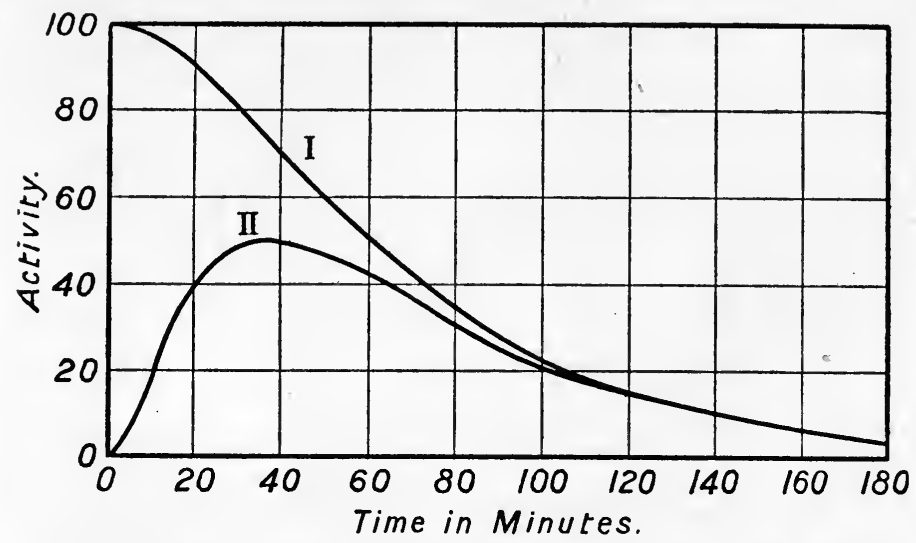

FIc. 50.-Variation of activity of radium active deposit measured by the rays from radium $c$ (long and short exposures).

Equation (48) also applies to the case of measurements of the $\gamma$ radiation, which arises from radium $B$ and radium $C$. The $\gamma$ radiation from radium $B$ is much softer than that from radium $C$, and is practically cut out by $2.5 \mathrm{cms}$. of lead. When measured through this thickness of lead, the variation of activity is represented by the curves given in figure 50 .

\section{$\$ 52$. Decay and Recovery of Radio-active Bodies.}

The continuous growth and decay proceeding in radio-active matter can be studied by separating an active substance from its radio-active parent, and simultaneously measuring the variation of activity of each. Take, for example, the case of the transformation of uranium into uranium $X$, in which uranium gives out only $\alpha$ rays and uranium $X$ only $\beta$ rays. If all measurements are made with $\beta$ rays, then * Fajans and Makower, Phil. Mag. 23. 292, 1912. 
the ionisation produced will depend on the quantity of uranium $X$ present and not on the quantity of uranium; the uranium will be effective only in so far as it produces uranium $X$ and so indirectly influences the radiation to be measured.

Suppose that a sample of uranium has been left for some months so as to come into equilibrium with the uranium $X$ produced by it which has a period of 25 days. If then the

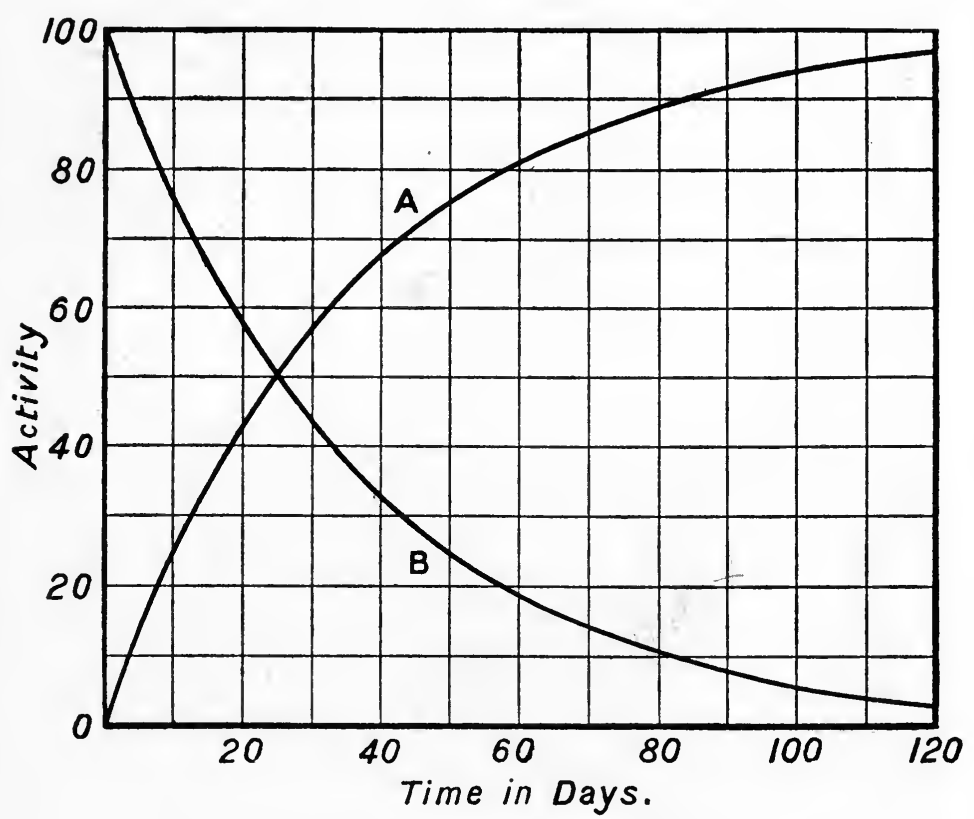

F1G. 51.-Decay and recovery of uranium $X$.

uranium $X$ contained in the uranium is completely separated, the $\beta$-ray activity of the uranium will at first be zero, but will gradually rise as uranium $X$ grows again. After some months its activity will have regained its original value (curve $A$, fig. 51). In the mean time the uranium $X$ which has been separated will fall off exponentially with time, and ultimately become negligibly small (curve $B$ ). The sum of the $\beta$-ray activities of the uranium and the uranium $X$ will always be constant, showing that uranium $X$ is being reformed from 
the uranium at exactly the same rate as the uranium $X$ is decaying. Thus, if $\lambda$ is the constant of disintegration of uranium $X$, the quantity of this substance left in the portion separated from the uranium will at any time $t$, after separation, be given by the equation,

$$
Q=Q_{0} e^{-\lambda t}
$$

where $Q_{0}$ is the quantity of uranium $X$ originally separated from the uranium. It follows at once from the fact that the sum of the quantity of uranium $X$ in the portion separated from the uranium, and the quantity associated with it is constant, that the quantity of uranium $X$ associated with the uranium after a time $t$ is given by the equation,

$$
Q=Q_{0}\left(1-e^{-\lambda t}\right) \text {. }
$$

The experiment is carried out by separating the uranium $X$ from about 10 grams of uranium oxide by the method described in $\S 66$. But the scales of the two curves obtained will be different, since the absorption of the $\beta$ rays by the thick layer of uranium oxide is large compared with the absorption by the film of uranium $X$. This experiment, though instructive, involves taking readings over several weeks. There is, however, no other substance which lends itself so well to illustrate the point.

The relation which exists between the rate of decay of activity of uranium $X$ and the rate of recovery of the uranium from which it has been separated holds quite generally. Thus, if the active deposit has been completely removed on a wire from a tube containing radium emanation, the numbers of atoms of $\operatorname{radium} A$, radium $B$ and radium $C$ on the wire will decrease according to equation 46 , and the wire will ultimately become inactive. While this is taking place, the active deposit will be regenerated in the tube, and the number of atoms of each product present in the tube at any moment will be complementary to the number of atoms of the same product on the wire. This statement is equivalent to saying that the number of atoms of each product inside and outside the tube is constant. Hence if $f(t)$ represents the variation of activity with time of the decaying product, the rate at which the 
product is regenerated in the tube is given by $1-f(t)$, if the slow decay of the emanation is neglected. When $f(t)$ has become small compared with unity, the function $1-f(t)$ will almost have reached a constant limiting value. The time at which this occurs for each product determines the duration of exposure necessary to obtain the maximum possible amount of that product. Thus the amount of radium $C$ on a wire after a long exposure to radium emanation decays to about one per cent. in two hours, while in the same time 99 per cent. of the equilibrium amount will have accumulated.

\section{$\$$ 53. Rate of Decay of Actinium $C$.}

Attention has been drawn in $\$ 49$ to the fact that, although the values of the decay constants of successive radio-active products can be deduced from the shape of the decay curves, the constants cannot be assigned to their respective products. To do so it is necessary to separate the products from each other.

It has already been seen that the $\alpha$-ray decay curves of actinium active deposit are controlled by two products whose periods are 36.3 and $2 \cdot 1$ minutes, and also that the second product, actinium $C$, emits $\alpha$ rays, whereas actinium $B$ does not. The products can be separated by immersing a nickel plate in a solution of the active deposit $(\$ 72)$. The nickel plate, after removal, is found to emit $\alpha$ rays at a rate decreasing with a period of $2 \cdot 1$ minutes. Consequently the second product, actinium $C$, decays with this period, while actinium $B$ decays to half value in 36.3 minutes.

Actinium $C$ may also be partially separated by one of the following general methods. In the first method advantage is taken of the fact that actinium $C$ is much less volatile than actinium $B$. Thus, if a platinum plate which has been exposed to actinium emanation is heated for a few seconds to about $800^{\circ} \mathrm{C}$., actinium $C$ will be left on the plate in excess, and the $\alpha$-ray activity will decay rapidly at first, showing again that the product which emits the $a$ rays has the shorter period. .In the second method actinium $B$ and actinium $D$ are dissolved off the plate, leaving the actinium $C$ alone. The experiment is carried out by immersing a platinum plate coated with the 
actinium active deposit in dilute hydrochloric or sulphuric acid. The solution should be made up to about $\frac{1}{10}$ normal and the plate immersed for a few seconds. On transferring the plate to an $\alpha$-ray electroscope, its activity will again be found at first to decay rapidly, showing that actinium $B$ has been partially dissolved by the acid.*

\section{$\$ 54$. Comparison of the Rates of Decay of the Emanations.}

The emanations from radium, thorium and actinium have very different rates of decay. This can be illustrated by blowing them into an emanation electroscope (fig. 17) and observing the rate of disappearance of the activity in each case.

Thorium and actinium emanations are most easily introduced by bubbling air through a solution of the active material into the electroscope. After the current has been allowed to pass for about a minute, the electroscope is closed and the variation of activity at once measured. With thorium emanation it will be found that the activity falls to half value in 53 seconds; with actinium emanation the whole activity of the emanation disappears in a few seconds, leaving a small residual activity due to the active deposit. The rate of decay of actinium emanation is too rapid to be measured in this way, but the determination can be made by a method given in $\S 55$.

A solution containing a few grams of thorium nitrate produces sufficient emanation, provided that the gas does not take too long in travelling from the solution to the electroscope. This condition is obviously still more important in the case of actinium.

The emanation of radium is introduced in a similar manner by bubbling air through a solution of radium into the electroscope. The activity first rises for about four hours on account of the formation of the active deposit, and subsequently falls off with a period of 3.85 days, which is characteristic of that emanation. A minute quantity of radium is sufficient for the purpose (\$62). With radium the electroscope must first be partially exhausted and the air carrying the emanation allowed to flow into the electroscope until atmospheric pressure is

$$
\text { * Schrader, Phil. Mag. 24. 125, } 1912 .
$$

R.-A. 
established. It is important in this case to prevent the escape of emanation, since the solution contains only a definite amount of emanation, which, when withdrawn, takes a long time to be regenerated. With thorium and actinium, on the other hand, the emanations are replaced very rapidly.

\section{$\$ 55$. Rate of Decay of Actinium Emanation.}

When dealing with a radio-active substance, the life of which is short, special methods have to be used to determine its rate of decay. Actinium emanation decays to half value in 3.9 seconds, and the following method of finding its rate of decay serves as an example of the manner of dealing with short-lived products.

To determine the rate of decay of actinium emanation the apparatus shown in figure 52 is used. A preparation of actinium is placed in a dish $A$ at one end of a metal tube

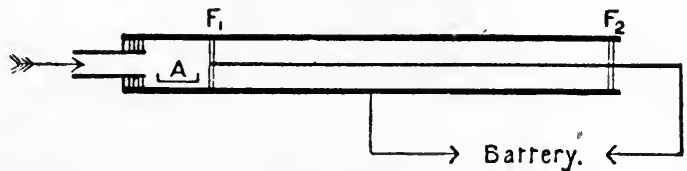

FI\%. 52.

about 40 centimetres long and one centimetre in diameter. A wire is fixed along the axis of the tube and held in position by two small ebonite supports $F_{1} F_{2}$. A field is maintained between the tube and the wire by a battery giving about 100 volts, the wire being connected to the negative pole of the battery and the tube to the positive. A constant current of air is then maintained along the tube and carries the actinium emanation with it. Since the emanation during its passage along the tube is exposed to an electric field, the active deposit produced at different sections along the tube will at once be drawn to the central electrode. Since the emanation decays to a measurable extent in its passage through the tube, the amount of active deposit on the wire will be appreciably different at different points.

The rate of flow is so regulated that the time taken by the emanation to travel along the whole length of the tube is several times greater than 399 seconds, the half-value constant 
of the emanation. After the current has been flowing for about 2 hours, the activity on the wire will have practically reached a maximum, since the half-value period of actinium $B$ is 36.3 minutes ; for it is this product which, having the longest life, controls the rate of growth of the active deposit. After the exposure the wire should be removed and cut up into pieces about one or two centimetres long, and each piece tested in an $\alpha$-ray electroscope. The activities of the pieces then give the distribution of the emanation along the tube during the passage of the air current. It is of course necessary to apply a correction for the decay of the active deposit, since all the pieces into which the wire has been cut cannot be tested simultaneously. The rate of decay of the activity will be exponential, and the activity will fall off with a half-time constant of 36.3 minutes (Appendix II.). Having tested the wire at different points as described above, it is only necessary to know the rate of flow of the emanation along the tube to calculate the rate of decay of the emanation.

A constant current of gas can be maintained by a large gasometer, or more conveniently by the apparatus shown in figure 53 . A large bottle $A$, which is completely filled with water at the beginning of the experiment, communicates through the tap $E$ with another similar bottle $B$. The bottle $B$, which is empty at the beginning of the experiment, has three tubes passing through its stopper-one communicating with the tap $E$ and two others, $F$ and $H$, as shown in the figure. When ready to begin the experiment the tap $C$ is closed and $E$ is opened.

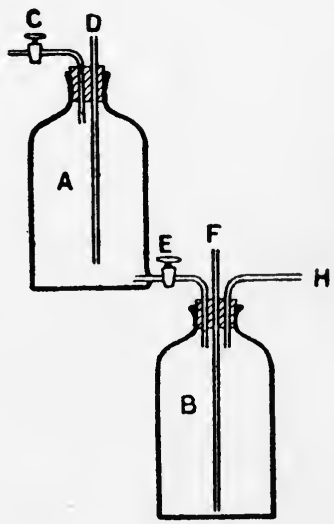

FIG. 53. Water will flow into $B$ and air will be sucked in through $D$ to replace the water. It is to be noticed that since the tube $D$ reaches nearly to the bottom of the bottle $A$, the water will always be flowing out through $E$ at a constant pressure just greater than atmospheric, and will force through 
$H$ a constant stream of air at a rate which is independent of the height of the water in the bottle $A$. Care must of course be taken to make all the joints in the apparatus air-tight. At the end of the experiment the water in $B$ can be transferred again into $A$ by connecting the tube $F$ with $D$ by a rubber tube, opening the $\operatorname{tap} C$, closing $E$ and applying a water-pump to $C$.

\section{$\$ 56$. Rate of Decay of very short-lived Products.}

The methods hitherto adopted for determining the rate of decay of radio-active substances are not applicable when dealing with very rapidly decaying products. The case of thorium $A$ affords an illustration of the way of measuring the half-value period of an $\alpha$-ray product of very short life. The method used consists essentially in concentrating the active material on a rotating wire, the activity of which is measured at different distances from the place at which the active material is collected. From a knowledge of the speed of rotation of the wire, the rate of decay of the product can be deduced.

The apparatus shown in figure 54 can be used for thorium $A$, which falls to half value in $\cdot 14$ second. An endless wire

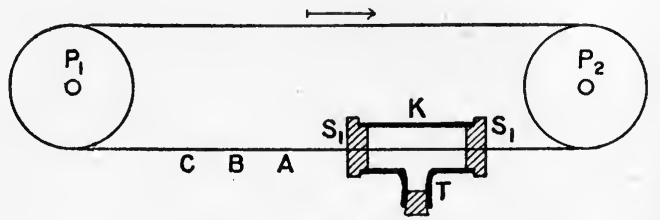

FIG. 54.

passes over the pulleys $P_{1}, P_{2}$ and through a metal cylinder $K$ about $10 \mathrm{cms}$. long and $1 \mathrm{~cm}$. wide. The wire, which is maintained at a negative potential, is guided and insulated by two ebonite stoppers $S_{1}$ and $S_{2}$. The emanation evolved from a strongly active preparation, such as a small quantity of mesothorium contained in the side tube $T$, produces thorium $A$, which, being charged positively, is attracted to the wire as soon as it is formed. When the wire is kept rotating, the thorium $A$ will be carried some distance before losing its 
activity. The activity along the wire can be directly observed by counting the scintillations at different positions $A, B, C$. Hence the decay constant of thorium $A$ can be deduced from the known speed of the wire and from the distances between the points at which the observations were taken. The wire should not move at a speed exceeding a few centimetres per second.

The period of actinium $A$, which is only $\frac{1}{50} \overline{0}$ second, has been determined by a similar method; but the experiment is very difficult.

\section{\$57. Diffusion of Thorium and Actinium Emanations.*}

The rates of diffusion of the emanations have been measured and compared with the rates of diffusion of ordinary gases. The results obtained lead to the conclusion that the emanations are gases of high molecular weight. Diffusion measurements, with the slowly decaying radium emanation, are somewhat complicated to carry out, but the diffusion coefficients of thorium and actinium emanations can be determined by a method first used by Rutherford.

The apparatus employed for the purpose is shown in figure 55. A thin $\operatorname{rod} A$ passes through the insulating plug $D$ along the axis of the brass tube $P$, which is about $15 \mathrm{cms}$. long and $6 \mathrm{cms}$. wide. The thorium preparation is spread uniformly in the dish $C$, which just fits into the tube. The $\operatorname{rod} A$ is connected to the negative pole and the tube to the positive

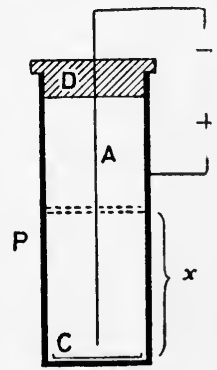

Fig. 55. pole of a battery of storage cells. The emanation diffuses away from the source, and as it decays gives rise to the active deposit, which is immediately dragged along the lines of force to the wire. The distribution of the active deposit along the wire is therefore the same as that of the emanation at different distances from the source.

Consider a section of the cylinder of unit area and of thickness $d x$; let $k$ be the diffusion coefficient and $N$ the number of emanation atoms per unit volume at a distance $x$

\footnotetext{
* Rutherford, Radio-activity, 1912, § 139 et seq.
} 
from the thorium preparation. When a steady state has been reached, the amount of emanation diffusing into the section of thickness $\delta x$ in time $\delta t$ is $k \frac{d N}{d x} \delta t$, while the amount flowing out in the same time is

$$
k \frac{d(N+\delta N)}{d x} \delta t
$$

consequently the quantity of emanation contained within the two planes at distances $x$ and $x+\delta x$ from the thorium is given by the difference between the two above quantities, and is therefore $k \frac{d^{2} N}{d x^{2}} \delta t \delta x$. Now this quantity must decay in situ, and therefore be equal to $-\frac{d N}{d t} \delta t \delta x$. We therefore get the equation,

$$
k \frac{d^{2} N}{d x^{2}}=-\frac{d N}{d t} .
$$

Now if $\lambda$ is the decay constant of the emanation,

$$
-\frac{d N}{d t}=\lambda N
$$

whence

$$
k \frac{d^{2} N}{d x^{2}}=\lambda N
$$

The solution of this equation is of the form,

$$
N=A e^{-\sqrt{\frac{\lambda}{k}} x}+B e^{\sqrt{\frac{\lambda}{k}} x},
$$

and since $N=0$ when $x=\infty$, it follows that $B=0$.

If $N_{0}$ is the number of atoms of emanation per unit volume just above the thorium, then $N=N_{0}$ when $x=0$, so that $A=N_{0}$.

Thus

$$
N=N_{0} e^{-\sqrt{\frac{\lambda}{k}} x} \text {. }
$$

Now the amount of the active deposit at different points along the rod to be tested is proportional to $N$, and therefore falls off exponentially with the distance. Hence, assuming the value of $\lambda$, the coefficient $k$ can be deduced.

In order to avoid disturbances by air currents, the apparatus should be enclosed in a box packed with cotton wool. A few grams of thorium hydroxide or preferably a preparation of mesothorium or radiothorium should be used; the 
exposure should last about 24 hours, which suffices to collect a sufficient quantity of active deposit. In order to determine the distribution of activity, the wire is cut into lengths of about five millimetres each. These are then tested in an $\alpha$-ray electroscope and their activities plotted against their mean distances from the thorium preparation. It will be found that the activity falls to half value for about every two centimetres along the wire. Some typical results are given in the following table:

Table showing the Diffusion of Thorium Emanation.

(Length of sections of wire $=4.9 \mathrm{~mm}$.)

\begin{tabular}{c|c}
\hline $\begin{array}{c}\text { Average Distance } \\
\text { from Source in Millimetres. }\end{array}$ & Activity of Wire. \\
\cline { 2 - 2 } $4 \cdot 9$ & $45 \cdot 8$ \\
$9 \cdot 8$ & $38 \cdot 0$ \\
$14 \cdot 7$ & $32 \cdot 3$ \\
$19 \cdot 6$ & $27 \cdot 8$ \\
$24 \cdot 5$ & $23 \cdot 3$ \\
$29 \cdot 4$ & $20 \cdot 3$ \\
$34 \cdot 3$ & $16 \cdot 7$ \\
$39 \cdot 2$ & $14 \cdot 0$ \\
$44 \cdot 1$ & $12 \cdot 7$ \\
$49 \cdot 0$ & $10 \cdot 9$ \\
$53 \cdot 9$ & $9 \cdot 3$ \\
$58 \cdot 8$ & $7 \cdot 6$ \\
\hline
\end{tabular}

'The diffusion coefficient calculated from these numbers is $0 \cdot 115$.

A similar experiment can be made with actinium emanation. In this case the activity along the wire falls to half value for about every half centimetre. 


\section{CHAPTER VIII.}

\section{STANDARD MEASUREMENTS.}

\section{\$ 58. Radium Standards.}

The simplest way of defining the quantity of a radio-active substance is in terms of its mass; but it is only in the case of substances of long life, such as thorium, uranium and radium, that sufficient quantities accumulate to be weighable even by the most sensitive balance. With radium the quantities of material to be dealt with are frequently so small that its estimation is seldom made by the balance; moreover, to apply the method the radium salt must be pure, and this is often not the case. On this account primary standards containing known quantities of a pure radium salt have been constructed, and the determination of the radium content of other samples is then referred to the standards by a comparison of the activities of the sample and standard. The comparison is, whenever possible, made by means of $\gamma$ rays.

The question of establishing a standard was discussed at the International Congress of Radiology held at Brussels in 1910, and an international standard has since been prepared and deposited at Paris in the Bureau International des Poids et Mesures. The standard was prepared by taking a known weight of radium chloride separated by Mme. Curie for the determination of the atomic weight of radium. From the known chemical composition of the salt the quantity of radium present could be calculated.

The primary standard at Paris contains 21.99 mgrs. of radium chloride sealed in a glass tube $1.45 \mathrm{~mm}$. wide and $3.2 \mathrm{cms}$. long. The thickness of the walls of the tube is $0.27 \mathrm{~mm}$., so that the absorption of the $\gamma$ rays by the tube is 
small. Another primary standard containing $31 \cdot 17$ mgrs. of radium chloride has been deposited as a reserve standard at Vienna, and a comparison of the $\gamma$ radiation emitted by the two independent standards has shown them to agree with each other to within one-fifth of one per cent. Both these standards are free from mesothorium, since they have been prepared from Joachimsthal pitchblende, which contains no thorium. Standards must not be made of radium extracted from minerals containing thorium, since such preparations invariably contain mesothorium, which emits $\gamma$ rays and is inseparable from the radium by any known method. Uncertainty then arises as to the fractions of the $\gamma$ radiation due to the radium and mesothorium respectively; moreover, the activity of the preparation varies appreciably for a number of years after separation from the mineral on account of the decay of the mesothorium and the initial growth of thorium $D$.

Having established a primary standard, any sample of radium can be compared with it, and used as a secondary standard. For the construction of a secondary standard the same precautions must be taken as with the primary standard. A secondary standard containing one or two milligrams of radium chloride is sufficient for many purposes. For special investigations, such as the determination of the radium content of minerals, a very small standard is required. Such a standard can be prepared as described in $\$ 62$.

The amount of mesothorium in a preparation of that substance is usually expressed in terms of radium by comparing its $\boldsymbol{\gamma}$-ray activity with that of a radium standard. Thus a quantity of mesothorium giving, directly after preparation, the same $\gamma$-ray activity as a milligram of radium is sold as a milligram of mesothorium. The comparison is, however, arbitrary. For, in the first place, the penetrating power of the $\gamma$ rays from mesothorium is not the same as that of the radiation from radium, so that the comparison depends on the thickness of the lead screen used to cut off the $\beta$ rays. In the second place, it is impossible to obtain mesothorium preparations free from radium. 


\section{$\$ 59$. Comparison of Quantities of Radium by a $\gamma$-ray Electro- scope.}

The standardisation of large quantities of radium is almost invariably made by Rutherford's method of comparing the $\gamma$-ray activity of the sample under investigation with that of a known quantity of radium. Now radium itself emits no $\gamma$ rays, nor do the next three products which follow it in the radio-active series, except radium $B$, which has recently been shown to emit soft $\gamma$ rays; $*$ the penetrating $\gamma$ radiation of the radium is therefore entirely due to the fourth product, radium $C$, present in the radium salt, and it is necessary that the radium which must be hermetically sealed in a glass tube should be left for some weeks to come into equilibrium with its disintegration products before a comparison can be made. If the radium has been enclosed in a tube for at least a month, the radium $C$ will have reached its equilibrium value, and will be present in an amount proportional to the quantity of radium from which it is formed; the $\gamma$ radiation from the radium $C$ will then give a measure of the quantity of radium contained in the tube.

To estimate a quantity of radium in terms of the standard, the $\boldsymbol{\gamma}$-ray activity of each preparation is successively measured with an electroscope under identical conditions. The leaks produced in the electroscope after correcting for the natural leak are proportional to the quantities of radium contained in the tubes, and thus give a direct comparison of their amounts: The method is applicable to quantities of radium greater than one-tenth of a milligram. For accurate comparisons, the two samples should contain as nearly as possible the same amounts of radium, but it is possible to compare two samples, one of which contains ten times as much radium as the other. It is impossible to make with accuracy a direct comparison of two quantities which are still more dissimilar in quantity. In order that the experiments should give accurate results, attention must be paid to the following points : .

(1) The standard radium preparation is placed at such a distance from a $\gamma$-ray electroscope that a conveniently measur-

* Moseley and Makower, Phil. Mag. 23. 302, 1912. 
able ionisation (about 10 divisions per minute) is produced. The standard is then replaced by the specimen to be measured and the leak produced in the electroscope again measured. The accuracy of the measurements should be tested by repeating the observations with the radium at a different distance from the electroscope and with absorbing screens of different thicknesses of lead. The ratio of activity of the two samples should in each case be the same. Measurements must not be made with the samples too near the electroscope, since a small displacement relative to the electroscope introduces a large error.

(2) The radiation entering the electroscope must be entirely of the $\gamma$-ray type; for the absorption of the $\gamma$ rays in the radio-active material and the containing vessel is small, and therefore any slight difference in the thickness of the layer of the radio-active matter and the walls of the vessel containing it is without effect. 'This is obviously not the case for $\beta$ rays, which can therefore not be used for comparing quantities of radium. To insure that no primary or secondary $\beta$ rays enter the electroscope, its walls should be made of lead $3 \mathrm{mms}$. thick. Also, the glass windows of the electroscope should be at least $4 \mathrm{mms}$. in thickness. Scattered primary $\gamma$ radiation will, however, always enter the electroscope, for which reason objects near the electroscope should not be shifted during a series of experiments.

(3) All radio-active preparations other than the sample to be measured should be removed to a great distance, so as not to affect the electroscope by their $\gamma$ radiation.

(4) 'The samples of radium to be compared must be hermetically sealed to prevent emanation from escaping, for if this precaution is not taken, the radium $C$ in the preparations will not be in equilibrium with the radium. Moreover, if even a trace of emanation escapes during an experiment, this may find its way into the electroscope and by its $\alpha$-ray activity completely vitiate the comparison.

(5) 'The two samples should if possible be enclosed in small tubes, so that they act as nearly as possible as point sources. Moreover, the walls of the tubes should be not much more 
than one-fifth of a millimetre thick, in order that the absorption of the $\gamma$ rays by the tube may be negligible. In case the tube has thicker walls, a correction can be introduced for the absorption of the rays assuming a coefficient of absorption $11 \mathrm{~cm}^{-1}$ for glass.

(6) If, as is occasionally the case, the radium has been prepared from a mineral containing thorium, serious errors may be introduced in determining a quantity of radium on account of the possible presence of mesothorium, which also emits $\gamma$ rays ( $\$ 58)$. There is no very simple way of determining whether mesothorium is mixed with the radium; but its presence can be detected by dissolving the salt and driving off the emanation. If the solution contains no mesothorium, the $\gamma$-ray activity will almost vanish after three hours; but this will not be the case if any mesothorium is present. On account of the risk of the loss of radium in the manipulations, the test should be applied only when there is definite reason to suspect the presence of mesothorium.

\section{$\$ 60$. Balance Method of Standardising Radium.}

When comparing quantities of radium by the $\gamma$-ray method, the chief source of uncertainty arises from the difficulty of knowing whether the current through the electroscope is saturated in every measurement. The error introduced on account of lack of saturation may be particularly serious when quantities of radium of very different activities are compared. A very delicate method which avoids this trouble and does away with the necessity of measuring the natural leak has been devised by Rutherford and Chadwick.* 'The method consists in balancing the ionisation current produced in an ionisation vessel by the $\gamma$ rays from the two specimens of radium in turn, against that produced in a second vessel by some uranium placed inside it. The ionisation in this vessel remains constant, while that in the other one is varied by altering the distance of the radium from it. When the ionisation currents have been balanced, the position of the radium on a scale gives a measure of the quantity present.

* Rutherford and Chadwick, Proc. Phys. Soc. Lond. 24. 141, 1912. 
The general arrangement of the apparatus is shown in figure 56. By adjusting the distance of the radium $R$ from the vessel $A$, the current produced by the $\gamma$ rays is balanced against that flowing through the gas in $B$, in which is placed the uranium oxide. The electrometer indicates whether a balance has been established. Observations are taken successively with a radium standard, and the radium preparation to be measured and the distances from the vessel $A$ at which balance occurs is noted in each case. Since, over a wide range of distance, the intensity of the radiation producing ionisation is inversely proportional to the square of the distance of the radium from the vessel $A$, the strengths of two sources can be compared. In the apparatus described by Rutherford

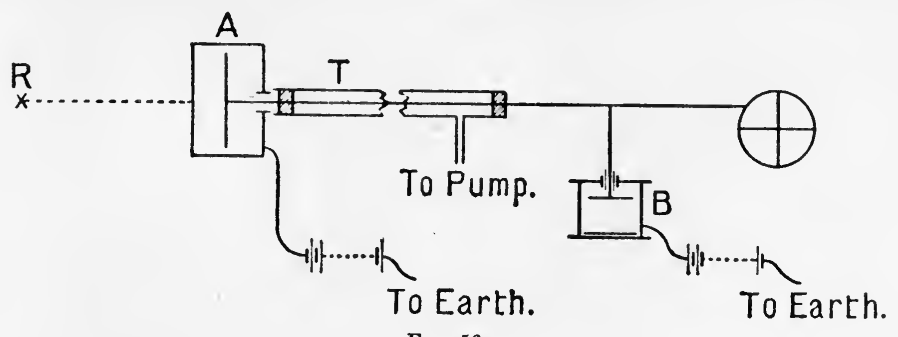

Fig, 56.

and Chadwick, the vessel $A$ consisted of a lead cylinder $2 \mathrm{cms}$. thick, $10 \mathrm{cms}$. long and of internal diameter $15 \mathrm{cms}$. One end of the cylinder was closed by a lead plate $2 \mathrm{cms}$. thick, and the other, through which the $\gamma$ rays entered, was one centimetre thick. The internal electrode consisted of a circular aluminium plate one millimetre thick and $12 \mathrm{cms}$. in diameter, and was insulated by a sulphur stopper with a guard ring. The connections to the electrometer were made by a wire passing through a metal tube $T$ several metres long, which was exhausted to prevent the air surrounding the wire being ionised by the $\gamma$ rays.

The vessel $B$ consisted of a brass cylinder $4 \mathrm{cms}$. high and of diameter $4 \mathrm{cms}$. A film of uranium oxide deposited by M'Coy's method was placed inside the vessel ( $\$ 30)$. Above the film was an iris diaphragm, the aperture of which could be varied from $5 \mathrm{~cm}$. to $3 \mathrm{cms}$, thus changing the ionisation 
over a large range. The base of the vessel was screwed to an ebonite block fastened to a lead block $1 \mathrm{~cm}$. thick. The vessel was protected from external $\gamma$ rays by a lead cylinder $6 \mathrm{cms}$. high and $3 \mathrm{cms}$. thick. The electrode was a circular copper plate.

To make the comparison of the two preparations of radium, it is necessary to take account of the absorption of the $\gamma$ rays by the air intervening between the sources and the vessel $A$. If $d$ is the thickness of the lead plate and $r$ the distance of the source from the front surface of the vessel, then if $\mu_{1}$ is the absorption coefficient of the $\gamma$ rays in lead and $\mu$ the coefficient in air, the intensity of the radiation in the ionisation vessel is proportional to

$$
\frac{e^{-\mu_{1} d} e^{-\mu r}}{r(r+a)}
$$

where $a$ represents the depth of the ionisation vessel, which must be small compared with $r$. Hence, if $S$ milligrams, placed at a distance $r_{1}$ from the ionising vessel, produce the same ionisation as $R$ milligrams placed at a distance $r_{2}$, it follows, since the absorption by the lead plate is the same in both cases, that

or

$$
\begin{aligned}
\frac{R e^{-\mu r_{2}}}{r_{2}\left(r_{2}+a\right)} & =\frac{S e^{-\mu r_{1}}}{r_{1}\left(r_{1}+a\right)} \ldots \ldots \ldots . . . \\
\frac{R}{S} & =\frac{r_{2}}{r_{1}} \cdot \frac{r_{2}+a}{r_{1}+a} e^{-\mu\left(r_{1}-r_{2}\right)} .
\end{aligned}
$$

If the $\gamma$ rays pass through $1 \mathrm{~cm}$. of lead before entering the ionisation vessel, $\mu=0.000060(\mathrm{~cm} \text {. })^{-1}$ for air at normal temperature and pressure.

The method as described above can be used to measure quantities of radium greater than one milligram. If smaller quantities have to be compared, the preparations have to be brought so near to the ionisation vessel that the inverse square law no longer holds. The variation of the ionisation with the distance of the preparation from the ionisation vessel must then be determined experimentally.

\section{$\$ 61$. Quantitative Estimation of Radium Emanation.}

The method given above of comparing two samples of radium can be used for measuring the quantity of radium 
emanation contained in a tube. Since the period of the emanation is long compared with that of the active deposit, the quantity of radium $C$ in equilibrium with the emanation is very nearly the same whether the emanation is in contact with its equilibrium quantity of radium or not. Thus, the $\gamma$-ray activity of a tube containing emanation separated from its radium will be very nearly the same as if the quantity of radium in equilibrium with the amount of emanation present were actually contained in the tube.

The quantity of emanation in equilibrium with one gram of radium is known as a curie of emanation, and one-thousandth part of it as a millicurie. Similarly the quantity of radium $A$, radium $B$ and radium $C$ in equilibrium with one gram of radium are known as one curie of radium $A$, radium $B$ or radium $C$ respectively. Thus, if the $\gamma$-ray activity of a tube containing radium emanation is compared with that of a standard tube of radium, the quantity of emanation present expressed in curies can be directly determined.

The method of procedure is exactly similar to that described above for comparing the amounts of radium contained in two tubes; but to obtain an accurate determination of a quantity of emanation a correction must be applied. For consider a tube filled with emanation free from its products of disintegration. Since the emanation itself emits no $\gamma$ rays, the tube will at first exhibit no activity; but as radium $B$ and radium $C$ are formed, $\gamma$ rays will appear, and the activity will continue to rise, reaching a maximum after about four hours, and ultimately decay with the period of the emanation. The emanation is then apparently in equilibrium with its products of disintegration; but it will be seen that the state is not one of true equilibrium, in which the numbers of atoms of successive products disintegrating per second are equal. For in the time which elapses for the apparent equilibrium to be established the emanation will have decayed, with the result that the numbers of atoms of radium $B$ and radium $C$ disintegrating per second will be somewhat in excess of the number of atoms of emanation breaking up per second at the same moment. The name transient equilibrium has been 
given to this state of apparent equilibrium, which exists whenever the life of a product is not negligibly short compared with that of the preceding substance which controls the decay.

It is easy to calculate the correction which must be applied when estimating quantities of emanation by comparison with a radium standard, in which true equilibrium exists between the disintegration products. For let $\lambda_{1}, \lambda_{2}, \lambda_{3}$ and $\lambda_{4}$ be the disintegration constants of the emanation and its successive products respectively. Then, if the number of atoms of emanation breaking up per second at the beginning is taken as unity, the number of atoms of radium $C$ decaying per second at any subsequent moment is given by

$$
\begin{aligned}
N= & \lambda_{2} \lambda_{3} \lambda_{4}\left[\frac{e^{-\lambda_{1} t}}{\left(\lambda_{2}-\lambda_{1}\right)\left(\lambda_{3}-\lambda_{1}\right)\left(\lambda_{4}-\lambda_{1}\right)}+\frac{e^{-\lambda_{2} t}}{\left(\lambda_{1}-\lambda_{2}\right)\left(\lambda_{3}-\lambda_{2}\right)\left(\lambda_{4}-\lambda_{2}\right)}\right. \\
& \left.+\frac{e^{-\lambda_{3} t}}{\left(\lambda_{1}-\lambda_{3}\right)\left(\lambda_{2}-\lambda_{3}\right)\left(\lambda_{4}-\lambda_{3}\right)}+\frac{e^{-\lambda_{4} t}}{\left(\lambda_{1}-\lambda_{4}\right)\left(\lambda_{2}-\lambda_{4}\right)\left(\lambda_{3}-\lambda_{4}\right)}\right] .(59)
\end{aligned}
$$

After the lapse of some hours the exponential terms involving $\lambda_{2}, \lambda_{3}$ and $\lambda_{4}$ become negligible, and therefore the number of atoms of radium $C$ decaying per second is given by

$$
N^{\prime}=\frac{\lambda_{2} \lambda_{3} \lambda_{4} e^{-\lambda_{1} t}}{\left(\lambda_{2}-\lambda_{1}\right)\left(\lambda_{3}-\lambda_{1}\right)\left(\lambda_{4}-\lambda_{1}\right)}
$$

But in the case of true equilibrium the number of atoms of radium $C$ breaking up is given by $e^{-\lambda_{1} t}$. Hence the ratio of the number of atoms of radium $C$ actually decaying to the number which would decay, had true equilibrium been established, is given by

$$
\frac{\lambda_{2} \lambda_{3} \lambda_{4}}{\left(\lambda_{2}-\lambda_{1}\right)\left(\lambda_{3}-\lambda_{1}\right)\left(\lambda_{4}-\lambda_{1}\right)}
$$

By inserting the known values of $\lambda_{1}, \lambda_{2}, \lambda_{3}$ and $\lambda_{4}$, this expression can be shown to be equal to 1.0089. Thus the observed quantity of emanation is 0.89 per cent. in excess of the actual amount present, and this is the correction to be applied when the measurements are made with the hard $\gamma$ rays left after penetrating $2 \mathrm{cms}$. of lead. If, however, the measurements are made through $3 \mathrm{mms}$. of lead, as is sometimes the case, the $\gamma$ radiation is due not only to 
radium $C$, but also to radium $B$. In this case the correction is somewhat smaller, and can be shown to be about 0.85 per cent. (see also Appendix II.).

\section{$\$ 62$. Determination of small Quantities of Radium (Emanation method).}

The $\gamma$-ray method of measuring quantities of radium can be used only with comparatively large quantities of active material. With $0.1 \mathrm{mg}$. of radium the measurements become troublesome, and with smaller quantities the method fails to give good results. A very sensitive method of measuring small quantities of radium depends upon the determination of the quantity of emanation associated with the radium. The method, which has been worked out in detail by Boltwood,* consists in introducing the emanation into an electroscope and comparing its ionising power with that produced by the emanation from a standard quantity of radium. The standard and sample of radium to be compared must be in solution, from which the emanation can be quantitatively removed by boiling. The sensitiveness of the method is very great, since the comparison is based on $\alpha$-ray instead of $\boldsymbol{\gamma}$-ray measurements.

In order to prepare a small standard, a quantity of radium is measured accurately by the $\boldsymbol{\gamma}$-ray method and then dissolved in dilute hydrochloric acid. The solution is diluted with weak acid and a small fraction drawn off. This is again diluted, and the operation is repeated until a solution is obtained containing about $10^{-5} \mathrm{mgr}$. of radium per cubic centimetre. One cubic centimetre of the solution is then taken and placed in a carefully cleaned distilling flask of about 250 c.c. capacity, and the volume increased to about 100 c.c. by adding dilute hydrochloric acid. The precautions necessary to keep the radium in solution have already been discussed (\$44). The solution is boiled to drive off all the emanation, and the flask is then sealed. The amount of emanation which collects in a definite time, reckoned from the moment of sealing the flask, can easily be calculated in terms of the equilibrium quantity.

$$
\text { * Boltwood, Phil. Mag. 9. 599, } 1905 .
$$

R.-A. 
If very great accuracy is not required, a standard solution can be made by dissolving in nitric acid about 50 mgrs. of pitchblende of known composition. The strength of the solution can be deduced from the known composition of the mineral; for since radium is a disintegration product of uranium, the amount of radium present in the mineral is proportional to its uranium content, $3.4 \times 10^{-7} \mathrm{gr}$. of radium being in equilibrium with one gram of uranium. Thus, for instance, $1 \mathrm{gr}$. of Joachimsthal pitchblende contains $0.617 \mathrm{gr}$. of uranium, and therefore $2 \cdot 1 \times 10^{-4} \mathrm{mgr}$. of radium.*

To prepare a standard solution, one gram of pure pitchblende is dissolved in nitric acid. A small insoluble residue remains, which may contain part of the radium. The solution is filtered off and the residue washed with hot hydrochloric acid to remove the radium salts adhering to it. The solution which then contains the whole of the radium is evaporated to dryness on a water bath and the residue dissolved in hydrochloric acid. The solution is diluted and a twentieth part taken. This quantity contains $1 \cdot 05 \times 10^{-5} \mathrm{mgr}$. of radium.

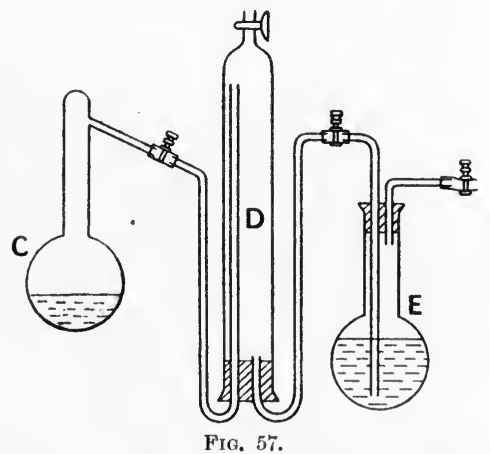

The transference of the emanation from the radium solution to the electroscope is made by means of the apparatus shown in figure 57. $\dagger$ The distilling flask $C$, containing the radium solution, is connected to the gas burette $D$. The emanation is collected in it over hot water, since cold water dissolves the emanation to an appreciable extent. At the beginning of an experiment, the burette is filled with hot water

\footnotetext{
* Boltwood, Phil. Mag. 9. 599, 1905.
}

† Boltwood, loc. cit. 
forced into it from the flask $E$, which is heated. The solution containing the radium is then boiled so as to drive the air above the solution into the burette, and the boiling is continued for about five minutes to ensure that all the emanation is evolved and carried into the burette by the steam generated. The burette is then closed and the flask sealed-off. The solution is then put aside for emanation again to collect for a subsequent experiment. From the burette the gas is sucked into the electroscope, with which its activity is measured. To do this the electroscope is exhausted with a water pump,* and the emanation then allowed to flow through a drying tube into it from the burette. The burette is then detached from the electroscope and a stream of dry air passed through the connecting tubes into the electroscope to sweep out any emanation remaining in them, and to establish atmospheric pressure inside the electroscope. The electroscope is then closed and left for four hours to allow the emanation to produce its equilibrium quantity of radium $A$, radium $B$ and radium $C$. In the meantime, the electroscope should be kept charged to ensure a definite distribution of the active deposit between the electrodes, which then remains unaltered when measurements are being taken. It is best to charge the leaf negatively, so as to concentrate the active deposit on the central rod. On account of the intense field near the central electrode, saturation is obtained more easily than if the active deposit is driven to the walls of the electroscope. The rate of leak of the electroscope gives a measure of the activity of the radium solution. The emanation should not be left in the electroscope longer than is essential for the measurements; otherwise the radium $F$ formed will gradually increase the natural leak.

* If an oil pump is used instead of a water pump, great care must be taken not to use a pump which has been employed in experiments with large quantities of emanation; for the emanation dissolves in the oil and may be subsequently given off and enter the electroscope. The measurements may then be entirely vitiated. For the same reason care must be taken when transferring emanation not to use mercury contaminated with active matter. 
The process described above is repeated with the solution to be standardised. In case the radium content of this solution is large compared with that of the standard, the solution should be boiled to drive off all the emanation and then sealed up; after a definite time, depending on the strength of the solution, an amount of emanation will have collected which is approximately equal to the equilibrium amount in the standard. The emanation should then be transferred to the electroscope as described above, and its amount measured. From a knowledge of the quantity $Q$ of emanation which has collected in the solution in the definite time selected, it is possible to calculate the equilibrium quantity $Q_{0}$ corresponding to the radium in solution from the equation

$$
Q=Q_{0}\left(1-e^{-\lambda t}\right)
$$

where $t$ is the time during which the emanation has accumulated and $\lambda$ is the decay constant of the emanation.

\section{$\$ 63$. Activity of Minerals and Mineral Waters.}

The method described in $\S 62$ can also be used for measuring the radio-activity of mineral waters and minerals. Consider first the case of mineral waters, where the activity is as a rule due to radium emanation brought up in solution from radium deposits underground or sometimes to small quantities of radium dissolved in the spring water. It is easy to distinguish between these two possibilities, for in the former case the water will remain permanently inactive after removing the emanation, whereas in the latter case the activity will gradually reappear after boiling off the emanation. In either case the quantity of emanation dissolved in the water can be measured by introducing it into an emanation electroscope which has been standardised with a radium solution of known strength.

The radium content can easily be determined in the case of minerals, such as for instance pitchblende, carnotite and autunite, which dissolve in nitric acid. The solution of the mineral is boiled to drive off all the radium emanation in solution, and then allowed to stand for a known time for emanation to be regenerated. The emanation is then again boiled off, collected and its amount determined. In 
the case of insoluble minerals, special chemical methods must be adopted to bring them into solution, after which the radium content can be found. Another method of extracting the emanation contained in radio-active minerals has been devised by Joly. The mineral is mixed with sodium and potassium carbonates and raised to a bright red heat in an electric furnace. The gases evolved which contain the emanation are collected and transferred to an electroscope. For further details the reader is referred to the original paper of Joly.*

\section{$\$ 64$. Standardisation by the Method of Scintillations.}

The quantitative estimation of radio-active products emitting $\alpha$ rays can be effected by counting the scintillations produced by the $\alpha$ particles on a zinc sulphide screen. In the radium series, it is known that one curie of any product emits $3.4 \times 10^{10} a$ particles per second, so that the number of $\alpha$ particles emitted gives a means of determining the quantity of the product present.

A determination of the number of $\alpha$ particles emitted can be made by counting the number of scintillations produced on a definite area of a zinc sulphide screen placed at a measured distance from the source of radiation. If the screen is uniformly and sufficiently thickly coated with zinc sulphide crystals, almost every $\alpha$ particle striking it produces a scintillation. Now, since the $\alpha$ particles are emitted equally in all directions, the total number $Q$ of $\alpha$ particles emitted by the source per second will be given by the equation,

$$
Q=\frac{4 \pi r^{2} N}{A}
$$

where $N$ is the number of $\alpha$ particles striking the screen per second, $A$ is the area of the screen and $r$ the distance between the source and screen. The method of counting the scintillations has already been given in $\$ 33$. The experiment can be carried out by means of the apparatus shown in figure 29. The active plate $S$ is mounted at the end of a glass rod $D$, which can slide through an airtight rubber joint. 
It is introduced into the tube $A$, which should be about $3 \mathrm{cms}$. in diameter, through the ground glass joint $C$, and its distance from the zinc sulphide screen $R$ is adjusted so that about 40 scintillations appear on it per minute when the tube $A$ is evacuated. It will be found that even with a constant source of radiation, the number of scintillations appearing per minute on the screen varies between wide limits (\$37). In order to get an average of sufficient accuracy at least a thousand scintillations must be counted. The total number of $\alpha$ particles emitted by the source per second can then be calculated from equation (62) if the distance from the source to the screen is accurately measured and the area of observation on the screen is known. The latter quantity is deduced from the diameter of the field of view of the microscope, which can be determined by focussing on a graduated scale. In order to check the accuracy of the measurements, the experiment should be repeated with different distances between the source of radiation and the screen.

The following precautions must be taken to obtain accurate results :

(1) The screen must be tested from time to time to see whether it is itself inactive and whether it is receiving particles from sources other than the plate $S$. This can be done by removing the plate, or by admitting into the apparatus sufficient air to stop the $\alpha$ particles from the plate reaching the screen. The scintillations should then completely cease, but if not, allowance must be made for the number remaining when computing the number of $\alpha$ particles from the source.

(2) It is important that every $\alpha$ particle reaching the screen should strike a zinc sulphide crystal and so produce a scintillation. The plate on which the zinc sulphide is deposited must therefore be completely, but not too thickly, covered with phosphorescent material. The ratio of the number of scintillations appearing on the screen to the number of $\alpha$ particles striking it is called the efficiency of the screen, and if, as is usual, the efficiency is only 80 or 90 per cent., allowance must be made for this fact. 
Before using a screen its efficiency should be tested by counting the scintillations from a known quantity of active material such as the active deposit from radium, the activity of which has been measured in the usual way by the $\gamma$-ray method. It must, however, be pointed out that if the source is active enough to be measured by $\gamma$ rays, the number of $a$ particles emitted will be so great that, to permit accurate counting, the screen must be placed several metres away. Perfect efficiency can be obtained by observing the scintillations on a carefully polished fragment of diamond; but the scintillations are much fainter than on zinc sulphide, and their intensity varies greatly for different specimens.*

(3) Care must be taken that no $\alpha$ particles reach the screen from the active source by reflection from the walls of the tube. If the distance from source to screen is not much greater than the diameter of the tube, the error introduced will be very small, as the probability of $\alpha$ particles being scattered through large angles is minute. But when the distance from source to screen is large compared with the diameter of the tube, scattering from the walls of the tube through small angles will increase the number of $a$ particles received on the screen. In this case diaphragms should be fixed in the tube to prevent scattered radiation reaching the screen.

* Regener, Sitzungsberichte der preussischen Akademie der Wissenschaften, 38. 948, 1909. 


\section{CHAPTER IX.}

THE SEPARATION OF RADIO-ACTIVE SUBSTANCES.

\section{$\$ 65$. General Methods of Separation.}

It has already been pointed out that radio-active bodies can sometimes be separated from each other by the method of recoil $(\$ 46)$. The method is very convenient to obtain large yields of radium $B$, actinium $D$ and thorium $D$ in a state of great purity. But the method applies only to a limited number of cases, and other means of separation have frequently to be employed. In some instances it is possible to isolate products by distillation, for the temperatures of volatilisation of different members of the radio-active series are not the same; but the method, though sometimes applicable, is complicated by the fact that the volatilisation point of a product depends upon the nature of the surface on which the material is deposited.* Another method which can be used is that of electrolysis, which has been worked out in detail by von Hevesy. $\dot{\dagger}$ Radio-active substances may also be separated by precipitating the material which is usually present in minute quantity in presence of an element resembling it in chemical properties. In these circumstances the radio-active matter is carried down with the precipitate, and is thus separated.

In this chapter some important methods of separation are given. For a fuller account of the chemical properties of the radio-active elements, the reader is referred to other text-books. $\ddagger$

* Schrader, Phil. Mag. 24. 125, 1912.

† von Hevesy, Phil. Mag. 23. 628, 1912.

$\ddagger$ Cf. Soddy, The Chemistry of the Radio-Elements. Longmans, Green \& Co., 1911. 
The manipulations described in this chapter should be carried out in a room specially set aside for chemical work; this diminishes the risk of contaminating measuring instruments with radio-active matter. Since it is impossible to remove the last traces of radio-active substances from the glass apparatus used in the chemical processes, it is essential to keep separate sets of beakers for the different separations.

\section{$\$ 66$. Separation of Uranium $\mathrm{X}$ from Uranium.}

There are several methods of separating uranium $X$ from uranium, two of which are given below.

Ferric-hydroxide Method. The best method of separating uranium $X$ is by the process originally used by Crookes.* A solution of uranium nitrate is obtained either by dissolving a few grams of uranyl nitrate in water or by dissolving. uranium oxide in dilute nitric acid. To the solution are added about 20 mgrs. of ferric chloride dissolved in water. 'The solution, which is then diluted to a volume of at least 100 c.c., is raised to the boiling point, and a strong solution of ammonia containing ammonium carbonate added slowly. Meanwhile the solution is agitated till the uranium hydroxide, precipitated by the ammonia, is redissolved in the ammonium carbonate, whereas the uranium $X$ and the ferric hydroxide remain precipitated. The uranium $X$ is filtered off with the ferric hydroxide, washed with ammonium carbonate to get rid of the last traces of uranium hydroxide, and then dried. By this method 95 per cent. of the uranium $X$ are separated in a single operation.

Separation of Uranium $X$ by Animal Charcoal. Another method of separating uranium $X$ consists in adding to a dilute solution of uranium nitrate, animal charcoal or freshly prepared soot obtained by burning camphor. The solution is boiled for about half an hour, after which the soot or charcoal is filtered off. The uranium $X$ is separated with the soot by which it is absorbed. 'To concentrate the uranium $X$ the soot can then be removed by burning.

* Crookes, Proc. Roy. Soc. A. 66. 409, 1900. 


\section{$\$ 67$. Separation of Radium $\mathrm{C}$ from the Active Deposit of Radium.}

'To obtain radium $C$ free from radium $A$ and radium $B$, the method of von Lerch $*$ is generally used. The active deposit of radium is obtained on a platinum surface by exposure for some hours in an electric field to radium emanation in the usual manner; or radium emanation is allowed to stand for some hours in a glass tube to produce the active deposit on the walls, when the emanation is transferred to another vessel. The platinum surface or the tube is then washed with alcohol to remove traces of emanation adhering to it, and the active deposit is dissolved in hot and strong hydrochloric acid. The solution is diluted and a nickel plate immersed in it. If the solution is agitated, within a few minutes the greater part of the radium $C$ is deposited on the plate by electro-chemical action. The plate is then thoroughly washed with hot water to remove the solution adhering to it.

In carrying out the separation, time must be allowed for all the radium $A$ to decay before immersing the nickel plate in solution; for radium $A$, if present, will be deposited on the nickel with the radium $C$ and in decaying produce radium $B$. If an interval of 20 minutes is allowed, the radium $A$ collected by exposure to the emanation will have decayed to about one per cent. of its original activity, whereas the amount of radium $C$ will be only slightly diminished. Since usually the activity is only required on one side of the nickel plate, the other side should be covered with an insoluble varnish which prevents the deposition of radium $C$ on that side.

\section{$\$ 68$. Separation of Radium $D$ and Radium $\mathbf{E}$.}

A radium preparation which has been allowed to stand for some years without removing the emanation will contain appreciable quantities of radium $D$, radium $E$ and radium $F$. Considerable activities can be obtained by separating these elements from old preparations of radium containing only $\cdot 01$ to $\cdot 1 \mathrm{mg}$. of radium.

Radium $D$ closely resembles lead in chemical properties, and can be separated from solutions with that element. It * von Lerch, Ann. der Phys. 20. 345, 1906. 
is on that account that the element was first named radiolead. The radium preparation is dissolved in hydrochloric acid, and a trace of lead chloride added to the solution. The lead is precipitated with sulphuretted hydrogen, which at the same time brings down radium $D$. Since, in this process, some radium usually comes down, the precipitate is filtered off, redissolved in strong nitric acid, and the lead reprecipitated with sulphuretted hydrogen. The lead sulphide which contains the radium $D$ is tiltered off. Radium $D$ itself emits only very soft $\beta$ rays, but radium $E$ will at once begin to grow, and at the end of a few weeks will have come into radio-active equilibrium with the radium $D$. The precipitate will then act as an almost constant source of $\beta$ radiation. After some months polonium will have been formed in appreciable quantity, and the precipitate will then also emit $\alpha$ rays.

Instead of separating radium $D$ from a radium preparation, it is preferable to grow radium $D$ from the emanation. For this purpose at least 10 millicuries of emanation should be collected and allowed to decay in a closed tube. The active deposit formed on the walls changes into radium $D$, and after some weeks the whole of the emanation will have been converted into radium $D$. This is dissolved in concentrated nitric acid, by evaporating which the radium $D$ can be deposited on a glass or platinum surface.

Radium $E$ can easily be separated by immersing a nickel plate in a solution of radium $D$, obtained by dissolving the active deposit from radium, or by electrolytic deposition on a silver cathode with a current of density about one milliampere per square centimetre. The solution should be free from polonium, for this, if present, will also be deposited. The presence of lead vitiates the method, so that it cannot be employed to separate radium $E$ from the lead residues from pitchblende, unless the greater part of the lead is first separated.*

\section{$\$ 69$. Separation of Radium F (Polonium).}

Polonium was first separated from pitchblende with bismuth, with which it is precipitated by sulphuretted hydrogen.

* Meitner, Phys. Zeilschr. 12. 1094, 1911. 
It is, however, much simpler to obtain it from a radium $D$ solution in which polonium has been allowed to accumulate. This can be done by immersing a carefully polished plate of copper or bismuth in the solution for about half an hour. The solution should be not too strongly acid; for otherwise the surface of the plate may be attacked. One side of the plate may be coated with varnish to prevent deposition on that side; or the plate may be attached with wax to the end of a glass tube, and the solution poured into it.

In the same manner the polonium can be separated directly from a solution of radium. In the process some of the quickly decaying products of the radium may be deposited, but these will disappear in the course of a few hours, leaving pure polonium.

\section{$\$ 70$. Separation of Actinium $X$.}

To separate actinium $X$ from a preparation of actinium, the latter is dissolved in the least possible amount of hydrochloric acid,diluted to about 20 c.c., and the actinium precipitated from the solution with excess of pure ammonia: the actinium $X$ remains in solution. It is important that the ammonia should contain no carbonate, since actinium salts are soluble in ammonium carbonate. In order to be sure that the actinium has been completely precipitated, the solution must be kept boiling for about half an hour and then left to stand for half a day. The actinium is filtered off and the solution evaporated to dryness to drive off the ammonium chloride. The minute residue left contains the actinium $X$. The separation is practically quantitative.

\section{$\$ 71$. Separation of Radio-actinium.}

It is troublesome to make a quantitative separation of radio-actinium from a solution of actinium. Strongly active preparations of radio-actinium can, however, be obtained by the following methods*:

(1) The actinium preparation is dissolved in hydrochloric acid and the actinium $X$ separated from it as explained in $\$ 70$. The radio-actinium remains in the precipitated

* Hahn, Phys. Zeitschr. 7. 855, 1906. 
actinium, which is redissolved in a very small amount of hot concentrated hydrochloric acid. The solution is then diluted to about 20 c.c., and a drop of a solution of sodium thiosulphate is added and the solution boiled to precipitate sulphur in a finely divided state. The radio-actinium is precipitated with the sulphur, with which it can be removed by filtration. On-burning the sulphur and the filter paper on which it is collected, a small quantity of highly active material is left containing radio-actinium free from actinium and actinium $X$.

(2) Another way of effecting the separation is by fractional precipitation with ammonia. Instead of precipitating the whole of the actinium from the solution, only enough ammonia is added to bring down a fraction of the actinium. The precipitate contains an excess of radio-actinium.

The precipitate is filtered off, redissolved in hydrochloric acid and partially reprecipitated with ammonia. By repeating this process several times, a small amount of precipitate is finally obtained, which contains radio-actinium almost free from actinium and actinium $X$.

\section{$\$ 72$. Separation of Actinium C.}

Actinium $C$ is isolated by a method similar to that employed in the separation of radium $C(\$ 67)$. The active deposit is first collected on a platinum surface by exposure to the emanation in an electric field in the usual manner. The platinum plate is removed and placed in boiling hydrochloric acid, which dissolves off the active deposit. The solution is then partially neutralised so as to leave it only slightly acid, and a nickel plate placed in it for one or two minutes. The actinium $C$ will be deposited on the plate, which is then removed and washed with water. The plate will retain its activity only for a short time, since actinium $C$ decays to half value in $2 \cdot 15$ minutes.

\section{$\$ 73$. Separation of Thorium $\mathrm{X}$ and Mesothorium.}

It is impossible to separate radio-thorium from thorium or mesothorium from thorium $X$, although the first two substances can easily be separated from the second two. 
Thorium $X$ can be separated either from thorium salts or from the preparations of radio-thorium which have been placed on the market. The radio-thorium contained in these preparations is grown from mesothorium; this is the only way of obtaining radio-thorium free from thorium.

To separate thorium $X$ from thorium a few grams of thorium nitrate are dissolved in water and the solution diluted to about 100 c.c. Ammonia is then added in excess to the hot solution and the thorium thereby precipitated as hydroxide. Thorium $X$ and mesothorium are left in solution, while the radio-thorium is precipitated with the thorium. The solution is separated from the precipitate by filtration and boiled down to dryness to drive off the ammonium salts. The residue left contains the thorium $X$ and any mesothorium originally present in the thorium.

If one gram of thorium nitrate is taken, the activity of the thorium $X$ separated will be sufficient to produce considerable ionisation in an $\alpha$-ray electroscope. The $\alpha$-ray activity of the thorium $X$ will decay with a period of $3 \cdot 7$ days. If, however, mesothorium is present, the activity will ultimately slowly rise again on account of the formation of radio-thorium and its subsequent products.

Owing to its short period, the amount of thorium $X$ is always proportional to the quantity of radio-thorium present in the preparation. Hence, to obtain large quantities of thorium $X$, it is advantageous to separate the thorium $X$ from the concentrated radio-thorium preparations. The separation can be effected by the same process as that described above.

In thorium salts the amount of thorium $X$ present depends on the time which has elapsed since preparing it from the mineral. For in the process of preparing the thorium salt and in all subsequent separations of thorium $X$, all the mesothorium present is also separated. On account of the absence of its parent substance mesothorium, the amount of radiothorium will diminish and with it its equilibrium quantity of thorium $X$. If frequent precipitations are made extending over a period of many years, the radiothorium originally 
present will have completely decayed without being regenerated. The solution will then cease to yield thorium $X$.

\section{$\$ 74$. Separation of Thorium B and Thorium C.}

To obtain thorium $C$, the active deposit of thorium is collected on a platinum foil and then dissolved in hydrochloric acid. Thorium $C$ is then deposited on a nickel plate by the same method as that employed for separating radium $C$ and actinium $C$ (\$ 67 and 72 ). Thorium $B$ and thorium $D$ remain in solution.

In order to obtain pure thorium $B$ on a plate, thorium $C$ is first removed from the solution by von Lerch's method, and immediately afterwards the hot solution is electrolysed for some minutes, using a platinum cathode. The current density should be about one milliampere per square centimetre.

\section{$\$ 75$. Separation of Emanation from Radium Solutions.}

It is often advantageous to use as a source of radiation radium emanation instead of radium itself ; for with emanation the amount of radio-active material involved is so small that the absorption by the matter even of the $a$ particles is negligible; moreover, the accidental loss of radium during manipulations is in this way avoided.

It is desirable, even with small quantities, to keep the radium in solution, so that the emanation can be withdrawn when required. The emanation evolved is mixed with other gases, so that for many purposes it is necessary to purify it. The separation of radium emanation and its subsequent purification can be effected by methods developed by Rutherford. ${ }^{*}$ In order to separate the emanation, the radium should be kept dissolved in hydrochloric acid, as explained in $\$ 44$. The solution, the volume of which should be about 50 c.c., is placed in the bulb $A$ (fig. 58) of about 100 c.c. capacity mounted inside a strong glass vessel $F$, which serves to catch the solution in case of an accidental breakage of the flask. When working with strong solutions, this vessel is surrounded by thick lead to cut off the $\gamma$ rays, which otherwise may interfere

* Rutherford, Phil. Mag. 16. 300, 1908. 
with radio-active measurements being made in neighbouring rooms.

The bulb $A$ is provided with a long neck leading to the tap $C$, and is permanently attached to a Toepler pump, by which the whole system is exhausted. Under the reduced pressure the radium solution boils and expels the emanation, which is carried into the pump with the other gases, which

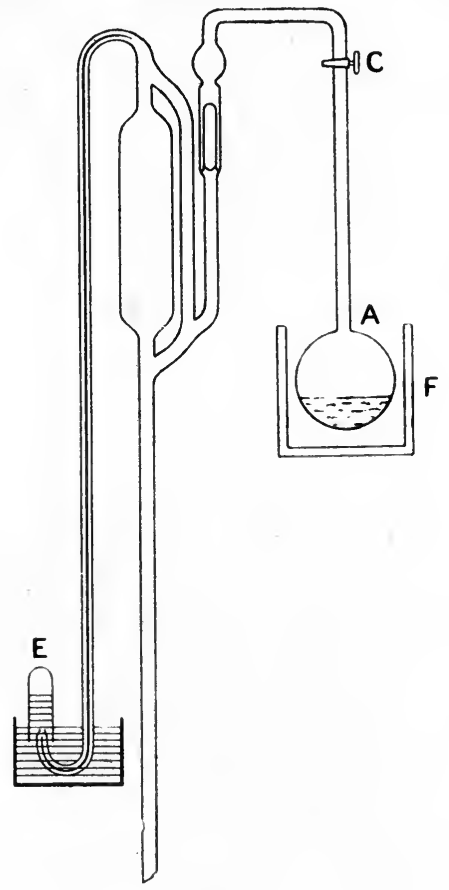

Fir. 58.

are then pumped into the tube $E$. When evacuated, the tap $C$ is closed and the solution allowed to stand for emanation again to be produced. At the end of four days, half the equilibrium amount will have again accumulated. Although previously evacuated, the space above the radium solution will contain a certain amount of gas besides the emanation, for under the influence of the radiation the water is decomposed with the evolution of oxygen and hydrogen, which then serve 
to sweep the emanation from the flask $A$ into the pump. The amount of gas accumulated depends upon the amount of radium present, and on the time for which the solution has been standing. A solution containing one milligram of radium yields about 01 c.c. of gas per day. After transference into the pump the gases are forced into the tube $E$, and the pumping is continued until all the gases have been removed from the bulb. When this is the case the solution begins gently to boil under the reduced pressure; the tap $C$ is then closed. No emanation should be allowed to stand in the pump, for in its presence the mercury is oxidised and the glass also soon becomes coloured. The pump is kept dry by phosphorus pentoxide contained in a side tube.

The gases pumped off from the radium solution consist for the most part of oxygen and hydrogen derived from the decomposition of the water in the solution. In addition there is, however, usually present an appreciable amount of carbon dioxide produced by the action of the emanation on the grease used to lubricate the stop-cocks. The separation of the emanation from these gases can be effected as follows.

The gases are transferred over mercury from the tube $E$ into the reservoir $R$ through the capillary tube $K$ (fig. 59). To remove

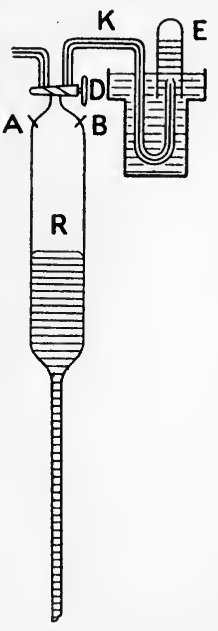

Fig. 59. the oxygen and hydrogen, the three-way tap $D$ is then closed and the pressure inside the reservoir diminished by lowering the mercury. A spark is then passed between the two electrodes $A$ and $B$ by means of an induction coil. The oxygen and hydrogen combine, and the volume of the gas is thereby diminished as a rule to about one-fifth of its original volume.

To further purify the emanation, the gases remaining are exposed for some hours to caustic potash, which removes carbon dioxide and water vapour. To carry out this process, a small glass tube is coated with a thin film of potash R.-A. 
obtained by placing a small piece of potash in the tube and melting it by gently warming. The tube is then filled with mercury and inverted in a trough containing mercury; the emanation is then introduced.

For further purification the emanation is condensed by passing it through a tube immersed in liquid air. When completely condensed the remaining gases are pumped off. The condensation can be effected by means of the apparatus shown in figure 60. The gases are introduced into the gas

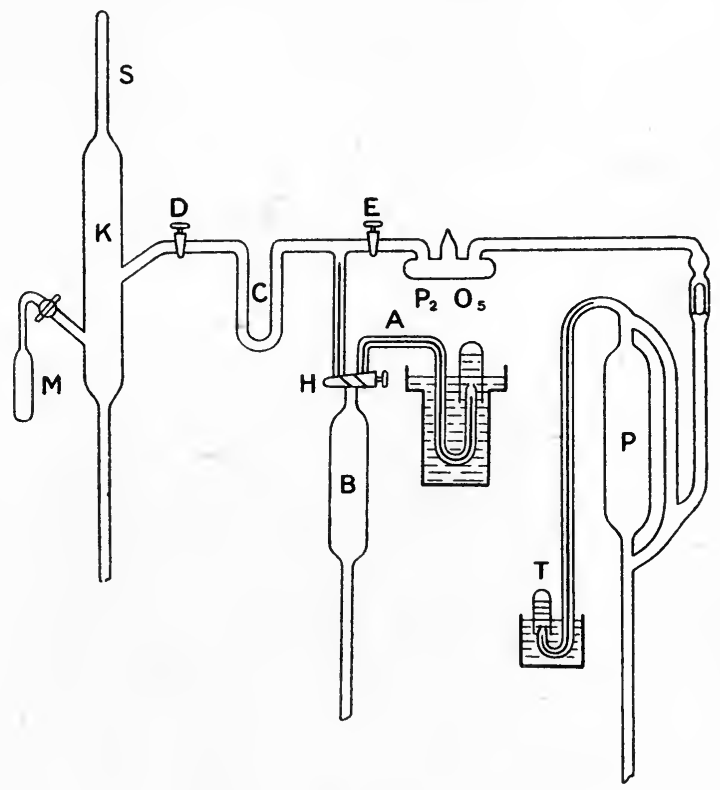

FIc. 60 .

holder $B$ through the capillary $A$, and then into the apparatus through the three way stop-cock $H$, the taps $D$ and $E$ being closed. The narrow U-tube $C$ is immersed in liquid air to condense the emanation. The gases are slowly forced into the $U$-tube by raising the mercury in $B$ and then sucked back again. After repeating this process several times the emanation will be nearly completely condensed, as indicated by the phosphoresence of the walls of the U-tube. The 
condensation is usually effected in the course of a few minutes. It is well first to condense the emanation near the bottom of the U-tube and then to raise the level of the liquid air. The uncondensed gases can be pumped off by lowering the level of the mercury in $B$; or the tap $H$ may be closed and the gases pumped off through the tap $E$ by the Toepler pump $P$. Since traces of emanation are invariably delivered into the pump, it is essential that the uncondensed gases should not be pumped out into the room, but collected in the tube $T$. The purified emanation can be introduced into any required apparatus through the reservoir $K$, previously completely evacuated by the Toepler pump and by the bulb $M$ containing charcoal immersed in liquid air. The taps $E$ and $H$ are closed and the liquid air surrounding the U-tube is removed. The tap $D$ is opened and the emanation allowed to pass into $K$. Since the volume of the reservoir $K$ is large compared with that of the U-tube, most of the emanation will at once pass into $K$. To transfer the last traces from the $U$-tube, the emanation is condensed by applying to the outside of the glass tube $K$ a plug of cotton wool soaked in liquid air. When all the emanation has been removed from the $U$-tube the tap $D$ is closed. By raising the mercury the emanation is finally transferred from $K$ into the tube $S$, which is then sealed off in a blow-pipe.

When perfectly pure one curie of emanation occupies 0.58 c.mm. The emanation finally obtained in the above purification will be found to be of about 30 to 50 per cent. purity, which suffices for most purposes. If perfectly pure emanation is required, extreme care has to be taken in all the manipulations. In particular, all stop-cocks must be lubricated with phosphorus pentoxide to avoid tap grease from which carbon dioxide is produced.

The tube $S$ is designed according to the requirements of the experiment in which it is to be used. To serve as a strong source of $a$ radiation, it may be made of thick-walled glass tubing drawn out so as to be conical in shape, as shown in figure 61. The end of the tube, which is ground flat, is closed by_attaching to it with sealing-wax a thin sheet of mica $M$. 
If the internal diameter of the tube is not more than three millimetres at the point of attachment of the mica, a sheet of mica equivalent in stopping power to as little as one centimetre of air will stand a pressure of one atmosphere, and the tube can therefore be exhausted without fear of breakage. In the construction of such a tube, care must, however, be taken to use as little sealing-wax as possible, for the emanation

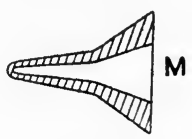

FIG. 61.

gradually decomposes it with evolution of gases, the production of which may ultimately raise the pressure inside the tube sufficiently to break the mica window.

The use of wax is obviated by employing " $\alpha$-ray tubes" made of glass, so thin that the $\alpha$ rays can pass through the walls without much reduction in range. The making of such tubes demands considerable skill. A thin glass tube is fixed by a stopper at one end inside a hard glass tube of about one centimetre diameter. The outer tube is then heated in a blowpipe to gradually soften the inner tube. By gently pulling, the inner tube can be drawn out so thin that its walls correspond in stopping power to about one centimetre of air. If the diameter of this tube does not exceed one millimetre it can be exhausted without fear of its collapsing. Such tubes were first used by Rutherford to identify the $\alpha$ particle with helium and were constructed by Baumbach.

When using large quantities of emanation $\alpha$-ray tubes should be handled with great caution, for they constitute a very powerful source of radiation, and if held in the hand too long may produce "burns" which are very difficult to heal. In all the manipulations involved in extracting the emanation and filling tubes with it, it is important to take precautions to prevent the escape of radium emanation as far as possible; for the emanation diffuses about and may contaminate electroscopes throughout the laboratory. It is 


\section{IX.] SEPARATION OF RADIO-ACTIVE SUBSTANCES 133}

advisable to set aside a special room, preferably at the top of the building, for the purpose of separating the emanation from the radium. In case of an accidental escape of emanation, the windows should at once be opened. It is advisable not to work with electroscopes immediately aftér transferring the emanation, for it is difficult to prevent the hands and clothes becoming slightly active in the process. 


\section{APPENDIX I.}

\section{TABLE OF RADIO-ACTIVE CONSTANTS.}

Charge carried by a hydrogen atom

$$
=4.65 \times 10^{-10} \text { E.S. unit }=1.55 \times 10^{-20} \text { E.M. unit. }
$$

Charge carried by an $\alpha$ particle

$$
=9 \cdot 3 \times 10^{-10} \text { E.S. unit }=3 \cdot 1 \times 10^{-20} \text { E.M. unit. }
$$

Mass of hydrogen atom $=1.61 \times 10^{-24}$ gram.

Number of atoms in one gram of hydrogen $=6 \cdot 2 \times 10^{23}$.

Number of molecules per c.c. of any gas at standard temperature and pressure $=2 \cdot 72 \times 10^{19}$.

Number of $\alpha$ particles expelled per second by one gram of radium (without its disintegration products) $=3.4 \times 10^{10}$.

Number of $a$ particles expelled per second by one gram of uranium $=2.37 \times 10^{4}$.

Number of $a$ particles expelled per second by one gram of thorium in equilibrium with its disintegration products $=2.7 \times 10^{4}$.

Volume of emanation in equilibrium with one gram of radium $=\cdot 585$ c.mm.

Production of helium per year per gram of radium in equilibrium with its disintegration products $=158 \mathrm{c} . \mathrm{mm}$.

Heating effect of one gram of radium (including disintegration products) $=132$ gram calories per hour.

Energy necessary to produce one pair of ions $=5 \cdot 2 \times 10^{-11} \mathrm{erg}$.

$\frac{e}{m}$ for slow $\beta$ rays $=5.595 \times 10^{17}$ E.s. units

$=1.865 \times 10^{7}$ E.M. units.

$\frac{e}{m}$ for $\alpha$ rays $\quad=1.52 \times 10^{14}$ E.s. units

$=5.07 \times 10^{3}$ E.M. units. 


\section{RANGES OF $\alpha$ PARTICLES.}

\begin{tabular}{|c|c|c|c|c|c|c|}
\hline \multirow{2}{*}{\multicolumn{3}{|c|}{ Substance. }} & \multicolumn{2}{|c|}{ Ranges in air at N.P. } & \multirow{3}{*}{$\begin{array}{c}\begin{array}{c}\text { Initial Velocity } \\
\text { in } \mathrm{cm} \text {. per sec. }\end{array} \\
1.47 \times 10^{9}\end{array}$} & \multirow{3}{*}{$\frac{\begin{array}{c}\text { Total Number of } \\
\text { Ions Produced. }\end{array}}{1.26 \times 10^{5}}$} \\
\hline & & & \multirow{2}{*}{$\frac{0^{\circ} \mathrm{C} .}{2 \cdot 37 \mathrm{~cm} .}$} & \multirow{2}{*}{$\frac{15^{\circ} \mathrm{C} .}{2.50 \mathrm{~cm} .}$} & & \\
\hline Uranium 1 & - & - & & & & \\
\hline Uranium 2 & - & - & $2 \cdot 75$ & $2 \cdot 90$ & $1 \cdot 54$ & $1 \cdot 37$ \\
\hline Ionium - & - & - & $2 \cdot 85$ & $3 \cdot 00$ & $1 \cdot 56$ & $1 \cdot 41$ \\
\hline Radium - & - & - & $3 \cdot 13$ & $3 \cdot 30$ & $1 \cdot 61$ & $1 \cdot 50$ \\
\hline Radium Em & nat & & $3 \cdot 94$ & $4 \cdot 16$ & $1 \cdot 74$ & $1 \cdot 73$ \\
\hline Radium $A$ & - & - & $4 \cdot 50$ & $4 \cdot 75$ & $1 \cdot 82$ & $1 \cdot 88$ \\
\hline Radium $C$ & - & - & $6 \cdot 57$ & $6 \cdot 94$ & $2 \cdot 06$ & $2 \cdot 37$ \\
\hline Radium $F$ & - & - & $3 \cdot 58$ & $3 \cdot 77$ & $1 \cdot 68$ & $1 \cdot 64$ \\
\hline Thorium & - & - & $2 \cdot 58$ & $2 \cdot 7 \cdot 2$ & $1 \cdot 51$ & $1 \cdot 32$ \\
\hline Radio-thoriv & & - & $3 \cdot 67$ & $3 \cdot 87$ & $1 \cdot 70$ & $1 \cdot 66$ \\
\hline Thorium $X$ & - & - & $4 \cdot 08$ & $4 \cdot 30$ & $1 \cdot 75$ & $1 \cdot 77$ \\
\hline Thorium Em & ana & & $4 \cdot 74$ & $5 \cdot 00$ & $1 \cdot 85$ & $1 \cdot 93$ \\
\hline Thorium $A$ & - & - & $5 \cdot 40$ & $5 \cdot 70$ & $1 \cdot 93$ & $2 \cdot 10$ \\
\hline Thorium $C_{1}$ & $\cdot$ & - & $4 \cdot 55$ & $4 \cdot 80$ & $1 \cdot 82$ & $1 \cdot 89$ \\
\hline Thorium $C_{2}$ & $\cdot$ & - & $8 \cdot 16$ & $8 \cdot 60$ & $2 \cdot 21$ & $2 \cdot 74$ \\
\hline Radio-actini & & - & $4 \cdot 36$ & $4 \cdot 60$ & $1 \cdot 80$ & $1 \cdot 84$ \\
\hline Actinium $X$ & - & - & $4 \cdot 17$ & $4 \cdot 40$ & $1 \cdot 77$ & $1 \cdot 79$ \\
\hline Actinium En & tana & on & $5 \cdot 40$ & $5 \cdot 70$ & 1.93 & $2 \cdot 10$ \\
\hline Actinium $A$ & - & - & $6 \cdot 16$ & $6 \cdot 50$ & $2 \cdot 02$ & $2 \cdot 27$ \\
\hline Actinium $C$ & - & $\cdot$ & $5 \cdot 12$ & $5 \cdot 40$ & $1 \cdot 89$ & $2 \cdot 02$ \\
\hline
\end{tabular}




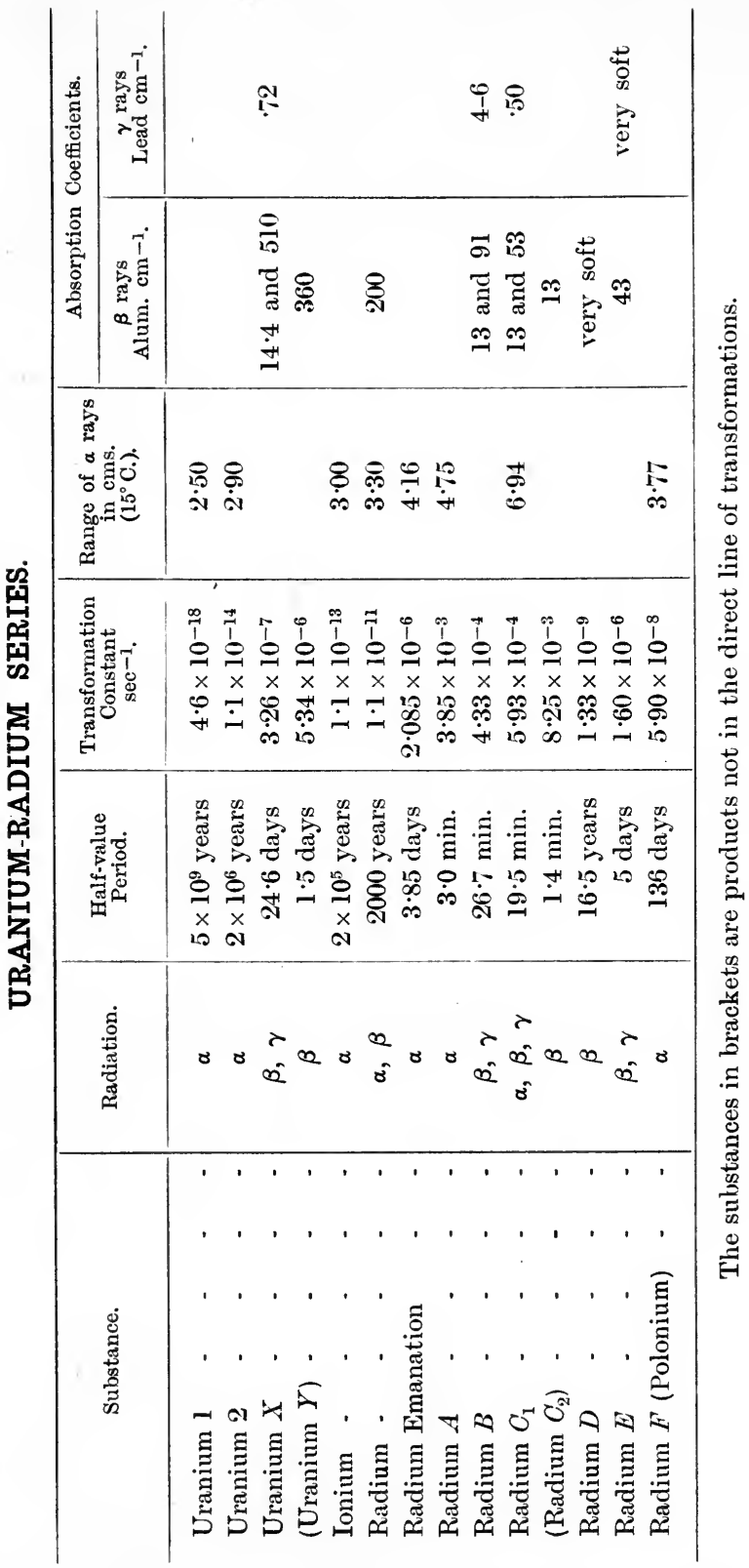


APPENDIX I.

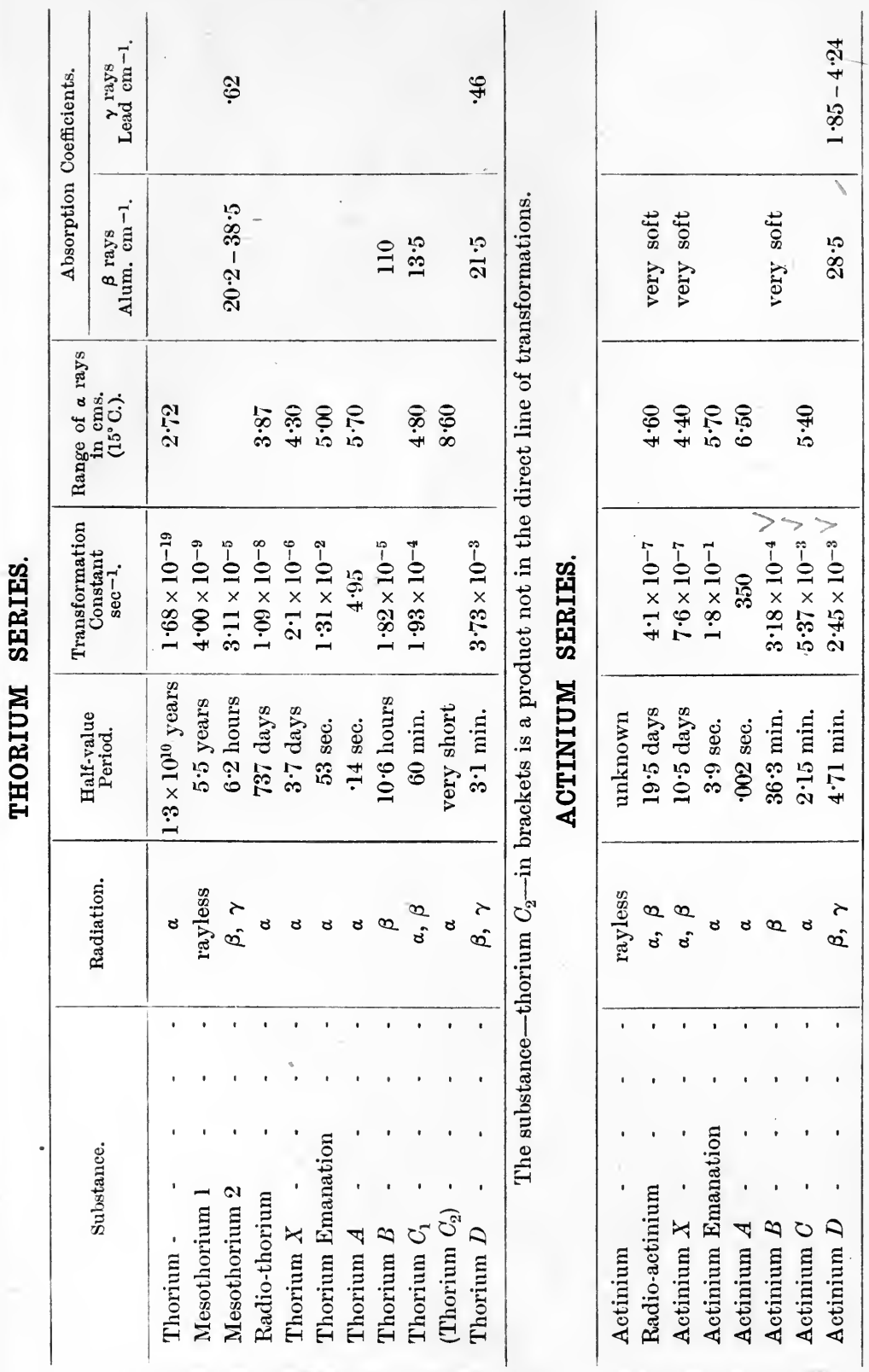


APPENDIX II.

\section{DECAY OF RADIUM EMANATION.}

$T_{1}=3.85$ days,$\quad \lambda_{1}=2.085 \times 10^{-6} \mathrm{sec}^{-1}$.

\begin{tabular}{|c|c|c|c|c|c|}
\hline Time. & Activity. & Time. & Activity. & Time. & Activity. \\
\hline & $100 \cdot 0$ & 4 days. & $48 \cdot 7$ & 8 days. & $23 \cdot 7$ \\
\hline 3 hrs. & $97 \cdot 8$ & 3 hrs. & $47 \cdot 6$ & $8 \cdot 5$ & $21 \cdot 7$ \\
\hline 6 & $95 \cdot 6$ & 6 & $46 \cdot 5$ & $9 \cdot 0$ & $19 \cdot 8$ \\
\hline 9 & $93 \cdot 5$ & 9 & $45 \cdot 5$ & $9 \cdot 5$ & $18 \cdot 1$ \\
\hline 12 & $91 \cdot 4$ & 12 & $44 \cdot 5$ & $10 \cdot 0$ & $16 \cdot 6$ \\
\hline 15 & $89 \cdot 4$ & 15 & $43 \cdot 5$ & $10 \cdot 5$ & $15 \cdot 1$ \\
\hline 18 & $87 \cdot 4$ & 18 & $42 \cdot 5$ & $11 \cdot 0$ & $13 \cdot 8$ \\
\hline 21 & $85 \cdot 4$ & 21 & $41 \cdot 6$ & $11 \cdot 5$ & $12 \cdot 6$ \\
\hline 1 day. & $83 \cdot 5$ & 5 days. & $40 \cdot 6$ & $12 \cdot 0$ & $11 \cdot 5$ \\
\hline 3 hrs. & $81 \cdot 7$ & 3 hrs. & $39 \cdot 7$ & $12 \cdot 5$ & $10 \cdot 5$ \\
\hline 6 & . $\quad 79.9$ & 6 & $38 \cdot 9$ & $13 \cdot 0$ & $9 \cdot 63$ \\
\hline 9 & $78 \cdot 1$ & 9 & $38 \cdot 0$ & $13 \cdot 5$ & $8 \cdot 80$ \\
\hline 12 & $76 \cdot 3$ & 12 & $37 \cdot 2$ & $14 \cdot 0$ & $8 \cdot 04$ \\
\hline 15 & $74 \cdot 6$ & 15 & $36 \cdot 3$ & 15 & $6 \cdot 72$ \\
\hline 18 & $73 \cdot 0$ & 18 & $35 \cdot 5$ & 16 & $5 \cdot 61$ \\
\hline 21 & $71 \cdot 4$ & 21 & $34 \cdot 7$ & 17 & $4 \cdot 68$ \\
\hline 2 days. & $69 \cdot 8$ & 6 days. & $33 \cdot 9$ & 18 & 3.91 \\
\hline 3 & $68 \cdot 2$ & 3 & $33 \cdot 2$ & 19 & $3 \cdot 27$ \\
\hline 6 & $66 \cdot 7$ & 6 & $32 \cdot 5$ & 20 & $2 \cdot 73$ \\
\hline 9 & $65 \cdot 2$ & 9 & $31 \cdot 7$ & 21 & $2 \cdot 28$ \\
\hline 12 & $63 \cdot 7$ & 12 & $31 \cdot 0$ & 22 & 1.90 \\
\hline 15 & $62 \cdot 3$ & 15 & $30 \cdot 3$ & 23 & $1 \cdot 59$ \\
\hline 18 & $61 \cdot 0$ & 18 & $29 \cdot 7$ & 24 & $1 \cdot 33$ \\
\hline 21 & $59 \cdot 6$ & 21 & $29 \cdot 0$ & 25 & $1 \cdot 11$ \\
\hline 3 days. & $58 \cdot 3$ & 7 days. & $28 \cdot 4$ & 26 & .927 \\
\hline $3 \mathrm{hrs}$. & $57 \cdot 0$ & $3 \mathrm{hrs}$. & $27 \cdot 7$ & 27 & .774 \\
\hline 6 & $55 \cdot 7$ & 6 & $27 \cdot 1$ & 28 & $\cdot 647$ \\
\hline 9 & $54 \cdot 5$ & 9 & $26 \cdot 5$ & 29 & .540 \\
\hline 12 & $53 \cdot 3$ & 12 & $25 \cdot 9$ & 30 & .451 \\
\hline 15 & $52 \cdot 1$ & 15 & $25 \cdot 3$ & & \\
\hline 18 & $50 \cdot 9$ & 18 & $24 \cdot 8$ & & \\
\hline 21 & $49 \cdot 8$ & 21 & $24 \cdot 2$ & & \\
\hline
\end{tabular}




\section{DECAY OF ACTIVE DEPOSIT OF RADIUM.}

For radium $A, \quad T_{2}=3$ minutes, $\quad \lambda_{2}=3.85 \times 10^{-3} \mathrm{sec}^{-1}$.

For radium $B, \quad T_{3}=26.7$ minutes, $\quad \lambda_{3}=4.33 \times 10^{-4} \mathrm{sec}^{-1}$.

For radium $C, \quad T_{4}=19.5$ minutes,$\quad \lambda_{4}=5.93 \times 10^{-4} \mathrm{sec}^{-1}$.

\begin{tabular}{|c|c|c|c|c|}
\hline $\begin{array}{c}\text { Time } \\
\text { in minutes. }\end{array}$ & $\begin{array}{l}\text { Radium } A \\
\text { alone. }\end{array}$ & $\begin{array}{l}\text { Radium } B \\
\text { alone. }\end{array}$ & $\begin{array}{l}\text { Radium } C \\
\text { alone. }\end{array}$ & $\begin{array}{c}\text { Activity } \\
\text { measured by } \\
\text { rays from Ra } C \\
\text { (long exposure } \\
\text { to emanation). }\end{array}$ \\
\hline 0 & 100 & 100 & 100 & 100 \\
\hline 1 & $79 \cdot 37$ & $97 \cdot 43$ & $96 \cdot 51$ & $99 \cdot 97$ \\
\hline 2 & -63.00 & $94 \cdot 93$ & $93 \cdot 13$. & $99 \cdot 95$ \\
\hline 3 & $50 \cdot 00$ & $92 \cdot 50$ & $89 \cdot 88$ & $99 \cdot 89$ \\
\hline 4 & $39 \cdot 69$ & $90 \cdot 12$ & $86 \cdot 74$ & $99 \cdot 78$ \\
\hline 5 & $31 \cdot 50$ & $87 \cdot 81$ & $83 \cdot 70$ & $99 \cdot 63$ \\
\hline 6 & $25 \cdot 00$ & $85 \cdot 56$ & $80 \cdot 78$ & $99 \cdot 42$ \\
\hline 7 & $19 \cdot 84$ & $83 \cdot 36$ & $77 \cdot 96$ & $99 \cdot 2$ \\
\hline 8 & $15 \cdot 75$ & $81 \cdot 22$ & $75 \cdot 23$ & $98 \cdot 8$ \\
\hline 9 & $12 \cdot 50$ & $79 \cdot 14$ & $72 \cdot 60$ & $98 \cdot 5$ \\
\hline 10 & $9 \cdot 92$ & $77 \cdot 11$ & $70 \cdot 06$ & $98 \cdot 1$ \\
\hline 11 & $7 \cdot 88$ & $75 \cdot 13$ & $67 \cdot 62$ & $97 \cdot 6$ \\
\hline 12 & $6 \cdot 25$ & $73 \cdot 20$ & $65 \cdot 25$ & $97 \cdot 0$ \\
\hline 13 & $4 \cdot 96$ & $71 \cdot 32$ & $62 \cdot 97$ & $96 \cdot 5$ \\
\hline 14 & $3 \cdot 94$ & $69 \cdot 49$ & $60 \cdot 77$ & $95 \cdot 8$ \\
\hline 15 & $3 \cdot 12$ & $67 \cdot 71$ & $58 \cdot 65$ & $95 \cdot 1$ \\
\hline 16 & $2 \cdot 48$ & $65 \cdot 97$ & $56 \cdot 60$ & $94 \cdot 4$ \\
\hline 17 & 1.97 & $64 \cdot 28$ & $54 \cdot 62$ & $93 \cdot 7$ \\
\hline 18 & $1 \cdot 56$ & $62 \cdot 63$ & $52 \cdot 71$ & $92 \cdot 9$ \\
\hline 19 & $1 \cdot 24$ & $61 \cdot 02$ & $50 \cdot 87$ & $92 \cdot 1$ \\
\hline 20 & 0.99 & $59 \cdot 46$ & $49 \cdot 09$ & $91 \cdot 2$ \\
\hline 21 & 0.78 & $57 \cdot 93$ & $47 \cdot 37$ & $90 \cdot 3$ \\
\hline 22 & 0.62 & $56 \cdot 44$ & $45 \cdot 72$ & $89 \cdot 4$ \\
\hline 23 & $0 \cdot 49$ & $55 \cdot 00$ & $44 \cdot 12$ & $88 \cdot 5$ \\
\hline 24 & $0 \cdot 39$ & $53 \cdot 58$ & $42 \cdot 58$ & $87 \cdot 5$ \\
\hline 25 & $0 \cdot 31$ & $52 \cdot 21$ & $41 \cdot 09$ & $86 \cdot 5$ \\
\hline 26 & $0 \cdot 25$ & $50 \cdot 87$ & $39 \cdot 65$ & $85 \cdot 5$ \\
\hline 27 & 0.20 & $49 \cdot 57$ & $38 \cdot 27$ & $84 \cdot 5$ \\
\hline 28 & $0 \cdot 16$ & $48 \cdot 29$ & $36 \cdot 93$ & $83 \cdot 5$ \\
\hline 29 & $0 \cdot 12$ & $47 \cdot 05$ & $35 \cdot 64$ & $82 \cdot 4$ \\
\hline 30 & $0 \cdot 10$ & $45 \cdot 85$ & $34 \cdot 39$ & $81 \cdot 3$ \\
\hline 31 & - & $44 \cdot 67$ & $33 \cdot 19$ & $80 \cdot 3$ \\
\hline 32 & - & $43 \cdot 52$ & 32.03 & $79 \cdot 3$ \\
\hline 33 & - & $42 \cdot 40$ & $30 \cdot 91$ & $78 \cdot 2$ \\
\hline 34 & - & $41 \cdot 32$ & $29 \cdot 83$ & $77 \cdot 1$ \\
\hline
\end{tabular}


APPENDIX II.

\begin{tabular}{|c|c|c|c|c|}
\hline $\begin{array}{c}\text { Time } \\
\text { in minutes. }\end{array}$ & $\begin{array}{c}\text { Radium } A \\
\text { alone. } \\
\text { ' }\end{array}$ & $\begin{array}{l}\text { Radium } B \\
\text { alone. }\end{array}$ & $\begin{array}{c}\text { Radium } C . \\
\text { alone. }\end{array}$ & $\begin{array}{c}\text { Activity } \\
\text { measured by } \\
\text { rays from Ra } C \\
\text { (long exposure } \\
\text { to emanation). }\end{array}$ \\
\hline 35 & - & $40 \cdot 26$ & $28 \cdot 79$ & $76 \cdot 0$ \\
\hline 36 & - & $39 \cdot 23$ & $27 \cdot 78$ & $74 \cdot 9$ \\
\hline 37 & - & $38 \cdot 22$ & $26 \cdot 81$ & $73 \cdot 8$ \\
\hline 38 & - & $37 \cdot 24$ & $25 \cdot 88$ & $72 \cdot 7$ \\
\hline 39 & - & $36 \cdot 28$ & $24 \cdot 97$ & $71 \cdot 6$ \\
\hline 40 & - & $35 \cdot 34$ & $24 \cdot 10$ & 70.5 \\
\hline 41 & - & $34 \cdot 44$ & $23 \cdot 26$ & $69 \cdot 4$ \\
\hline 42 & - & $33 \cdot 56$ & $22 \cdot 44$ & $68 \cdot 3$ \\
\hline 43 & - & $32 \cdot 70$ & $21 \cdot 66$ & $67 \cdot 3$ \\
\hline 44 & - & $31 \cdot 86$ & $20 \cdot 90$ & $66 \cdot 2$ \\
\hline 45 & - & $31 \cdot 04$ & $20 \cdot 17$ & $65 \cdot 1$ \\
\hline 46 & - & $30 \cdot 24$ & $19 \cdot 47$ & $64 \cdot 0$ \\
\hline 47 & - & $29 \cdot 47$ & $18 \cdot 79$ & $63 \cdot 0$ \\
\hline 48 & - & $28 \cdot 71$ & $18 \cdot 13$ & $62 \cdot 0$ \\
\hline 49 & - & 27.98 & $17 \cdot 50$ & 60.9 \\
\hline 50 & - & $27 \cdot 26$ & $16 \cdot 88$ & $59 \cdot 8$ \\
\hline 51 & - & $26 \cdot 56$ & $16 \cdot 29$ & $58 \cdot 8$ \\
\hline 52 & - & $25 \cdot 88$ & $15 \cdot 73$ & $57 \cdot 8$ \\
\hline 53 & - & $25 \cdot 21$ & $15 \cdot 18$ & $56 \cdot 8$ \\
\hline 54 & - & $24 \cdot 57$ & $14 \cdot 65$ & $55 \cdot 8$ \\
\hline 55 & - & $23 \cdot 94$ & $14 \cdot 13$ & $54 \cdot 8$ \\
\hline 56 & - & $23 \cdot 32$ & $13 \cdot 64$ & $53 \cdot 8$ \\
\hline 57 & - & $22 \cdot 72$ & $13 \cdot 16$ & $52 \cdot 8$ \\
\hline 58 & - & $22 \cdot 14$ & $12 \cdot 70$ & $51 \cdot 8$ \\
\hline 59 & - & $21 \cdot 57$ & $12 \cdot 26$ & 50.9 \\
\hline 60 & - & $21 \cdot 02$ & $11 \cdot 83$ & $49 \cdot 9$ \\
\hline 61 & - & $20 \cdot 48$ & $11 \cdot 42$ & $48 \cdot 9$ \\
\hline 62 & - & $19 \cdot 95$ & $11 \cdot 02$ & $48 \cdot 0$ \\
\hline 63 & - & $19 \cdot 44$ & $10 \cdot 63$ & $47 \cdot 1$ \\
\hline 64 & - & 18.94 & $10 \cdot 26$ & $46 \cdot 3$ \\
\hline 65 & - & $18 \cdot 46$ & $9 \cdot 90$ & $45 \cdot 4$ \\
\hline 66 & - & $17 \cdot 98$ & $9 \cdot 56$ & $44 \cdot 5$ \\
\hline 67 & - & $17 \cdot 52$ & $9 \cdot 22$ & $43 \cdot 7$ \\
\hline 68 & - & $17 \cdot 07$ & $8 \cdot 90$ & $42 \cdot 8$ \\
\hline 69 & - & $16 \cdot 63$ & $8 \cdot 59$ & $42 \cdot 0$ \\
\hline 70 & - & $16 \cdot 21$ & $8 \cdot 29$ & $41 \cdot 1$ \\
\hline 71 & - & $15 \cdot 79$ & $8 \cdot 00$ & $40 \cdot 3$ \\
\hline 72 & - & $15 \cdot 39$ & $7 \cdot 72$ & $39 \cdot 5$ \\
\hline 73 & - & 14.99 & $7 \cdot 45$ & $38 \cdot 7$ \\
\hline 74 & - & $14 \cdot 61$ & $7 \cdot 19$ & $38 \cdot 0$ \\
\hline
\end{tabular}




\begin{tabular}{|c|c|c|c|c|}
\hline $\begin{array}{c}\text { Time } \\
\text { in minutes. }\end{array}$ & $\begin{array}{l}\text { Radium } A \\
\text { alone. }\end{array}$ & $\begin{array}{c}\text { Radium } B \\
\text { alone. }\end{array}$ & $\begin{array}{l}\text { Radium } C \\
\text { alone. }\end{array}$ & $\begin{array}{l}\text { Activity } \\
\text { measured by } \\
\text { rays from Ra } C \\
\text { (long exposure } \\
\text { to emanation). }\end{array}$ \\
\hline 75 & - & $14 \cdot 23$ & $6 \cdot 94$ & $37 \cdot 3$ \\
\hline 76 & - & $13 \cdot 87$ & $6 \cdot 69$ & $36 \cdot 5$ \\
\hline 77 & - & 13.51 & $6 \cdot 46$ & $35 \cdot 8$ \\
\hline 78 & - & $13 \cdot 16$ & $6 \cdot 24$ & $35 \cdot 0$ \\
\hline 79 & - & $12 \cdot 83$ & $6 \cdot 02$ & $34 \cdot 3$ \\
\hline 80 & - & $12 \cdot 50$ & $5 \cdot 81$ & $33 \cdot 6$ \\
\hline 81 & - & $12 \cdot 18$ & $5 \cdot 60$ & $32 \cdot 9$ \\
\hline 82 & - & $11 \cdot 86$ & $5 \cdot 41$ & $32 \cdot 2$ \\
\hline 83 & - & $11 \cdot 56$ & $5 \cdot 22$ & $31 \cdot 6$ \\
\hline 84 & - & $11 \cdot 26$ & 5.04 & 30.9 \\
\hline 85 & - & 10.97 & $4 \cdot 86$ & $30 \cdot 3$ \\
\hline 86 & - & $10 \cdot 69$ & $4 \cdot 69$ & $29 \cdot 7$ \\
\hline 87 & - & $10 \cdot 42$ & $4 \cdot 53$ & $29 \cdot 0$ \\
\hline 88 & - & $10 \cdot 15$ & $4 \cdot 37$ & $28 \cdot 4$ \\
\hline 89 & - & $9 \cdot 89$ & $4 \cdot 22$ & $27 \cdot 8$ \\
\hline 90 & - & $9 \cdot 64$ & $4 \cdot 07$ & $27 \cdot 2$ \\
\hline 91 & - & $9 \cdot 39$ & $3 \cdot 93$ & $26 \cdot 6$ \\
\hline 92 & - & $9 \cdot 15$ & $3 \cdot 79$ & $26 \cdot 1$ \\
\hline 93 & - & 8.91 & $3 \cdot 66$ & $25 \cdot 6$ \\
\hline 94 & - & $8 \cdot 68$ & 3.53 & $25 \cdot 0$ \\
\hline 95 & - & $8 \cdot 46$ & $3 \cdot 41$ & $24 \cdot 4$ \\
\hline 96 & - & $8 \cdot 24$ & $3 \cdot 29$ & $23 \cdot 9$ \\
\hline 97 & - & $8 \cdot 03$ & $3 \cdot 17$ & $23 \cdot 4$ \\
\hline 98 & - & $7 \cdot 83$ & 3.06 & $22 \cdot 9$ \\
\hline 99 & - & $7 \cdot 63$ & 2.95 & $22 \cdot 4$ \\
\hline 100 & - & $7 \cdot 43$ & $2 \cdot 85$ & $21 \cdot 9$ \\
\hline 101 & - & $7 \cdot 24$ & $2 \cdot 75$ & $21 \cdot 4$ \\
\hline 102 & - & $7 \cdot 05$ & $2 \cdot 66$ & $21 \cdot 0$ \\
\hline 103 & - & $6 \cdot 87$ & 2.55 & 20.5 \\
\hline 104 & - & $6 \cdot 69$ & $2 \cdot 47$ & $20 \cdot 1$ \\
\hline 105 & - & 6.52 & $2 \cdot 39$ & $19 \cdot 6$ \\
\hline 106 & - & $6 \cdot 35$ & $2 \cdot 30$ & $19 \cdot 2$ \\
\hline 107 & - & $6 \cdot 19$ & $2 \cdot 22$ & $18 \cdot 8$ \\
\hline 108 & - & $6 \cdot 03$ & $2 \cdot 14$ & $18 \cdot 4$ \\
\hline 109 & - & $5 \cdot 88$ & $2 \cdot 07$ & $17 \cdot 9$ \\
\hline 110 & - & $5 \cdot 73$ & $2 \cdot 00$ & $17 \cdot 5$ \\
\hline 111 & - & $5 \cdot 58$ & 1.93 & $17 \cdot 1$ \\
\hline 112 & - & $5 \cdot 44$ & $1 \cdot 86$ & $16 \cdot 7$ \\
\hline 113 & - & $\mathbf{5} \cdot 30$ & 179 & $16 \cdot 4$ \\
\hline 114 & - & $5 \cdot 16$ & $1 \cdot 73$ & $16 \cdot 0$ \\
\hline
\end{tabular}




\begin{tabular}{|c|c|c|c|c|}
\hline $\begin{array}{c}\text { Time } \\
\text { in minutes. }\end{array}$ & $\begin{array}{l}\text { Radium } A \\
\text { alone. }\end{array}$ & $\begin{array}{l}\text { Radium } B \\
\text { alone. }\end{array}$ & $\begin{array}{c}\text { Radium } C \\
\text { alone. }\end{array}$ & $\begin{array}{c}\text { Activity } \\
\text { measured by } \\
\text { rays from Ra } C \\
\text { (long exposure } \\
\text { to emanation). }\end{array}$ \\
\hline 115 & - & $5 \cdot 03$ & $1 \cdot 67$ & $15 \cdot 7$ \\
\hline 116 & - & $4 \cdot 90$ & $1 \cdot 61$ & $15 \cdot 3$ \\
\hline 117 & - & $4 \cdot 77$ & 1.55 & $14 \cdot 9$ \\
\hline 118 & - & $4 \cdot 65$ & $1 \cdot 50$ & $14 \cdot 6$ \\
\hline 119 & - & $4 \cdot 53$ & $l \cdot 45$ & $14 \cdot 3$ \\
\hline 120 & - & $4 \cdot 42$ & $1 \cdot 40$ & $14 \cdot 0$ \\
\hline 121 & - & $4 \cdot 30$ & $1 \cdot 35$ & $13 \cdot 6$ \\
\hline 122 & - & $4 \cdot 20$ & $1 \cdot 30$ & $13 \cdot 4$ \\
\hline 123 & - & $4 \cdot 09$ & $1 \cdot 26$ & $13 \cdot 1$ \\
\hline 124 & - & $3 \cdot 98$ & $1 \cdot 22$ & $12 \cdot 7$ \\
\hline 125 & - & $3 \cdot 88$ & $1 \cdot 17$ & $12 \cdot 4$ \\
\hline 126 & - & $3 \cdot 78$ & $1 \cdot 13$ & $12 \cdot 1$ \\
\hline 127 & - & $3 \cdot 68$ & $1 \cdot 09$ & 11.9 \\
\hline 128 & - & $3 \cdot 59$ & $1 \cdot 05$ & $11 \cdot 6$ \\
\hline 129 & - & $3 \cdot 50$ & 1.02 & $11 \cdot 4$ \\
\hline 130 & - & $3 \cdot 41$ & .98 & $11 \cdot 1$ \\
\hline 131 & - & $3 \cdot 32$ & .95 & $10 \cdot 9$ \\
\hline 132 & - & $3 \cdot 23$ & .92 & $10 \cdot 6$ \\
\hline 133 & - & $3 \cdot 15$ & $\cdot 88$ & $10 \cdot 3$ \\
\hline 134 & - & $3 \cdot 07$ & $\cdot 85$ & $10 \cdot 1$ \\
\hline 135 & - & $2 \cdot 99$ & $\cdot 82$ & $9 \cdot 9$ \\
\hline 136 & - & $2 \cdot 91$ & .79 & $9 \cdot 6$ \\
\hline 137 & - & $2 \cdot 84$ & .76 & $9 \cdot 3$ \\
\hline 138 & - & $2 \cdot 77$ & .74 & $9 \cdot 2$ \\
\hline 139 & - & $2 \cdot 70$ & .71 & $9 \cdot 0$ \\
\hline 140 & - & $2 \cdot 63$ & .69 & $8 \cdot 8$ \\
\hline 141 & - & $2 \cdot 56$ & .66 & $8 \cdot 6$ \\
\hline 142 & - & $2 \cdot 49$ & .64 & $8 \cdot 4$ \\
\hline 143 & - & $2 \cdot 43$ & $\cdot 62$ & $8 \cdot 2$ \\
\hline 144 & - & $2 \cdot 37$ & $\cdot 60$ & $8 \cdot 0$ \\
\hline 145 & - & $2 \cdot 31$ & $\cdot 57$ & $7 \cdot 8$ \\
\hline 146 & - & $2 \cdot 25$ & 55 & $7 \cdot 6$ \\
\hline 147 & - & $2 \cdot 19$ & $\cdot 53$ & $7 \cdot 4$ \\
\hline 148 & - & $2 \cdot 13$ & $\cdot 52$ & $7 \cdot 2$ \\
\hline 149 & - & $2 \cdot 08$ & $\cdot 50$ & $7 \cdot 1$ \\
\hline 150 & - & $2 \cdot 02$ & $\cdot 48$ & $6 \cdot 9$ \\
\hline 151 & - & 1.97 & .46 & $6 \cdot 7$ \\
\hline 152 & - & 1.92 & .45 & $6 \cdot 6$ \\
\hline 153 & - & $1 \cdot 87$ & .43 & $6 \cdot 4$ \\
\hline 154 & - & 1.82 & .42 & $6 \cdot 3$ \\
\hline
\end{tabular}




\begin{tabular}{|c|c|c|c|c|}
\hline $\begin{array}{c}\text { Time } \\
\text { in minutes. }\end{array}$ & $\begin{array}{c}\text { Radium } A \\
\text { alone. }\end{array}$ & $\begin{array}{c}\text { Radium } B \\
\text { alone. }\end{array}$ & $\begin{array}{l}\text { Radium } C \\
\text { alone. }\end{array}$ & $\begin{array}{c}\text { Activity } \\
\text { measured by } \\
\text { rays from Ra } C \\
\text { (long exposure } \\
\text { to emanation). }\end{array}$ \\
\hline 155 & - & $1 \cdot 78$ & $\cdot 40$ & $6 \cdot 1$ \\
\hline 156 & -- & $1 \cdot 73$ & $\cdot 39$ & $6 \cdot 0$ \\
\hline 157 & - & $1 \cdot 69$ & $\cdot 38$ & $5 \cdot 9$ \\
\hline 158 & - & 1.64 & .36 & $5 \cdot 7$ \\
\hline 159 & - & $1 \cdot 60$ & $\cdot 35$ & $5 \cdot 6$ \\
\hline 160 & - & 1.56 & $\cdot 34$ & $5 \cdot 4$ \\
\hline 161 & - & 1.52 & $\cdot 33$ & $5 \cdot 3$ \\
\hline 162 & - & 1.48 & $\cdot 32$ & $5 \cdot 2$ \\
\hline 163 & - & 1.44 & $\cdot 31$ & $5 \cdot 1$ \\
\hline 164 & - & 1.41 & $\cdot 29$ & $4 \cdot 9$ \\
\hline 165 & - & $1 \cdot 37$ & .28 & $4 \cdot 8$ \\
\hline 166 & - & $1 \cdot 34$ & $\cdot 27$ & $4 \cdot 7$ \\
\hline 167 & - & $1 \cdot 30$ & $\cdot 26$ & $4 \cdot 6$ \\
\hline 168 & - & $1 \cdot 27$ & .25 & $4 \cdot 5$ \\
\hline 169 & - & $1 \cdot 23$ & $\cdot 24$ & $4 \cdot 4$ \\
\hline 170 & - & $1 \cdot 20$ & $\cdot 24$ & $4 \cdot 3$ \\
\hline 171 & - & $1 \cdot 17$ & $\cdot 23$ & $4 \cdot 2$ \\
\hline 172 & - & $1 \cdot 14$ & $\cdot 22$ & $4 \cdot 1$ \\
\hline 173 & - & $1 \cdot 11$ & .21 & $4 \cdot 0$ \\
\hline 174 & - & 1.09 & $\cdot 20$ & $3 \cdot 9$ \\
\hline 175 & - & 1.06 & $\cdot 20$ & $3 \cdot 8$ \\
\hline 176 & - & 1.03 & $\cdot 19$ & $3 \cdot 7$ \\
\hline 177 & - & 1.00 & $\cdot 19$ & $3 \cdot 6$ \\
\hline 178 & - & .98 & $\cdot 18$ & $3 \cdot 5$ \\
\hline 179 & - & .95 & $\cdot 17$ & $3 \cdot 4$ \\
\hline 180 & - & .93 & $\cdot 16$ & $3 \cdot 3$ \\
\hline
\end{tabular}

The following data will be of use for calculating decay and recovery curves. The constants $\lambda_{1}, \lambda_{2}, \lambda_{3}, \lambda_{4}$ refer to the emanation, radium $A$, radium $B$ and radium $C$ respectively.

$$
\begin{aligned}
\log \lambda_{1} & =\overline{6} \cdot 3191061, & \log \lambda_{2} & =\overline{3} \cdot 5854607, \\
\log \lambda_{3} & =\overline{4} \cdot 6364879, & \log \lambda_{4} & =\overline{4} \cdot 7730547, \\
\log \left(\lambda_{2}-\lambda_{1}\right) & =\overline{3} \cdot 5852255, & \log \left(\lambda_{3}-\lambda_{1}\right) & =\overline{4} \cdot 6343916, \\
\log \left(\lambda_{4}-\lambda_{1}\right) & =\overline{4} \cdot 7715250, & \log \left(\lambda_{2}-\lambda_{3}\right) & =\overline{3} \cdot 5336450, \\
\log \left(\lambda_{2}-\lambda_{4}\right) & =\overline{3} \cdot 5128178, & \log \left(\lambda_{4}-\lambda_{3}\right) & =\overline{4} \cdot 2041200 .
\end{aligned}
$$




\section{RISE AND FALL OF ACTIVITY FROM PURE RADIUM EMANATION UNDER DIFFERENT CONDITIONS.}

For emanation, $T_{1}=3.85$ days, $\quad \lambda_{1}=2.08 \times 10^{-6} \mathrm{sec}^{-1}$.

For radium $A, \quad T_{2}=3$ minutes, $\quad \lambda_{2}=3.85 \times 10^{-3} \mathrm{sec}^{-1}$.

For radium $B, \quad T_{3}=26.7$ minutes, $\quad \lambda_{3}=4.33 \times 10^{-4} \mathrm{sec}^{-1}$.

For radium $C, \quad T_{4}=19.5$ minutes, $\quad \lambda_{4}=5.93 \times 10^{-4} \mathrm{sec}^{-1}$.

\begin{tabular}{|c|c|c|c|c|}
\hline Minutes. & $\begin{array}{c}\text { II. } \\
\text { Calculated rise } \\
\text { of Radium } C \\
\text { from pure } \\
\text { emanation. }\end{array}$ & \begin{tabular}{c|} 
III. \\
Calculated rise \\
of ionisation by \\
$\gamma$ rays measured \\
through $3 \mathrm{mms}$. \\
of lead.
\end{tabular} & $\begin{array}{c}\text { IV. } \\
\text { Calculated fall } \\
\text { of Radium } C \\
\text { after removal } \\
\text { from } \\
\text { emanation. }\end{array}$ & $\begin{array}{c}\text { V. } \\
\text { Culculated fall } \\
\text { of ionisation by } \\
\gamma \text { rays measured } \\
\text { through } 3 \text { mms. } \\
\text { of lead. }\end{array}$ \\
\hline 0 & 0.0000 & 0.000 & $1 \cdot 0000$ & 1.000 \\
\hline 2 & $\cdot 000252$ & .00143 & .9995 & .998 \\
\hline 3 & .000801 & $\cdot 00323$ & .9989 & .997 \\
\hline 4 & .001770 & .00570 & .9978 & .994 \\
\hline 5 & $\cdot 003239$ & $\cdot 00885$ & .9963 & .991 \\
\hline 6 & .005244 & .0126 & .9942 & .987 \\
\hline 8 & .01097 & .0221 & .9884 & .978 \\
\hline 10 & $\cdot 01899$ & $\cdot 0338$ & .9805 & .966 \\
\hline 12 & .02925 & $\cdot 04752$ & .9703 & .952 \\
\hline 14 & .04154 & .06299 & .9582 & .938 \\
\hline 17 & .06343 & $\cdot 08897$ & .9368 & .912 \\
\hline 20 & .08876 & $\cdot 118$ & .9120 & .884 \\
\hline 25 & $\cdot 1368$ & $\cdot 170$ & .8650 & $\cdot 833$ \\
\hline 30 & $\cdot 1897$ & .225 & .8134 & $\cdot 779$ \\
\hline 40 & $\cdot 3015$ & $\cdot 338$ & $\cdot 7046$ & $\cdot 669$ \\
\hline 50 & .4118 & .446 & .5985 & 566 \\
\hline 60 & .5125 & .543 & .4987 & $\cdot 469$ \\
\hline 70 & $\cdot 6021$ & .629 & .4112 & $\cdot 385$ \\
\hline 90 & $\cdot 7440$ & $\cdot 763$ & $\cdot 2719$ & $\cdot 253$ \\
\hline 120 & $\cdot 8775$ & .888 & $\cdot 1396$ & $\cdot 129$ \\
\hline 150 & .9468 & .952 & .06913 & .0639 \\
\hline 180 & .9800 & .982 & .03347 & .0308 \\
\hline 240 & .9994 & 1.00 & .00752 & .00691 \\
\hline 258 & $1 \cdot 000$ & $1 \cdot 00$ & .00477 & $\cdot 00438$ \\
\hline 270 & & & .00352 & \\
\hline
\end{tabular}


Column II. gives the number of atoms of radium $C$ present at different times in a closed vessel after admitting pure emanation. The maximum value, which is taken as unity, occurs after 258 minutes. The numbers are calculated from the equation :

$$
N=\frac{\lambda_{2} \lambda_{3} \lambda_{4}}{.97213} \sum_{\lambda=1,2,3,4} \frac{e^{-\lambda_{1} t}}{\left(\lambda_{2}-\lambda_{1}\right)\left(\lambda_{3}-\lambda_{1}\right)\left(\lambda_{4}-\lambda_{1}\right)} .
$$

This equation represents the variation of activity measured through $2 \cdot 3 \mathrm{~cm}$. of lead.

Column III. gives the ionisation at different times after the introduction of pre emanat. in on the assumption that when in radio-active equilibriur, radium $B$ contributes 11.5 per cent. of the ionisation and radium $C^{4} 88^{\circ} 5$ per cent. The numbers are calculated from the equation

$$
\begin{aligned}
N_{\mathrm{III}}=\frac{\cdot 115}{.97213} & \lambda_{2} \lambda_{3} \sum_{\lambda=1,2,3} \frac{e^{-\lambda_{1} t}}{\left(\lambda_{2}-\lambda_{1}\right)\left(\lambda_{3}-\lambda_{1}\right)} \\
& +\frac{885}{.97213} \lambda_{2} \lambda_{3} \lambda_{4} \sum_{\lambda=1,2,3,4} \frac{e^{-\lambda_{1} t}}{\left(\lambda_{2}-\lambda_{1}\right)\left(\lambda_{3}-\lambda_{1}\right)\left(\lambda_{4}-\lambda_{1}\right)} .
\end{aligned}
$$

This equation gives the activity measured through $3 \mathrm{mms}$. of lead.

Column IV. gives the number of atoms of radium $C$ left in a vessel at different times after completely removing the emanation which has been confined in it for a long time. The numbers are calculated from the equation

$$
N_{\mathrm{lv}}=e^{-\lambda_{1} t}-\frac{\lambda_{2} \lambda_{3} \lambda_{4}}{1 \cdot 00891} \sum_{\lambda=1,2,3,4} \frac{e^{-\lambda_{1} t}}{\left(\lambda_{2}-\lambda_{1}\right)\left(\lambda_{3}-\lambda_{1}\right)\left(\lambda_{4}-\lambda_{1}\right)} .
$$

This equation gives the fall of activity measured through $2 \cdot 3 \mathrm{cms}$. of lead.

Column V. gives the fall of ionisation, reckoned on the same assumption as used in calculating column III., after completely removing from a vessel emanation which had been confined in it for a long time.

$$
\begin{aligned}
N_{\mathrm{v}}=e^{-\lambda_{1} t} & -\frac{\cdot 115}{1 \cdot 0085} \lambda_{2} \lambda_{3} \sum_{\lambda=1,2,3} \frac{e^{-\lambda_{1} t}}{\left(\lambda_{2}-\lambda_{1}\right)\left(\lambda_{3}-\lambda_{1}\right)} \\
& -\frac{.885}{1.0085} \lambda_{2} \lambda_{3} \lambda_{4} \sum_{\lambda=1,2,3,4} \frac{e^{-\lambda_{1} t}}{\left(\lambda_{2}-\lambda_{1}\right)\left(\lambda_{3}-\lambda_{1}\right)\left(\lambda_{4}-\lambda_{1}\right)} .
\end{aligned}
$$

This gives the rate of decay of radium active deposit, completely separated from emanation, measured through $3 \mathrm{mms}$. of lead. 
DECAY OF RADIUM F (POLONIUM).

$$
T=136 \text { days }, \quad \lambda=005095(\text { day })^{-1} .
$$

\begin{tabular}{l|c||c|c}
\hline Time. & $100 e^{-\lambda t}$. & Time. & $100 e^{-\lambda t}$. \\
\hline 0 & 100 & 7 weeks. & $77 \cdot 9$ \\
1 day. & $99 \cdot 49$ & 8 & $75 \cdot 2$ \\
2 & $98 \cdot 99$ & 9 & $72 \cdot 5$ \\
3 & $98 \cdot 48$ & 10 & $70 \cdot 0$ \\
4 & $97 \cdot 99$ & 11 & 67.5 \\
5 & $97 \cdot 48$ & 12 & $65 \cdot 2$ \\
6 & $96 \cdot 99$ & 13 & $62 \cdot 9$ \\
1 week. & $96 \cdot 49$ & 26 & $39 \cdot 4$ \\
2 & $93 \cdot 1$ & 39 & $24 \cdot 9$ \\
3 & $89 \cdot 8$ & 1 year. & $15 \cdot 6$ \\
4 & $86 \cdot 7$ & 2 & $2 \cdot 4$ \\
5 & $83 \cdot 7$ & 3 & $\cdot 38$ \\
6 & $80 \cdot 8$ & & \\
\hline
\end{tabular}


DECAY OF ACTINIUM ACTIVE DEPOSIT MEASURED BY RAYS FROM ACTINIUM C (LONG EXPOSURE).

For actinium $B, \quad T_{1}=36.3 \mathrm{~min} ., \quad \lambda_{1}=0191(\mathrm{~min} .)^{-1}$.

For actinium $C, \quad T_{2}=2 \cdot 15 \mathrm{~min}$., $\quad \lambda_{2}=\cdot 322(\min .)^{-1}$.

\begin{tabular}{|c|c|c|c|c|c|}
\hline $\begin{array}{l}\text { Time in } \\
\text { minutes. }\end{array}$ & Aetivity. & $\begin{array}{l}\text { Time in } \\
\text { minutes. }\end{array}$ & Activity. & $\begin{array}{l}\text { Time in } \\
\text { minutes. }\end{array}$ & Activity. \\
\hline 0 & 100 & 47 & $43 \cdot 3$ & 94 & $17 \cdot 7$ \\
\hline 1 & $99 \cdot 7$ & 48 & $42 \cdot 5$ & 95 & $17 \cdot 3$ \\
\hline 2 & $99 \cdot 0$ & 49 & $41 \cdot 7$ & 96 & $17 \cdot 0$ \\
\hline 3 & 98.0 & 50 & 40.9 & 97 & $16 \cdot 7$ \\
\hline 4 & $96 \cdot 7$ & 51 & $40 \cdot 1$ & 98 & $16 \cdot 4$ \\
\hline 5 & $95 \cdot 4$ & 52 & $39 \cdot 4$ & 99 & $16 \cdot 0$ \\
\hline 6 & $93 \cdot 9$ & 53 & $38 \cdot 6$ & 100 & $15 \cdot 7$ \\
\hline 7 & $92 \cdot 3$ & 54 & $37 \cdot 9$ & 101 & $15 \cdot 4$ \\
\hline 8 & $90 \cdot 8$ & 55 & $37 \cdot 2$ & 102 & $15 \cdot 2$ \\
\hline 9 & $89 \cdot 2$ & 56 & $36 \cdot 5$ & 103 & $14 \cdot 9$ \\
\hline 10 & $87 \cdot 6$ & 57 & $35 \cdot 8$ & 104 & $14 \cdot 6$ \\
\hline 11 & 86.0 & 58 & $35 \cdot 1$ & 105 & $14 \cdot 3$ \\
\hline 12 & $84 \cdot 4$ & 59 & $34 \cdot 4$ & 106 & $14 \cdot 0$ \\
\hline 13 & $82 \cdot 8$ & 60 & $33 \cdot 8$ & 107 & $13 \cdot 8$ \\
\hline 14 & $81 \cdot 3$ & 61 & $33 \cdot 1$ & 108 & $13 \cdot 5$ \\
\hline 15 & $79 \cdot 8$ & 62 & 32.5 & 109 & $13 \cdot 3$ \\
\hline 16 & $78 \cdot 3$ & 63 & $31 \cdot 9$ & 110 & $13 \cdot 0$ \\
\hline 17 & $76 \cdot 8$ & 64 & $31 \cdot 3$ & 111 & 12.8 \\
\hline 18 & $75 \cdot 4$ & 65 & $30 \cdot 7$ & 112 & $12 \cdot 5$ \\
\hline 19 & $73 \cdot 9$ & 66 & $30 \cdot 1$ & 113 & $12 \cdot 3$ \\
\hline 20 & $72 \cdot 5$ & 67 & $29 \cdot 6$ & 114 & $12 \cdot 0$ \\
\hline 21 & $71 \cdot 2$ & 68 & $29 \cdot 0$ & 115 & $11 \cdot 8$ \\
\hline 22 & $69 \cdot \overline{8}$ & 69 & $28 \cdot 5$ & 116 & $11 \cdot 6$ \\
\hline 23 & $68 \cdot 5$ & 70 & $27 \cdot 9$ & 117 & $11 \cdot 4$ \\
\hline 24 & $67 \cdot 2$ & 71 & $27 \cdot 4$ & 118 & $11 \cdot 2$ \\
\hline 25 & 65.9 & 72 & 26.9 & 119 & $10 \cdot 9$ \\
\hline 26 & $64 \cdot 7$ & 73 & $26 \cdot 4$ & 120 & $10 \cdot 7$ \\
\hline 27 & $63 \cdot 5$ & 74 & $25 \cdot 9$ & 125 & $9 \cdot 74$ \\
\hline 28 & $62 \cdot 3$ & 75 & $25 \cdot 4$ & 130 & 8.85 \\
\hline 29 & $61 \cdot 1$ & 76 & $24 \cdot 9$ & 135 & 8.05 \\
\hline 30 & 59.9 & 77 & $24 \cdot 4$ & 140 & $7 \cdot 31$ \\
\hline 31 & $58 \cdot 8$ & 78 & $24 \cdot 0$ & 145 & $6 \cdot 65$ \\
\hline 32 & $57 \cdot 7$ & 79 & 23.5 & 150 & 6.04 \\
\hline 33 & $56 \cdot 6$ & 80 & $23 \cdot 1$ & 155 & $5 \cdot 49$ \\
\hline 34 & $55 \cdot 5$ & 81 & $22 \cdot 6$ & 160 & $4 \cdot 99$ \\
\hline 35 & $54 \cdot 5$ & 82 & $22 \cdot 2$ & 165 & $4 \cdot 54$ \\
\hline 36 & $53 \cdot 4$ & 83 & $21 \cdot 8$ & 170 & $4 \cdot 12$ \\
\hline 37 & $52 \cdot 4$ & 84 & $21 \cdot 4$ & 180 & $3 \cdot 41$ \\
\hline 38 & $51 \cdot 4$ & 85 & $21 \cdot 0$ & 190 & $2 \cdot 81$ \\
\hline 39 & 50.5 & 86 & $20 \cdot 6$. & 200 & $2 \cdot 32$ \\
\hline 40 & $49 \cdot 5$ & 87 & $20 \cdot 2$ & 220 & 1.59 \\
\hline 41 & $48 \cdot 6$ & 88 & $19 \cdot \overline{8}$ & 240 & $1 \cdot 08$ \\
\hline 42 & $47 \cdot 7$ & 89 & $19 \cdot 4$ & 250 & .89 \\
\hline 43 & $46 \cdot 8$ & 90 & $19 \cdot 0$ & 275 & .55 \\
\hline 44 & $45 \cdot 9$ & 91 & 18.7 & 300 & .34 \\
\hline 45 & $45 \cdot 0$ & 92 & $18 \cdot 3$ & 325 & .21 \\
\hline 46 & $44 \cdot 1$ & 93 & 18.0 & 350 & .13 \\
\hline
\end{tabular}




\section{DECAY OF THORIUM ACTIVE DEPOSIT (LONG EXPOSURE) MEASURED BY RAYS FROM THORIUM C.}

For thorium $B, \quad T_{1}=10 \cdot 6$ hours, $\quad \lambda_{1}=06539$ (hour) ${ }^{-1}$.

For thorium $C, \quad T_{2}=1$ hour, $\quad \lambda_{2}=\cdot 6931$ (hour) $^{-1}$.

\begin{tabular}{|c|c|c|c|c|c|}
\hline Time. & Activity. & Time. & Activity. & Time. & Activity. \\
\hline $0 \mathrm{~m}$. & 100 & $7 \mathrm{~h} .45 \mathrm{~m}$. & $66 \cdot 5$ & 32 hours. & $13 \cdot 6$ \\
\hline 15 & 99.9 & 8 h. $00 \mathrm{~m}$. & $65 \cdot 4$ & 33 & $12 \cdot 7$ \\
\hline 30 & $99 \cdot 5$ & 15 & $64 \cdot 3$ & 34 & $11 \cdot 9$ \\
\hline 45 & $98 \cdot 9$ & 30 & $63 \cdot 3$ & 35 & $11 \cdot 2$ \\
\hline $1 \mathrm{~h} .00 \mathrm{~m}$. & $98 \cdot 2$ & 45 & $62 \cdot 3$ & 36 & $10 \cdot 5$ \\
\hline 15 & $97 \cdot 4$ & $9 \mathrm{~h} .00 \mathrm{~m}$. & $61 \cdot 3$ & 37 & $9 \cdot 82$ \\
\hline 30 & $96 \cdot 4$ & 15 & $60 \cdot 3$ & 38 & $9 \cdot 20$ \\
\hline 45 & $95 \cdot 4$ & 30 & $59 \cdot 3$ & 39 & 8.62 \\
\hline $2 \mathrm{~h} .00 \mathrm{~m}$. & $94 \cdot 3$ & 45 & $58 \cdot 3$ & 40 & $8 \cdot 07$ \\
\hline 15 & $93 \cdot 3$ & 10 hours. & $57 \cdot 4$ & 41 & $7 \cdot 56$ \\
\hline 30 & $91 \cdot 9$ & 11 & $53 \cdot 8$ & 42 & $7 \cdot 08$ \\
\hline 45 & $90 \cdot 7$ & 12 & $50 \cdot 4$ & 43 & $6 \cdot 64$ \\
\hline 3 h. $00 \mathrm{~m}$. & $89 \cdot 5$ & 13 & $47 \cdot 2$ & 44 & $6 \cdot 22$ \\
\hline 15 & $88 \cdot 2$ & 14 & $44 \cdot 2$ & 45 & $5 \cdot 82$ \\
\hline 30 & $86 \cdot 9$ & 15 & $41 \cdot 4$ & 46 & $5 \cdot 46$ \\
\hline 45 & $85 \cdot 6$ & 16 & $38 \cdot 8$ & 47 & $5 \cdot 11$ \\
\hline $4 \mathrm{~h} .00 \mathrm{~m}$. & $84 \cdot 4$ & 17 & $36 \cdot 3$ & 48 & $4 \cdot 79$ \\
\hline 15 & $83 \cdot 1$ & 18 & $34 \cdot 0$ & 49 & $4 \cdot 48$ \\
\hline 30 & $81 \cdot 8$ & 19 & $31 \cdot 9$ & 50 & $4 \cdot 20$ \\
\hline 45 & $80 \cdot 6$ & 20 & $29 \cdot 9$ & 51 & $3 \cdot 93$. \\
\hline $5 \mathrm{~h} .00 \mathrm{~m}$. & $79 \cdot 3$ & 21 & $28 \cdot 0$ & 52 & $3 \cdot 68$ \\
\hline 15 & $78 \cdot 1$ & 22 & $26 \cdot 2$ & 53 & $3 \cdot 45$ \\
\hline 30 & $76 \cdot 8$ & 23 & $24 \cdot 5$ & 54 & $3 \cdot 23$ \\
\hline 45 & $75 \cdot 6$ & 24 & $23 \cdot 0$ & 55 & $3 \cdot 03$ \\
\hline $6 \mathrm{~h} .00 \mathrm{~m}$. & $74 \cdot 4$ & 25 & $21 \cdot 5$ & 56 & $2 \cdot 84$ \\
\hline 15 & $73 \cdot 2$ & 26 & $20 \cdot 2$ & 57 & $2 \cdot 66$ \\
\hline 30 & $72 \cdot 1$ & 27 & $18 \cdot 9$ & 58 & $2 \cdot 49$ \\
\hline 45 & $70 \cdot 9$ & 28 & $17 \cdot 7$ & 59 & $2 \cdot 33$ \\
\hline $7 \mathrm{~h} .00 \mathrm{~m}$. & $69 \cdot 8$ & 29 & $16 \cdot 6$ & 60 & $2 \cdot 18$ \\
\hline 15 & $68 \cdot 7$ & 30 & 15.5 & 3 days. & .996 \\
\hline 30 & $67 \cdot 6$ & 31 & $14 \cdot 5$ & 4 & $\cdot 207$ \\
\hline
\end{tabular}




\section{APPENDIX III.}

For the guidance of those equipping a laboratory for radioactive measurements, it may be useful to know where radio-active materials and certain special instruments can be obtained.

Radium and actinium : Buchler \& Co., Chininfabrik, Braunschweig, Germany.

Mesothorium and radio-thorium preparations : Dr. O. Knöfler \& Co., chemische Fabrik, Plötzensee bei Berlin.

Radio-active minerals, zinc sulphide screens, etc. : F. H. Glew, Surgical Radiographer, 156 Clapham Road, London, S.W.

$\alpha, \beta$ and $\gamma$ ray electroscopes: Chas. W. Cook, University Works, Manchester.

Telemicroscopes for electroscopes: W. G. Pye, Granta Works, Cambridge.

Adjustable condenser (Gerdien), cadmium cells for electrostatic work : Messrs. Spindler \& Hoyer, Göttingen, Germany. 


\section{INDEX.}

$a$ rays, absorption of, 45 .

electroscopes, 21.

laws of probability applied to, 53 .

range of, 44, 50 .

stopping power of, 50 et seq.

variation of ionisation along path of, 46 et seq.

Actinium $A$, decay of, 101.

Actinium active deposit, 82, 83, 88; concentration of, 71 et seq.

Actinium $C$, separation of, 96, 125. decay of, 96.

Actinium emanation, decay of, 98 ; diffusion of, 103.

Actinium $X$, separation of, 124 .

$\beta$ rays, absorption of, 56 et seq., 67 .

from active deposits, 58 .

from Radium $E, 58$.

electroscope, 22.

homogeneity of, 58 .

from different thicknesses of uranium, 60.

scattering of, 61 et seq.

Bronson, constant deflection method, 13.

Cadmium cells, 8.

Calibration, of electroscope scale, 24 ; of electrometer scale, 6 .

Capacity, measurement of, 17 et seq.

Cathode rays, $\frac{e}{m}$ and velocity of, 38 .

Condensers, 16.

Curie, 111.

Decay, radio-active, 80 et seq.

Diffusion of emanations, 101 et seq.

Distortional control, 4.

Dolezalek electrometer, 1 .

Earthing key, 9.

Efficiency of recoil, 78.

Electrometer, quadrant, 1 et seq.

Electroscopes, 21 et seq.

Electroscope leaves, 25.
Emanations, decay of, 97.

Equilibrium, radio-active, 89 ; transient radio-active, 111 et seq.

Excited radiation, 68 et seq.

$\gamma$ rays, absorption of, 65 et seq.; electroscope, 22.

Half-value period, 82.

Inductional control, 5.

Insulation tests, 12.

Insulators, 26.

Ionisation currents, measurement of, 10 et seq.

Ionisation, by collision 36 ; vessels, 7 et seq.

Liquid resistances, 14.

Mesothorium, separation of, 125 .

Microscope for scintillations, 49 .

Millicurie, 111.

Natural leak, 12, 24.

Polonium, see Radium $F$.

Probability variations, $\mathbf{5 4}$.

Quartz fibre electroscope, 30.

Radio-actinium, separation of, 124.

Radium active deposit, 90 et seq., 95.

emanation, estimation of, 110 et seq. 127.

standard, 104 et seq.

standardisation of, $106 \mathrm{et} \mathrm{seq.}$

Radium $C$, separation of, 122 .

Radium $D, \quad, \quad 122$.

Radium $E, \quad$," 122.

Radium $F, \quad,, \quad 123$.

Ranges of a rays, 44, 50 .

Recoil, 75 et seq.

Recombination, 34 et seq. 
Saturation, current, 32 ; voltage for $\alpha$ and $\beta$ rays, 34 .

Scintillations, 48 et seq. ; standardisation by, 117 .

Sensitiveness of quadrant electrometer, 6.

Separation of radio-active products, 120.

Stopping power, 52.

Thorium active deposit, 83 et seq., 88.

Thorium $A$, decay of, 100.

Thorium $B$, separation of, 127 .

Thorium $C$, ,,$\quad 127$.
Thorium $X$, separation of, 125 .

Tilted electroscope, 29.

Transformation theory, 80.

Uranium oxide, films, 43 ; ionisation by different areas and thicknesses of, 42.

Uranium $X$, decay and recovery of, 94 ; separation of, 121.

Wilson, C. T. R., tilted electroscope, 29.

Wulf, quartz fibre electroscope, 30.

Zinc sulphide screens, 49 . 




(x) (1) (3)

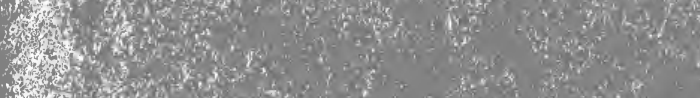

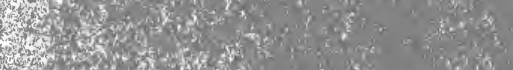
The

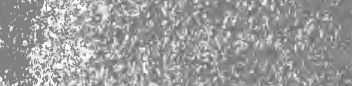

ing

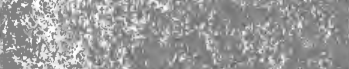

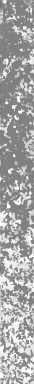
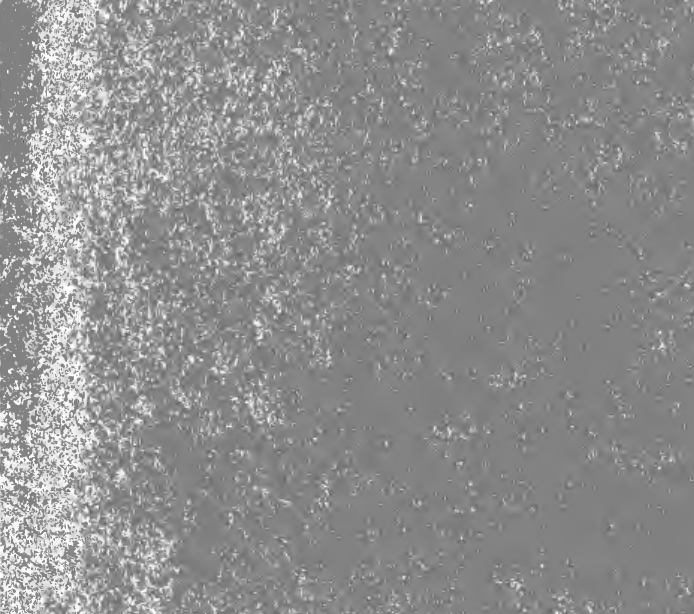

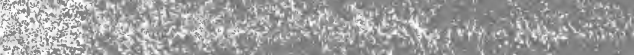




\section{UNIVERSITY OF CALIFORNIA LIBRARY BERKELEY}

Return to desk from which borrowed.

This book is DUE on the last date stamped below.

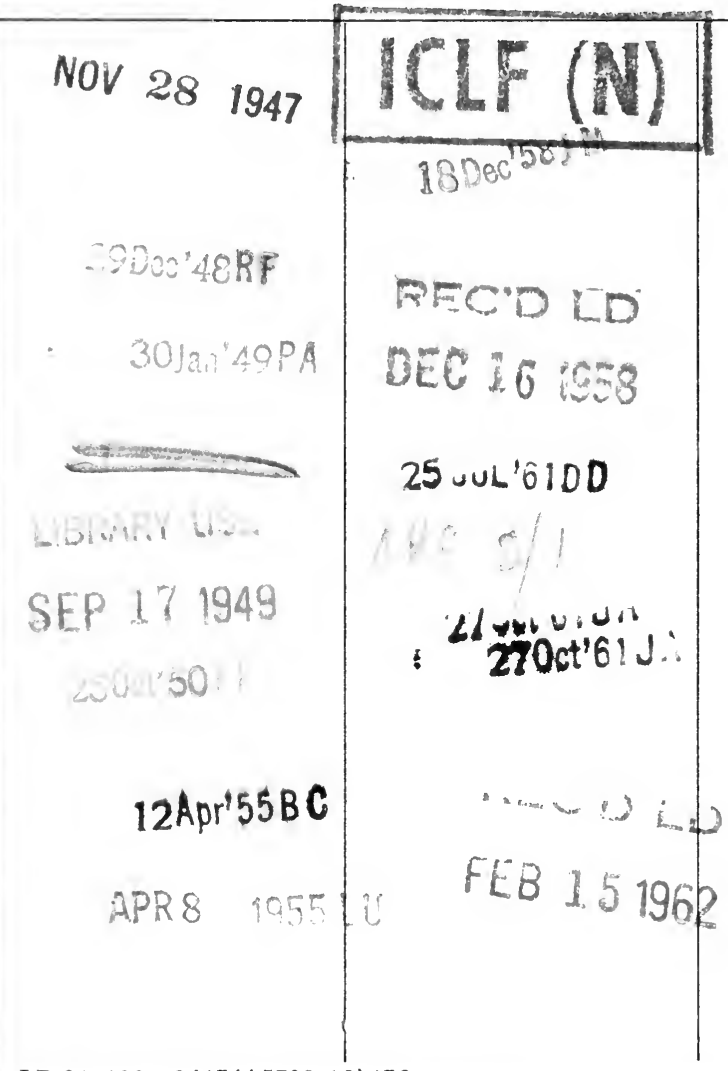


